\section{Author's Accepted Manuscript}

A review of multiscale modeling of metal-catalyzed reactions: Mechanism development for complexity and emergent behavior

M. Salciccioli, M. Stamatakis, S. Caratzoulas, D.G. Vlachos

PII:

DOI:

Reference:

To appear in: S0009-2509(11)00368-X doi:10.1016/j.ces.2011.05.050 CES 9777

Chemical Engineering Science

Received date: $\quad 4$ February 2011

Revised date: $\quad 21$ May 2011

Accepted date: $\quad 30$ May 2011

\section{MHEMICAL}

ENGINEERING SCIENCE

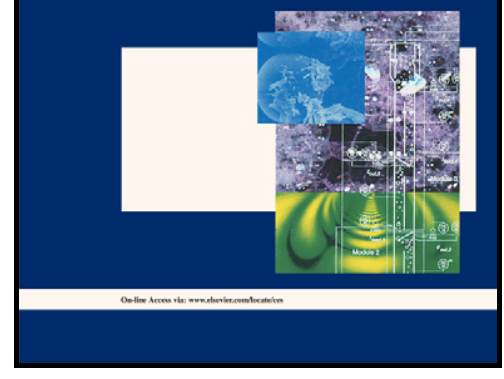

www.elsevier.com/locate/ces

Cite this article as: M. Salciccioli, M. Stamatakis, S. Caratzoulas and D.G. Vlachos, A review of multiscale modeling of metal-catalyzed reactions: Mechanism development for complexity and emergent behavior, Chemical Engineering Science, doi:10.1016/j.ces.2011.05.050

This is a PDF file of an unedited manuscript that has been accepted for publication. As a service to our customers we are providing this early version of the manuscript. The manuscript will undergo copyediting, typesetting, and review of the resulting galley proof before it is published in its final citable form. Please note that during the production process errors may be discovered which could affect the content, and all legal disclaimers that apply to the journal pertain. 


\title{
A review of multiscale modeling of metal-catalyzed reactions: Mechanism development for complexity and emergent behavior
}

\author{
M. Salciccioli, M. Stamatakis, S. Caratzoulas, and D. G. Vlachos* \\ Department of Chemical Engineering, Catalysis Center for Energy Innovation, and Center \\ for Catalytic Science and Technology, \\ University of Delaware, Newark, DE 19716-3110, USA
}

\begin{abstract}
We review and provide a perspective on multiscale modeling of catalytic reactions with emphasis on mechanism development and application to complex and emergent systems. We start with an overview of length and time scales, objectives, and challenges in first-principles modeling of reactive systems. Subsequently, we review various methods that ensure thermodynamic consistency of mean-field microkinetic models. Next, we describe estimation of reaction rate constants via quantum mechanical and statistical-mechanical methods as well as semi-empirical methods. Among the latter, we discuss the bond-order conservation method for thermochemistry and activation energy estimation. In addition, we review the newly developed group-additivity method on adsorbate/metal systems and linear free energy or Brønsted-EvansPolanyi (BEP) relations, and their parameterization using DFT calculations to generate databases of activation energies and reaction free energies. Linear scaling relations, which can enable transfer of reaction energetics among metals, are discussed. Computation-driven catalyst design is reviewed and a new platform for discovery of materials with emergent behavior is introduced. The effect of parameter uncertainty on catalyst design is discussed; it is shown that adsorbateadsorbate interactions can profoundly impact materials design. Spatiotemporal averaging of microscopic events via the kinetic Monte Carlo method for realistic reaction mechanisms is discussed as an alternative to mean-field modeling. A hierarchical multiscale modeling strategy is proposed as a means of addressing (some of) the complexity of catalytic reactions. Structurebased microkinetic modeling is next reviewed to account for nanoparticle size and shape effects and structure sensitivity of catalytic reactions. It is hypothesized that catalysts with multiple sites of comparable activity can exhibit structure sensitivity that depends strongly on operating conditions. It is shown that two descriptor models are necessary to describe the thermochemistry of adsorbates on nanoparticles. Multiscale and accelerated methods for computing free energies in solution, while accounting explicitly for solvent effects in catalytic reactions, are briefly touched upon with the acid catalyzed dehydration of fructose in water as an example. The above methods are illustrated with several reactions, such as the $\mathrm{CO}$ oxidation on $\mathrm{Au}$; the hydrogenation of ethylene and hydrogenolysis of ethane on Pt; the glycerol decomposition to
\end{abstract}


syngas on Pt-based materials; the $\mathrm{NH}_{3}$ decomposition on single metals and bimetallics; and the dehydration of fructose in water. Finally, we provide a summary and outlook.

Keywords: Catalysis; Chemical reactors; Kinetics, Simulation; Multiscale modeling; Catalyst design; microkinetic modeling

*Corresponding author (D.G. Vlachos): Email address: vlachos@udel.edu

Telephone number: 302-831-2830 


\section{Introduction}

With the interest for more energy efficient, selective and renewable chemical processes intensifying, the need to control chemical reactions at the molecular level is also rapidly increasing. Controlling reactions at the molecular level depends critically on our ability to describe the elementary reaction steps involved in a chemical transformation from reactants to products and assign a rate constant to each individual step. With this knowledge, one can predict the 'work-flow' of a chemical transformation and develop methods and catalysts to direct a chemical reaction toward a lower energy direction (easier or faster) and/or more selective pathways.

While the above goal has been the dream of the catalysis community for the past century, computational techniques have been lagging spectroscopic characterization. Also, experiments conducted on ideal catalysts (single crystals), under well-defined conditions (ultrahigh vacuum (UHV)), have not been bridged with those on supported catalysts working under realistic operating conditions. These well-known materials and pressure gaps have seriously impeded progress in heterogeneous catalysis driven by fundamentals. With the advent of abundant computational power and more accurate methods over the past decade, first-principles techniques, and specifically Density-Functional Theory (DFT), are revolutionizing our thinking on catalytic reactions. Still, our ability to describe, and eventually control, chemical transformations by first-principles modeling, at the molecular level, is hindered by multiple challenges.

In this paper, we provide a perspective on multiscale modeling for the development and simulation of catalytic reaction mechanisms. First, we provide an overview of the length and time scales in reacting systems, of the objectives of multiscale modeling, and of the challenges in first-principles modeling of chemical reactions and reactors. We also underscore the need for detailed reaction models by way of a few examples. The greater part of the review then focuses on mean-field microkinetic models and their hierarchical multiscale refinement. Emerging topics in computation-driven catalyst design and uncertainty quantification are also reviewed. Recent developments in ab initio kinetic Monte Carlo simulations are then presented, and structuredependent microkinetic models are discussed. New methods to describe catalytic chemistry in solution are outlined and an example from the homogeneous catalytic dehydrogenation of fructose to 5-hydroxylmethylfurfural is summarized. Finally, concluding remarks and an outlook are given.

\section{Overview of multiscale modeling of chemical reactions and reactors}

\subsection{Scales in reacting systems}

There are at least three scales encountered in a chemical reactor (Figure 1). At the microscopic, or electronic, length and time scales (bottom of Figure 1), adsorbate-catalyst and adsorbate-adsorbate interactions determine the potential energy surface and thus the free energy barrier and entropy of the chemical transformation. A coarse description at this scale is the free energy of transformation from reactants to the transition state (TS) and then to products. The thermal rate constant is a convenient way of coarse-graining the information from this scale, and quantum mechanical methods are ideally suited, at least in principle (see below), for this task.

Given a list of reaction events and their rate constants, adsorbates arrange themselves in spatial configurations or patterns, as a result of the collective behavior of the ensemble of all species. At this mesoscopic scale (middle of Figure 1), the collective behavior has to be averaged 
over length and time scales that are much larger than the characteristic length and time scale of the underlying pattern -- or what is known as the correlation length -- in order to compute the reaction rate. This can be achieved via non-equilibrium statistical mechanics techniques. Due to the fast vibrations of adsorbates with respect to the reaction time scales, adsorbates are typically thermally equilibrated, and reaction events can be thought of as rare events, i.e., over the time scale of a chemical reaction, the system loses its memory and can be approximated as a Markov process. The kinetic Monte Carlo (KMC) method is the most commonly used statistical technique for averaging spatiotemporal events and providing the reaction rate (Bortz et al., 1975; Chatterjee and Vlachos, 2007).

At the macroscopic (reactor) scale (top of Figure 1), there are gradients in fluid flow, concentration and temperature fields over scales that are typically much larger than the spatial inhomogeneity of the patterns of adsorbates. As a result, the reaction rate computed at the mesoscopic scale can be applied over a certain length scale (discretization size) of a chemical reactor. Due to spatial macroscopic gradients, the rate has to be evaluated at all discretization points of the macroscopic (reactor) domain.

At each scale, computation can be done with various methods whose accuracy and cost vary. As one moves from left to right of the graph at each scale, the accuracy increases at the expense of computational intensity. Thus, at each scale, one can think of a hierarchy of methods. The accuracy of these methods does not vary in a continuous fashion, i.e., each method is different. Typical methods are depicted in Figure 1. Hierarchy adds a new dimension to multiscaling: at each length and time scale, more than one model can be employed in the same simulation scheme, in order to refine the results or calculate error estimates.

\subsection{Objectives of multiscale modeling}

The early vision of multiscale modeling was rooted in the bottom-up modeling strategy for predicting the macroscopic (reactor) behavior from microscopic scale calculations (Raimondeau and Vlachos, 2002), as shown in Figure 2. This approach naturally leads to process design, control, and optimization with unprecedented accuracy. It departs significantly from the empirical process design and control strategies of the past, whereby fitting to experimental data was essential to model building.

Due to the disparity in length and time scales over which various tools apply (Figure 2), the straightforward, if not the only, way to reach macroscopic scales is by coupling models describing phenomena at different scales. Over the past fifteen years or so, several algorithms have been developed to achieve this bi-directional or two-way coupling (the branches of multiscale modeling are discussed elsewhere (Vlachos, 2005). The structural difference of models across scales (continuum vs. discrete and deterministic vs. stochastic) leads naturally to hybrid simulations, whose numerical solution poses new challenges and opportunities for multiscale mathematics. The hybrid multiscale integration algorithm (Christofides, 2001; Drews et al., 2004; Lou and Christofides, 2003; Rusli et al., 2004; Vlachos, 1997), the equation-free approach (Gear et al., 2003; Kevrekidis et al., 2004) and the heterogeneous multiscale method (E et al., 2003) are typical names and variations of coupling algorithms.

The aforementioned coupled multiscale simulations have been applied to prototype systems where the emphasis was on method development rather than on the physical systems themselves. As a result, the models were oversimplified. In catalysis, the majority of hybrid multiscale models with detailed mechanisms has tacitly ignored the coupling i.e., only one-way coupling was considered whereby the smaller scale model passed information to the next (larger) scale 
model. One of the most physically interesting, one-directional multiscale models for reactions in zeolites has recently been reported by (Hansen et al., 2010). Some examples of two-directional coupling have also been reported (Makeev et al., 2002; Raimondeau and Vlachos, 2002).

Molecular modeling, such as molecular dynamics (MD) and kinetic Monte Carlo (KMC), is often limited to short length and time (mesoscopic) scales. Over the past ten years, various coarse-graining and acceleration methods have been developed to enable molecular simulation of larger systems. The coarse-grained KMC method reviewed herein is such an example. Accelerated MD methods are briefly discussed below (Section 11). Similar to hybrid multiscale modeling, most coarse-grained KMC and to some extent MD simulations have again focused on method development. The coupling of mesoscopic and macroscopic scales of chemical reactors is covered in (Raimondeau and Vlachos, 2002; Vlachos, 1997) and will not be further discussed here. Instead our focus will be on the micro- and meso-scales where several exciting developments have taken place over the past decade.

Coupling of models across scales enables top-down modeling whereby one defines optimization targets (e.g., maximum activity and/or selectivity) and then searches for materials with suitable electronic properties. This opens up exciting opportunities for product design, rather than just process design (Vlachos et al., 2006). In Section 6 we discuss how this concept is put to use for computation-driven catalyst design, especially of materials exhibiting emergent behavior.

\subsection{Challenges in multiscale modeling of catalytic systems}

Multiscale modeling of reactions and reactors imposes multiple challenges, as shown in Figure 2 and Figure 3. The first challenge in describing heterogeneous catalytic chemistry via first principles is that phenomena involving chemical reactions and reactors are multiscale in nature (Raimondeau and Vlachos, 2002; Vlachos, 2005). Individual reaction events take place on specific sites of a catalyst, at the sub-nanometer length scale and over picosecond to nanosecond time scales. Yet, the macroscopic behavior is determined by the collective behavior of reaction events (ensemble average), which emerges over length scales ranging from nanometers to millimeters and evolves over time scales from picoseconds to milliseconds or longer. Internal and external transport processes further complicate the observed behavior by creating an intimate coupling between microscopic events and macroscopic reactor processes. As a result, first principles methods alone cannot predict common experimental observables (e.g., reaction rates, selectivity, coverage of intermediates, etc.).

A second and often overlooked challenge is the many-body nature of a catalytic reaction. Unlike gas-phase reactions, where reaction events are a result of a bimolecular collision and associated energy transfer, the local environment of co-reactants in a surface reaction can have a profound effect on the speed of a transformation (catalyst activity) and the specific pathway it follows (catalyst selectivity). The local environment entails co-adsorbates or the specific arrangement of catalyst atoms in close proximity to the reactant pair. As a result, the parameterization of a rate constant with a simple Arrhenius form with constant parameters (preexponential, activation energy), as in gas-phase reactions, is generally inadequate for surface reactions. The functional dependence of kinetic rate constant parameters on coverage (e.g., linear dependence of activation energy on coverage) is a simplistic (mean-field) way of dealing with these many body effects that fundamentally does not address the challenge itself.

Catalytic reactions exhibit intrinsic heterogeneity in both adsorbate distribution and catalyst sites. Adsorbate inhomogeneity is the result of intermolecular forces and/or chemical reactions 
and is manifested as spatiotemporal patterns of adsorbates with a characteristic length that depends on the chemical reaction and operating conditions. The $\mathrm{p} 2 \mathrm{x} 2$ adsorbate structure or the spatiotemporal patterns in CO oxidation are such examples (Ertl, 1991). As a result, the adsorbates' distribution cannot be assumed uniform, even at a length scale that is small (pellet size or even smaller) compared to that of a chemical reactor, as typically done in the so called mean-field approximation. Catalyst inhomogeneity can be manifested in multiple ways (Figure 3). One example entails nanoparticles and clusters, consisting of distinctly different types of catalyst sites. Another example is a particle size distribution due to competition of nucleation and growth during synthesis. Such adsorbate and catalyst non-uniformities can span length scales from nanometer to millimeter and control macroscopic reaction rates at the pellet scale.

Another challenge in modeling catalytic reactions arises from the multiphase nature of chemical reactions in condensed matter. Reactions carried out in solvents, such as aqueous reforming of biomass over noble metals or dehydration of fructose to hydroxyl methyl furfural in $\mathrm{HCl}$, are strongly affected by the solvent. For example, the solvent can participate in the TS, e.g., via hydrogen bonding or even in the reaction itself, e.g., via solvent-assisted proton transfer, and affect the reaction mechanism or the reaction rate. As a result, free energies rather than energies in the presence of explicit solvent are necessary for computing reaction rate constants. This task requires conformation sampling and averaging using statistical mechanics. MD is an ideally suited method for this task, but large free energy barriers render classical and $a b$ initio MD methods impractical. Similarly, the catalyst support can often strongly affect reactivity by modifying the catalyst nanoparticle size and shape, via creating interfacial support/metal sites, and by electronic coupling, such as electron exchange with the metal.

Finally, it is commonplace to assume that the catalyst is static over the time scale of a chemical reaction. In reality, the catalyst is dynamic in response to its local environment, such as the gases and adsorbates present, the temperature, the pressure, etc. Catalyst dynamics, such as reconstruction and isomerization between various conformations, maybe more common than we currently think, but their effect on the kinetics of catalytic reactions is poorly understood.

\section{Closed-form empirical kinetic models}

Here we give a brief overview of the types of empirical reaction models discussed in (Raimondeau and Vlachos, 2002) to underscore the need for detailed surface reaction mechanisms. The connection between multiscaling and detailed reaction mechanisms becomes transparent later.

\subsection{Power-law kinetics}

In early days, the power-law functional form was the most common type of rate expression.

$$
\mathrm{r}=\mathrm{k}_{\mathrm{eff}} \mathrm{C}_{\mathrm{A}}^{\mathrm{a}} \mathrm{C}_{\mathrm{B}}^{\mathrm{b}} ; \mathrm{k}_{\text {eff }}=\mathrm{A}_{\text {eff }} \mathrm{e}^{-\mathrm{E}_{\text {eff }} / \mathrm{RT}}
$$

Eq. 1 estimates the rate using an effective rate constant, reaction orders, and activation energy. Despite its fundamental limitations and lack of predictive power, power-law rate expressions are still commonly used in reactor and process design since they involve a small number of parameters that can easily be regressed to a limited number of experimental data. Given its limited value, this type of model is not further discussed here. 


\subsection{Langmuir-Hinshelwood-Hougen-Watson (LHHW) models}

A LHHW model starts with a detailed surface reaction mechanism. Subsequently, a priori assumptions about fast and slow processes are made. For example, it is commonly assumed that adsorption-desorption processes of reactants and products are in partial equilibrium (PE) and one of the surface reaction steps is the rate-determining step (RDS). Upon development of a closed form rate expression, one examines whether the rate expression provides a qualitative description of the experimental data. If not, a different set of assumptions is made and a new rate expression is developed (an iterative process). If the model appears to qualitatively capture experimental data, the rate and equilibrium constants of the LHHW expression are fitted using (a limited number of) experimental data from a single kind of experiment, e.g., ignition or conversion and selectivity data.

Due to their empirical nature, multiple rate expressions can describe the same data with similar statistics, i.e., the rate expressions are not unique (rate expression multiplicity (Prasad et al., 2009). In addition, multiple parameter values, which describe experimental data reasonably well, may exist for the same rate expression (rate constant multiplicity). Even when a rate expression reproduces the data reasonably well, it may describe the wrong physics (assumptions are incorrect) or the parameters may be physically unrealistic. Consequently, the model may be unable to predict underlying properties such as the most abundant surface intermediate (MASI), which controls blocking of catalyst sites, or quantities such as the effective reaction orders. Finally, LHHW rate expressions, even if correct, are typically limited to one regime of operating conditions where the rate changes monotonically with respect to a parameter. They cannot describe changes in the RDS with varying operating conditions, often manifested with maxima or minima in the response (e.g., activity).

In order to illustrate the aforementioned limitations, we provide some examples. In the steam reforming of methane over Ni catalyst, the well-known Xu and Froment model (Xu and Froment, 1989), shown in Table 1, is used. The model indicates a complex dependence on concentration of reactant species. In contrast, experiments conducted under kinetically relevant, well-controlled conditions indicate that the overall reaction is fairly simple first-order in methane and zero-order in steam (Wei and Iglesia, 2004). In addition, the reaction rate and its dependence on pressure developed by Numaguchi and Kikuchi (Numaguchi and Kikuchi, 1988) (also shown in Table 1) differ significantly from those of $\mathrm{Xu}$ and Froment, seriously questioning the validity of these empirical rate expressions.

As a last example, a LHHW model was recently developed for the partial oxidation of methane over a $\mathrm{Rh} / \mathrm{a}-\mathrm{Al}_{2} \mathrm{O}_{3}$ catalyst (Tavazzi et al., 2006). Even though the model describes data fairly well over a range of operating conditions, the parameters are not physically relevant, as shown in Table 2. These examples underscore the fact that although LHHW models are capable of fitting experimental data reasonably well, the fundamental mechanisms may still be elusive and the physical significance of parameters can be questionable.

\section{Mean-field microkinetic modeling}

Given the limitations of LHHW models, it is becoming increasingly clear that detailed surface reaction mechanisms need to be developed. The term microkinetic model implies the use of a detailed reaction mechanism describing elementary-like processes occurring on a catalyst. Detailed reaction mechanisms have been used in gas-phase reactors, such as flames and chemical vapor deposition reactors, for decades. Detailed catalytic reaction models have been reported as early as in 1986 and a few papers have appeared thereafter (Hickman and Schmidt, 1993; Oh et 
al., 1986). The microkinetic modeling framework was formalized by the book of Dumesic and co-workers (Dumesic et al., 1993). In these models, one lays down all relevant elementary steps and solves the mathematical problem without any assumptions about a RDS, PE of reactions, quasi-steady state (QSS) assumption of intermediates, or a specific MASI.

Unlike power-law expressions, which are regressed to a specific range of experimental conditions, detailed kinetic models are predictive over an extended range of experimental conditions (Prasad et al., 2009). Ultimately, this sequence of elementary reactions gives fundamental insights into surface intermediates, reaction rates, reaction rate sensitivity to specific model parameters, and can be used as a predictive tool for reactor optimization. General chemical kinetic codes, such as CHEMKIN (Coltrin et al., 1991; Kee et al., 1991a), used for detailed reaction mechanism studies, enable a straightforward reaction mechanism implementation.

Such implementations have the great advantages of being widely applicable and modular. Conventionally, a mechanism containing elementary steps can be applied to various chemistries that contain similar reactant, product and reactive intermediates (Gokhale et al., 2004). For example, the water-gas shift reaction, the $\mathrm{CO}$ oxidation, and the selective oxidation of $\mathrm{CO}$ share several elementary steps. In addition, one can build mechanisms of larger species from those of smaller ones, making mechanism-building easier. For example, the steam reforming of ethane encompasses also the elementary steps of methane steam reforming and of the water-gas shift reaction.

In the first generation of microkinetic models (up to the late 1990s), the rate constants were fitted to experimental data. The lack of methods for estimating a large number of parameters limited the use of microkinetic modeling and confidence in their predictive ability abated. Over the past decade (2000s), semi-empirical methods offered an inexpensive and fairly accurate approach for estimating activation energies of simple adsorbates participating in small reaction networks. This approach worked well for small molecules, such as $\mathrm{CO}, \mathrm{NH}_{3}$ and $\mathrm{CH}_{4}$. The abundant computer power available nowadays has made possible the estimation of rate constants through first-principles methods, and in particular density functional theory (DFT). A summary of estimation methods is shown at the bottom of Figure 1. Table 3 depicts examples of recently published microkinetic models developed using different estimation methods, along with their applications.

Mean-field models are based on the assumption of a uniform distribution of adsorbate and catalyst sites. They couple mechanistic energetics with reaction rates and species concentrations, and thus, they link fundamental quantum mechanical and statistical mechanical calculations to reactor scale phenomena.

A reaction is represented as $\sum_{k} v_{k} S_{k}=0$, where $v_{k}$ is the stoichiometric coefficient of species $S_{k}$. The reaction rate of an elementary, irreversible reaction follows mass action kinetics as follows:

$$
r_{i}=k_{i} \prod_{j} C_{j}=A_{i} \exp \left(\frac{-\Delta E_{i}}{k_{B} T}\right) \prod_{j} C_{j}
$$

Here $k_{i}$ is the forward rate constant reaction $i, A_{i}$ is the pre-exponential factor, $\Delta E_{i}$ is the activation energy, $k_{B}$ is the Boltzmann constant, $T$ is the absolute temperature, and $C_{j}$ is the reactant concentration. This parameterization of surface reaction rates follows the same logic as that of bimolecular gas-phase reactions, i.e., the rate constant is taken to be independent of the 
local environment in which a reaction takes place. Due to the many-body nature of catalytic reactions, this is a major conceptual limitation. In this section, we follow this common assumption and describe corrections in later sections.

Kinetic models used to describe wide temperature ranges often employ the modified Arrhenius equation. The pre-exponential factor is written as, e.g., (Maestri et al., 2009; Maestri et al., 2008; Mhadeshwar and Vlachos, 2005c; Mhadeshwar and Vlachos, 2007; Salciccioli et al., 2011):

$$
A_{i}=A_{i}^{\prime}\left(\frac{T}{T_{0}}\right)^{\beta} \text { or } A_{i}=A_{i}^{\prime}(T)^{\beta}
$$

This modified form is used in the thermodynamic (Kee et al., 1991a) and kinetic modeling tool CHEMKIN (Coltrin et al., 1991; Kee et al., 1991a) and provides flexibility for fitting $\beta$ of sensitive reactions to experimental data or for temperature dependent pre-exponential factors calculated from transition state theory (TST). As alluded to above, TST relates rate constants with the Gibbs free energy of reactant, product and transition states.

$$
\begin{gathered}
\mathrm{k}_{\mathrm{i}}=\frac{\mathrm{k}_{\mathrm{B}} \mathrm{T}}{\mathrm{h}} \exp \left(\frac{-\Delta \mathrm{G}_{\mathrm{i}}^{\dagger}}{\mathrm{k}_{\mathrm{B}}^{\mathrm{T}}}\right)=\frac{\mathrm{k}_{\mathrm{B}} \mathrm{T}}{\mathrm{h}} \exp \left(\frac{\Delta \mathrm{S}_{\mathrm{i}}^{\ddagger}}{\mathrm{k}_{\mathrm{B}}}\right) \exp \left(\frac{-\Delta \mathrm{H}_{\mathrm{i}}^{\dagger}}{\mathrm{k}_{\mathrm{B}} \mathrm{T}}\right) \\
\mathrm{K}_{\mathrm{i}}=\exp \left(\frac{-\Delta \mathrm{G}_{\mathrm{i}}}{\mathrm{k}_{\mathrm{B}} \mathrm{T}}\right)=\exp \left(\frac{\Delta \mathrm{S}_{\mathrm{i}}}{\mathrm{k}_{\mathrm{B}}}\right) \exp \left(\frac{-\Delta \mathrm{H}_{\mathrm{i}}}{\mathrm{k}_{\mathrm{B}} \mathrm{T}}\right)
\end{gathered}
$$

Eq. 4 describes the irreversible elementary reaction rate constant of reaction $\mathrm{i}\left(\mathrm{k}_{\mathrm{i}}\right)$ as a function of the change in Gibbs free energy from reactant to transition state $\left(\Delta \mathrm{G}_{\mathrm{i}}^{\dagger}\right)$, and further as a function of change in entropy $\left(\Delta \mathrm{S}_{\mathrm{i}}{ }^{\ddagger}\right)$ and enthalpy $\left(\Delta \mathrm{H}_{\mathrm{i}}^{\ddagger}\right)$ from reactant to transition state. An additional factor consisting of the Boltzmann constant, absolute temperature and Planck's constant $\left(\mathrm{k}_{\mathrm{B}} \mathrm{T} / \mathrm{h}\right)$ approximates the contribution from the frequency associated with the negative curvature of the potential energy surface in the direction of the reaction coordinate (Houston, 2001). Figure 4a shows a schematic of thermochemical property evolution along the reaction coordinate of an elementary reaction. The reaction coordinate is the arc-length along the path of minimum potential energy from reactant to product. Eq. 5 gives the equilibrium constant as a function of free energy of reaction (first equality) and entropy and enthalpy of reaction (second equality).

\subsection{Thermodynamic consistency in microkinetic modeling}

An important aspect of microkinetic model development that is often overlooked when combining the kinetic and thermodynamic parameters of individual species and elementary reactions into a mechanism is thermodynamic consistency. The importance of ensuring thermodynamic consistency in kinetic mechanism development cannot be overstated. First, the underlying equilibrium constants of reaction mechanisms are directly tied to thermodynamic quantities. If these equilibrium constants are incorrect, predictions pertaining to equilibrium limited processes will be inaccurate. Secondly, under non-isothermal conditions inconsistencies between the kinetic parameters and thermodynamic properties result in incorrect solutions of the energy balance and thus of temperatures and compositions (Mhadeshwar et al., 2003).

The forward (f) and backward (b) kinetic parameters are related to the equilibrium constant as follows: 


$$
\mathrm{K}_{\mathrm{i}}=\frac{\mathrm{k}_{\mathrm{i}, \mathrm{f}}}{\mathrm{k}_{\mathrm{i}, \mathrm{b}}}
$$

Thermodynamic constraints were described comprehensively by Dumesic and co-workers in four equations (Dumesic et al., 1993). First, thermodynamic consistency of individual elementary reactions implies that:

$$
\begin{gathered}
\Delta E_{i, b}=\Delta E_{i, f}-\Delta H_{i} \\
A_{i, b}=A_{i, f} \exp \left(\frac{\Delta G_{i}-\Delta H_{i}}{R T}\right)
\end{gathered}
$$

Here, $\Delta \mathrm{E}_{\mathrm{i}, \mathrm{b}}$ is the backward activation energy, $\Delta \mathrm{E}_{\mathrm{i}, \mathrm{f}}$ is the forward activation energy, and $\Delta \mathrm{H}_{\mathrm{i}}$ is the standard enthalpy change of reaction. $A_{i, b}$ is the reverse pre-exponential factor, $A_{i, f}$ is the forward pre-exponential factor, $\Delta \mathrm{G}_{\mathrm{i}}$ is the change in standard Gibbs free energy of reaction, $T$ is the absolute temperature, and $\mathrm{R}$ is the ideal gas constant (Dumesic et al., 1993). The term in parenthesis is the equivalent of the standard change in entropy of reaction $\left(\Delta \mathrm{S}_{\mathrm{i}}\right)$ divided by $\mathrm{R}$.

A net reaction, starting from gaseous reactants and ending with gaseous products (Figure 4b), is often invoked that can be expressed as a linear combination of several reactions. The following equations then apply:

$$
\begin{aligned}
& \sum_{i} \sigma_{i}\left(\Delta E_{i, f}\right)-\sum_{i} \sigma_{i}\left(\Delta E_{i, b}\right)=\Delta H_{\text {net }} \\
& \prod_{i}\left(\frac{A_{i, f}}{A_{i, b}}\right)^{\sigma_{i}}=\exp \left(\frac{\Delta G_{n e t}-\Delta H_{n e t}}{R T}\right)
\end{aligned}
$$

In Eqs. 9 and 10 (Dumesic et al., 1993), the subscript "net" denotes the change in the thermodynamic state properties from net reactants to net products. The factor of $\sigma_{\mathrm{i}}$ denotes the coefficient of elementary reaction $i$ in the linear combination comprising the desired net reaction. If one further considers a gas-phase reaction having the same stoichiometry as the net reaction just noted, a thermodynamic cycle is formed, along which free energies and entropies are conserved (Hess's law).

Figure $4 \mathrm{~b}$ illustrates the interplay between the gas and surface-phase thermochemical properties of gas and surface intermediates and transition states for the simplified $A \leftrightarrow B \leftrightarrow C$ mechanism. $\zeta$ denotes any thermochemical property involved in rate and equilibrium constant evaluation $(\mathrm{H}, \mathrm{S}, \mathrm{G})$. In Figure $4 \mathrm{~b}$ property changes associated with the elementary gas-phase reactions $\mathrm{A} \leftrightarrow \mathrm{B}$ and $\mathrm{B} \leftrightarrow \mathrm{C}$ are $\Delta \zeta_{1 \text {,gas }}$ and $\Delta \zeta_{2, \text { gas }}$, respectively, and for the overall gas-phase reaction $\mathrm{A} \leftrightarrow \mathrm{C}$ is $\Delta \zeta_{3 \text {,gas }}=\Delta \zeta_{1 \text {,gas }}+\Delta \zeta_{2 \text {,gas }}$. If not experimentally known, these gas-phase properties are often calculated from high level ab initio calculations (accurate within a couple of $\mathrm{kcal} / \mathrm{mol}$ ) described elsewhere (Baboul et al., 1999; Curtiss et al., 2007; Raghavachari et al., 1997a; Raghavachari et al., 1997b; Wang and Brezinsky, 1998). While these methods have recently been shown to be accurate for energies of transition metal-containing molecules (Mayhall et al., 2009), the expense associated with calculating properties for adsorbates on several metal atom clusters for entire reaction mechanisms is unreasonable. For this reason, generalized gradient approximation (GGA) functionals are commonly employed for calculating structures and properties of adsorbate-metal configurations. This type of method strikes a reasonable compromise between accuracy and cost, and satisfies the uniform density limit (which is an important constraint for predicting bulk metal properties) (Sholl and Steckel, 2009). It is also important to note that distinct GGA functionals are developed for specific calculations 
and for specific types of molecules. Venturing outside of these limitations will result in questionable results (Sholl and Steckel, 2009). Additionally, when considering the most common computational methods methods used (which balance accuracy and expense) for surface-phase vs gas-phase thermochemical properties, the accuracy of surface thermochemistry is lower than that of the gas-phase thermochemistry. So while Eqs. (6-10) that govern the thermodynamic constraints of the kinetic parameters in microkinetic models (shown in Figure $4 b)$ are exact, the difficulty arises in their implementation.

\section{Examples of model thermodynamic consistency and violation}

To illustrate this difficulty in implementation, parameters of a subset of the water-gas shift chemistry are shown in Table 4 (taken from the thermodynamically consistent model of (Mhadeshwar and Vlachos, 2007)). Specifically, this is the reaction pathway from $\mathrm{CO}$ and $\mathrm{H}_{2} \mathrm{O}$, through the carboxyl intermediate $(\mathrm{COOH})$, to form $\mathrm{CO}_{2}$ and $\mathrm{H}_{2}$. The reaction is slightly exothermic, and thus thermodynamically limited at higher temperatures. This makes the thermodynamic consistency of water-gas shift mechanism extremely important in terms of being able to predict equilibrium conversion accurately. Using the subset of reactions below, the resulting equilibrium constant of the water-gas shift reaction $\left(\mathrm{CO}+\mathrm{H}_{2} \mathrm{O} \leftrightarrow \mathrm{CO}_{2}+\mathrm{H}_{2} \mathrm{O}\right)$ is calculated from the linear combination of all seven reactions (the product $K_{1} K_{2} K_{3} K_{4} K_{5} K_{6} K_{7}$ with $\mathrm{K}_{\mathrm{i}}$ calculated from Eqs. 3, 4 and 6). At $300 \mathrm{~K}, \mathrm{~K}_{\text {WGS }}$ calculated from this catalytic cycle is 109,100. This value agrees reasonably with that obtained from NIST gas-phase thermochemical properties (Burgess, 2009) of 93,500. The slight discrepancy arises from the implementation of the thermodynamic constraints. Since irreversible reactions were defined (one forward and one backward for each elementary reaction), exact thermodynamic consistency at all temperatures is difficult to ensure; relative tolerances result in errors that are amplified due to the exponential relationship between Gibbs free energy and the equilibrium constant (Eq. 5). This results in a small $(\sim 15 \%)$ error in $\mathrm{K}_{\text {WGS }}$ at $300 \mathrm{~K}$ with respect to the NIST value.

As an example of violation of the thermodynamic constraints, consider altering one reaction's activation energy by $2 \mathrm{kcal} / \mathrm{mol}$ in order to better describe experimental data in a kinetically limited regime. The adjustment of a single activation energy of irreversible reactions of a thermodynamically consistent mechanism leads to thermodynamic violations. For example, by increasing the $\Delta \mathrm{E}_{\mathrm{i}}$ of the forward reaction $4\left(\mathrm{CO}^{*}+\mathrm{OH}^{*} \rightarrow \mathrm{COOH}^{*+*}\right)$ from 19.1 to 21.1 $\mathrm{kcal} / \mathrm{mol}$, the $\mathrm{K}_{\text {WGS }}$ calculated from the catalytic cycle is 3,800 . This value is dramatically different from that calculated from the NIST database and leads to predicting concentration with considerable error in equilibrium-limited regimes. The disturbance of equilibrium constants from their correct values (as seen in the example) can be avoided if kinetic parameters are adjusted in a pair-wise fashion for irreversibly defined reactions.

Next, we use the same WGS mechanism to illustrate the importance of constraining surface DFT calculations (less accurate) to gas-phase thermochemistry (more accurate) in kinetic model development. As an example, reaction energies from DFT calculations (Grabow et al., 2008) are shown in the last column of Table 4. Using these energies as the Gibbs free energy (neglecting zero-point energy (ZPE) corrections, temperature corrections at $300 \mathrm{~K}$ and the PV correction, as often done in the literature), the $\mathrm{K}_{\mathrm{WGS}}$ at $300 \mathrm{~K}$ is $1.1 \times 10^{15}$, several orders of magnitude larger than the NIST calculated equilibrium constant given above. This error in $\mathrm{K}_{\text {WGS }}$ corresponds to a difference in $\Delta \mathrm{G}_{\mathrm{rxn}}$ of $\sim 13 \mathrm{kcal} / \mathrm{mol}$. Use of these uncorrected DFT energies in a microkinetic model would, in this case, over-predict the conversion for equilibrium-limited conditions. While 
simplistic, this example demonstrates the necessity of enforcing Eqs. 6-10 in kinetic mechanism development, even when using self-consistent DFT calculations.

Thermodynamic consistency through the adjustment of surface reaction properties

Thermodynamic consistency is straightforward to enforce when a single method is employed, but less so when a multitude of estimation-methods are employed. For first-principle or semiempirical-based microkinetic models, the major difficulty with thermodynamic consistency is merging gas-phase properties with surface properties. The former are computed using ab initio, high level theory and are considered to be 'exact' (with an error of $1-2 \mathrm{kcal} / \mathrm{mol}$ ). Additionally, constraining a mechanism to gas-phase properties avoids inconsistencies when combining multiple models that share gas-phase species. For example, one can build an ethane oxidation mechanism into a propane oxidation mechanism, as the $\mathrm{C} 2$ and $\mathrm{C} 1$ chemistry are submechanisms of the C3 mechanism. There are two major schools of thought on how to constrain the surface mechanism to gas-phase properties. The first centers on using adsorption properties as a basis set. This methodology is useful when integrating semi-empirical thermochemical property techniques or not having the resources to calculate partition functions for all surface intermediates and transition states.

$$
\zeta_{\mathrm{i}, \text { surf }}\left(\mathrm{T}, \theta_{\mathrm{j}}\right)=\zeta_{\mathrm{i}, \mathrm{gas}}(\mathrm{T})+\Delta \zeta_{\mathrm{ads}, \mathrm{i}}\left(\mathrm{T}, \theta_{\mathrm{j}}\right)
$$

In Eq. 11, the surface species properties $\left(\zeta_{\mathrm{i}, \text { surf }}\right)$ are defined through the corresponding gasphase species and the change in state property associated with adsorption. This quantity can also be a function of temperature or surface coverages. Upon defining the thermophysical properties

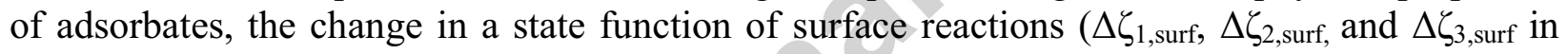
Figure $4 \mathrm{~b})$ can easily be related to those of the gas-phase reactions $\left(\Delta \zeta_{1 \text {,gas }}, \Delta \zeta_{2 \text {,gas, }}\right.$, and $\Delta \zeta_{3 \text {,gas }}$ in Figure $4 \mathrm{~b})$ and the adsorption properties of the intermediates $\left(\Delta \zeta_{\mathrm{A}, \text { ads }}, \Delta \zeta_{\mathrm{B} \text {,ads, }}\right.$, and $\Delta \zeta_{\mathrm{C} \text {,ads }}$, as shown in Figure 4b).

In 2003, a methodology for ensuring thermodynamic consistency was presented by Mhadeshwar et al. which involved constraining kinetic parameters by the use of a linearly independent basis set of elementary reactions (Mhadeshwar et al., 2003). This approach essentially connects the thermochemical properties of surface species to the thermochemical properties of gas species through the forward and reverse kinetic parameters of the linearly independent reactions. It was suggested that the difference between originally calculated reaction properties and newly defined reaction properties be divided equally between the forward and reverse rate constant (Mhadeshwar et al., 2003) (this is not a unique way). Building on this notion, methods were later developed to divide this error between the forward and reverse rate constants using as criterion the proximity of the transition state to reactants or products. In these cases, a proximity factor (Grabow et al., 2008) or a modified bond index (used in association with unity bond index-quadratic exponential potential (UBI-QEP) based models, see below) (Maestri and Reuter, 2011; Maestri et al., 2009; Mhadeshwar and Vlachos, 2007) is defined and is related to the location of the transition state along the reaction coordinate.

$$
\Delta E_{i}=\Delta E_{i, D F T}+\omega\left(\Delta H_{i, s u r f}\left(T, \theta_{i}\right)-\Delta E_{i, r x n, D F T}\right)
$$

Eq. 12, taken from a recent water-gas shift microkinetic model (Grabow et al., 2008), describes this implementation. The activation energy used in the microkinetic model $\left(\Delta \mathrm{E}_{\mathrm{i}}\right)$ is the DFT calculated activation energy, plus the proximity factor $(\omega)$ multiplied by the difference between the defined heat of surface reaction $\left(\Delta \mathrm{H}_{\mathrm{i} \text {,surf }}\right)$ through the gas-phase thermodynamic loop and the 
energy of reaction from the DFT calculation $\left(\Delta \mathrm{E}_{\mathrm{i}, \mathrm{DFT}}\right)$. A similar equation can be used to adjust the entropic terms.

$$
A_{i}=A_{i, T S T} \exp \left[\omega\left(\frac{\Delta S_{i, \text { surf }}(T)}{R}-\frac{\Delta S_{i, T S T}}{R}\right)\right]
$$

In Eq. 13, the pre-exponential factor used is the TST calculated pre-exponential factor $\left(\mathrm{A}_{\mathrm{i}, \mathrm{TST}}\right)$ multiplied by a correction term that includes the proximity factor and the difference in entropy of reaction of the original system and the one constrained to the gas-phase species. One advantage of this type of scheme is the convenient implementation into commercial kinetic modeling software. Thermochemical properties can be stored in a NASA polynomial database (Eqs. 14-16), as in CHEMKIN (Kee et al., 1991a). Then, one can easily adjust the coefficients to convert the properties of a gas-phase species into those of its corresponding surface-phase intermediate.

$$
\begin{gathered}
\frac{C_{p}}{R}=a_{1}+a_{2} T+a_{3} T^{2}+a_{4} T^{3}+a_{5} T^{4} \\
\frac{H}{R T}=a_{1}+a_{2} \frac{T}{2}+a_{3} \frac{T^{2}}{3}+a_{4} \frac{T^{3}}{4}+a_{5} \frac{T^{4}}{5}+\frac{a_{6}}{T} \\
\frac{\mathrm{S}}{R}=a_{1} \ln T+a_{2} T+\frac{a_{3}}{2} T^{2}+\frac{a_{4}}{3} T^{3}+\frac{a_{5}}{4} T^{4}+a_{7}
\end{gathered}
$$

Coefficients $\mathrm{a}_{1}-\mathrm{a}_{7}$ are those of the gaseous species, often available in gas-phase thermodynamic databases, such as that of NIST. Eqs. 17 and 18 show the adjustments needed to define the properties of surface species i (index dropped above for simplicity), based on the corresponding gas species and the change in entropy and enthalpy upon adsorption. It should be noted that to account for coverage, and to some extent temperature, effects, one would need to extend CHEMKIN (Kee et al., 1991b) so the last coefficients are temperature and coverage dependent

$$
\begin{gathered}
\mathrm{a}_{6, \text { surf }}=\mathrm{a}_{6, \text { gas }}+\frac{\Delta \mathrm{H}_{\mathrm{i} \text {,ads }}\left(\mathrm{T}, \theta_{\mathrm{j}}\right)}{\mathrm{R}} \\
\mathrm{a}_{7, \text { surf }}=\mathrm{a}_{7, \text { gas }}+\frac{\Delta \mathrm{S}_{\mathrm{i}, \text { ads }}\left(\mathrm{T}, \theta_{\mathrm{j}}\right)}{\mathrm{R}}
\end{gathered}
$$

Here $\Delta \mathrm{H}_{\mathrm{i} \text {,ads }}$ and $\Delta \mathrm{S}_{\mathrm{i} \text {,ads }}$ are the heat and entropy of adsorption of species I, which are generally functions of temperature $\mathrm{T}$ and coverage of all species, $\theta_{\mathrm{j}}$. This method is easily implemented. However, one drawback is the adjustment of competing surface reaction activation energies, which will be discussed in the following example. While not necessarily accurate in an absolute sense, one advantage of periodic DFT calculations for energetic profiles is the high relative accuracy (Blaylock et al., 2009; Sholl and Steckel, 2009). The use of the proximity factor for adjustment of reaction properties can cancel out this benefit from using DFT energies.

As an example, Eq. 12 is used to adjust two competing reactions for ethanol decomposition on $\mathrm{Pd}(111): \mathrm{CH}_{3} \mathrm{CH}_{2} \mathrm{OH}^{*} \rightarrow \mathrm{CH}_{3} \mathrm{CHOH}^{*}+\mathrm{H}^{*}$ and $\mathrm{CH}_{3} \mathrm{CH}_{2} \mathrm{OH}^{*} \rightarrow \mathrm{CH}_{2} \mathrm{CH}_{2} \mathrm{OH}^{*}+\mathrm{H}^{*}$

$$
\Delta H_{i, \text { surf }}=\sum_{j} v_{j}\left(H_{f, 298}^{j, \text { gas }}+Q_{j}\right)
$$

Based on Eq. 19, the heat of surface reaction $\mathrm{i}$ in Eq. 12 is defined based on gas-phase heats of formation $\left(\mathrm{H}_{\mathrm{f}, 298}^{\mathrm{j}, \text { gas }}\right)$, the heat of chemisorption $\left(\mathrm{Q}_{\mathrm{j}}\right)$ and the stoichiometric coefficient $\left(v_{\mathrm{j}}\right)$ for each reaction intermediate j. Taking DFT data from Li et al. (Li et al., 2009) and gas-phase 
thermochemical data from Dyke et al. (Dyke et al., 1997), Table 5 shows the application of Eq. 12 to the first dehydrogenation reactions for ethanol on $\operatorname{Pd}(111)$. The DFT barriers for decomposition to $\mathrm{CH}_{3} \mathrm{CHOH}$ or $\mathrm{CH}_{2} \mathrm{CH}_{2} \mathrm{OH}$ are similar (21.3 vs. $\left.21.9 \mathrm{kcal} / \mathrm{mol}\right)$, which would imply competition between reactions with comparable rates. Once the thermodynamic correction (Eq. 12) is implemented, the barrier for $\mathrm{CH}_{2} \mathrm{CH}_{2} \mathrm{OH}$ formation becomes $\sim 5 \mathrm{kcal} / \mathrm{mol}$ lower than the barrier for $\mathrm{CH}_{3} \mathrm{CHOH}$ formation. In this case, the enforcement of thermodynamic consistency through adsorption properties makes the relative magnitude of rate constants depart from that of the DFT calculations.

Thermodynamic consistency through the adjustment of adsorption properties

The above problem is avoided if we use another thermodynamic consistency method, which involves adjusting adsorption/desorption reaction energetics $\left(\Delta \zeta_{\mathrm{i}, \text { ads }}\right)$ to a self-consistent surface mechanism and the properties of gas-phase species. While the first method discussed adjusts surface reaction energies to constrain to gas-phase and adsorption/desorption reaction properties ( $\Delta \zeta_{\mathrm{i}, \text { ads }}$ and $\Delta \zeta_{\mathrm{j} \text {,gas }}$ are constant), this method instead alters adsorption/desorption reaction energetics $\left(\Delta \zeta_{\mathrm{i}, \text { surf }}\right.$ and $\Delta \zeta_{\mathrm{j}, \text { gas }}$ are constant). We recommend this method when a surface reaction mechanism is fully defined from first-principle DFT calculations. As discussed by Blaylock et al., the accuracy associated with surface reaction energies is most likely higher than that of adsorption/desorption energies, because reaction energy calculations benefit from partial cancellation of errors between the bonds that are formed and broken (Blaylock et al., 2009). In this methodology, the absolute energy of surface species and transition states should be defined by specific gas-phase references.

This second adsorption-based method can also be incorporated in CHEMKIN (Kee et al., 1991a), albeit in a less straightforward way than the previous method. First, thermochemical properties of adsorbed intermediates must be regressed into NASA polynomials. Second, preexponential factors and activation energies (Coltrin et al., 1991; Kee et al., 1991a) must be defined such that the transition states thermochemical properties (relative to reactant thermochemical properties) are satisfied through forward rate constants. The temperature exponent $\beta$ (Eq. 3) can be fitted to capture the full extent of the temperature dependence of the rate constant.

As an illustration, Figure 5 compares the two methods for the $\mathrm{CH}_{2}{ }^{*}+\mathrm{H}^{*} \rightarrow \mathrm{CH}_{3} *$ reaction on Ni(111). DFT total energy and vibrational frequency results reported by Blaylock et al. were used to calculate the rate constant using method 2. The same DFT information was also used, along with gas-phase thermochemical data from the NIST database (Burgess, 2009), to extrapolate $\Delta \mathrm{H}^{\ddagger}$ and $\Delta \mathrm{S}^{\ddagger}$ at various temperatures using a proximity factor of 0.5 . In this case, reasonable agreement is observed between the two methods. This is because the $\Delta \mathrm{H}_{\mathrm{rxn}}$ computed from the thermodynamic loop through the gas-phase is somewhat similar to the $\Delta \mathrm{E}_{\mathrm{DFT}}$ obtained from the DFT calculations (difference of $4.5 \mathrm{kcal} / \mathrm{mol}$ at $300 \mathrm{~K}$ ). In a more extreme case, Grabow et al. found that the difference between $\Delta \mathrm{E}_{\mathrm{DFT}}$ and $\Delta \mathrm{H}_{\mathrm{rxn}}$ (calculated from the thermodynamic loop through the gas-phase intermediates) at $548 \mathrm{~K}$ for the $\mathrm{COOH}^{*}+\mathrm{O}^{*} \rightarrow$ $\mathrm{CO}_{2} *+\mathrm{OH}^{*}$ on $\mathrm{Pt}(111)$ is $>40 \mathrm{kcal} / \mathrm{mol}$. This would greatly change the DFT calculated activation barrier if method 1 was used.

The use of either thermodynamic consistency method depends greatly on the source of kinetic model's parameters. The following sections discuss various techniques to calculate and estimate thermodynamic and kinetic model parameters.

\subsection{Elementary rate constant estimation from quantum mechanical calculations}


This section summarizes recent methods for estimating rate constant parameters in Eq. 4. Rate constant parameterization follows an important general theme of scientific modeling in that the methods often balance calculation accuracy with expense. This section focuses on firstprinciple techniques followed by a next section where less accurate, albeit less expensive semiempirical techniques are discussed.

The details of quantum mechanical calculations relevant to catalytic kinetic mechanism development are beyond the scope of this review and are covered sufficiently elsewhere (Sholl and Steckel, 2009). Instead, the focus of this section is on translating the output of modern quantum mechanical calculations into surface reaction rate constants. Within the context of mean-field kinetic models, the essential piece of information from a DFT calculation is the total energy $\left(\mathrm{E}_{\text {total }}\right)$. The DFT total energy is a function of the number and type of atoms, volume of cell, and atom configuration. This total energy corresponds to a relative Helmoltz free energy at $0 \mathrm{~K}$, neglecting ZPE. In the case of molecules adsorbed on a 2-dimensional surface, Reuter and Scheffler have shown through dimensional analysis that the PV contribution to the Gibbs free energy can be neglected and thus, the Hemholtz and Gibbs free energies are approximately equal in this scenario (Reuter and Scheffler, 2002).

Additional information is necessary when ZPE and temperature corrections are included in the calculation of relative free energies of different reactant, product and transition states. Specifically, modes of vibration of the adsorbed species are necessary to compute these corrections. Fortunately, modern DFT codes can compute these frequencies accurately.

$$
\mathrm{ZPE}=\sum_{\mathrm{i}} \frac{1}{2} \mathrm{~h} v_{\mathrm{i}}
$$

The ZPE correction is a function of the sum of the product of $1 / 2$, each vibrational frequency $v_{i}$, and Planck's constant $\mathrm{h}$ (Sholl and Steckel, 2009). Obviously, changes in the high frequencies, through reaction (see below) or isotopic labeling (changing the mass of atoms, specifically hydrogen, will alter the frequencies), can significantly affect the calculated energies. Specifically, dehydrogenation mechanisms are greatly affected by ZPE as the frequencies associated with hydrogen stretching modes are usually the largest. Figure 6 shows the energies (referenced from isolated ethanol in vacuum and hydrogen adsorbed on separate slabs) of dehydrogenation products of ethanol on Pt(111) (Salciccioli et al., 2010). The solid line is the energy directly from the DFT calculation. The dotted line includes ZPE corrections for each level of dehydrogenation. As dehydrogenation proceeds, ZPE corrections become more significant.

Temperature dependent properties of intermediates and transition states

Vibrational frequency calculations are also needed for estimating state properties of species at finite temperatures via statistical mechanics (McQuarrie, 1976). The problem then is to define appropriate degrees of freedom for surface species; to this end, several approaches exist.

The simplest approach is to assume that all degrees of freedom are modes of vibration (Jones et al., 2008b). Another method, which takes into account the lateral movement of adsorbates, uses surface diffusion barriers along the directions parallel to the surface to estimate a spring constant used to calculate the two low frequencies associated with frustrated translation (Gokhale et al., 2004; Salciccioli et al.).

$$
\mathrm{q}_{\mathrm{vib}}=\prod_{\mathrm{i}=1}^{3 \mathrm{~N}}\left(\frac{\mathrm{e}^{-\mathrm{hv}_{\mathrm{i}} / 2 \mathrm{k}_{\mathrm{B}} \mathrm{T}}}{1-\mathrm{e}^{-\mathrm{hv}_{\mathrm{i}} / \mathrm{k}_{\mathrm{B}} \mathrm{T}}}\right)
$$


With this methodology the number of relaxed atoms $(\mathrm{N})$ in the DFT calculation will dictate the number of vibrational frequencies $\left(v_{\mathrm{i}}\right)$ to include in Eq. 21. This method allows for a partition function made up of only vibrational contributions from harmonic oscillators (Blaylock et al., 2009). This is a low temperature/high binding energy simplification and assumes that barriers to diffusion and rotation are greater than the thermal energy.

Recent work by Blaylock et al. has fully taken into account rotation and translational movement of more weakly bound surface species (Blaylock et al., 2009). This particular method assesses the barrier of surface diffusion of an adsorbate, as well as the barrier of Z-axis rotation (normal to the surface), and compares these values to $\mathrm{k}_{\mathrm{B}} \mathrm{T}$. If the thermal energy exceeds these barriers, the vibrational modes that correspond to these frustrated translations and rotations are identified and replaced with the appropriate partition function (Blaylock et al., 2009).

$$
\begin{aligned}
\mathrm{q}_{2 \mathrm{D}-\text { trans }} & =\left(\frac{2 \pi \mathrm{Mk}_{\mathrm{B}} \mathrm{T}}{\mathrm{h}^{2}}\right) \mathrm{N}^{0} \mathrm{~A} \\
\mathrm{q}_{\text {rot }} & =\sqrt{\left(\frac{8 \pi \mathrm{Ik}_{\mathrm{B}} \mathrm{T}}{\sigma_{\mathrm{s}}{ }^{2} \mathrm{~h}^{2}}\right)}
\end{aligned}
$$

This method is useful for species that bind weakly to the surface (e.g., water or methane). Eq. 22 is the partition function for the two translational degrees of freedom parallel to the surface and orthogonal to one another. In this expression, $\mathrm{M}$ is the mass of the adsorbate, $\mathrm{N}^{0}$ is the standard state number of binding sites per adsorbate and $\mathrm{A}$ is the surface area per binding site (Blaylock et al., 2009). Eq. 23 can be used as the rotational partition function for free rotations of weakly bound surface adsorbates. Additional information regarding the symmetry number $\left(\sigma_{\mathrm{s}}\right)$ and the moment of inertia (I) are needed for this contribution, both of which can be calculated from the geometry of the molecule. Given the definition of the partition function for each state, the thermochemical properties of each state can be computed, which can be used to compute Arrhenius parameters.

$$
\Delta \mathrm{E}_{\mathrm{i}}=\Delta \mathrm{H}^{\star}=\Delta \mathrm{E}_{\text {Total }}+\Delta \mathrm{ZPE}+\Delta \mathrm{H}_{0, \mathrm{~T}}^{\ddagger}\left(\mathrm{q}_{\mathrm{vib}}, \mathrm{q}_{\text {rot }}, \mathrm{q}_{2 \mathrm{D}-\text { trans }}\right)
$$

The activation energy at a given temperature is equal to the change in enthalpy from the reactant state to the transition state $\left(\Delta \mathrm{H}^{+}\right)$. It is important to note that in the case of surface species, enthalpy and internal energy are approximated as equal due to neglecting the PV contribution. $\Delta \mathrm{E}_{\mathrm{Total}}$ represents the change in total energy of the DFT output from the reactant state to the transition state. The difference in ZPE correction between states, $\triangle \mathrm{ZPE}$, is added to this value. Finally, a correction for temperature is added, $\Delta \mathrm{H}_{0, \mathrm{~T}}^{\ddagger}$, which represents the change of enthalpy from $0 \mathrm{~K}$ to the desired temperature. This final term can be calculated given the selected partition function (it is a function of $\mathrm{q}_{\mathrm{vib}}, \mathrm{q}_{\text {rot }}$ and $\mathrm{q}_{2 \mathrm{D}-\text { trans }}$ ).

For surface reactions, the pre-exponential factor is related to the activation entropy $\Delta \mathrm{S}^{*}$ or the change in entropy from the reactant to the transition state (Houston, 2001). Again, this quantity can be calculated given the specific partition function being used to describe these states.

For adsorption, collision theory is often employed (Blaylock et al., 2009; Dumesic et al., 1993; Grabow et al., 2008; Kandoi et al., 2006; Mhadeshwar and Vlachos, 2007; Salciccioli et al., 2011), which typically contains the sticking coefficient $\mathrm{s}_{\mathrm{i}}$, which is the probability of a gas-phase species coming into contact with the surface and forming a bond. Thus, the pre-exponential for adsorption is given as:

$$
A_{i, \text { stick }}=s_{i}\left(\frac{k_{B} T}{2 \pi M}\right)^{1 / 2}
$$


As a last note, DFT calculations are performed at a certain coverage (e.g., 1/4 or 1/9 for a $2 \times 2$ or $3 \times 3$ unit cell, respectively). As a result, the thermochemistry and reaction barrier do not account for coverage (many-body) effects. They can be thought of as the low-coverage limit that corresponds to the unimolecular or bimolecular rate constants of gas-phase reactions. This idea will be discussed further in a later section (adsorbate-adsorbate interactions).

\subsection{Elementary rate constant estimation from semi-empirical methods}

While semi-empirical methods are rarely as accurate as quantum mechanical approaches, they offer an inexpensive approach to estimating kinetic parameters. This can be extremely useful when mechanisms involve a large number of intermediates and reaction steps. The ability to accurately predict kinetic parameters from thermochemistry can greatly reduce the cost of model development and allow for fast screening of different catalytic surfaces.

Semi-empirical methods relate thermochemical properties of surface adsorbates to atomic properties or properties of smaller units that make up the adsorbate and draw on properties from the corresponding gas-phase molecule. Additionally, semi-empirical methods are often used to correlate thermochemical parameters with kinetic parameters.

Bond-order conservation (BOC) or unity bond index-quadratic exponential potential (UBIQEP) method

A useful approach, applicable to small molecules, is the bond-order conservation (BOC) or unity bond index-quadratic exponential potential (UBI-QEP) technique (Shustorovich and Sellers, 1998). This technique allows for the estimation of molecular binding energies from atomic binding energies and gas-phase bond dissociation energies. Activation barriers are estimated by minimizing the energy of a two-body adsorbate configuration modeled through a Morse potential (Shustorovich and Sellers, 1998).

BOC was originally used to predict activation energies of various elementary steps in order to provide insights into possible pathways, the RDS, and compare various metal catalysts. BOCbased microkinetic modeling was introduced by Vlachos and co-workers in 1999 and found that, with reasonable parameter adjustment, the method can describe quantitatively a breadth of experimental data (Park et al., 1999). The double mapping from atomic biding energies to molecular binding energies and from molecular binding energies to activation energies, shown in Figure 7, enables the estimation of entire microkinetic model parameter sets from just atomic binding energies, a technique that has proven extremely powerful (Hansgen et al., 2010; Kuz'min and Zeigarnik, 2004; Maestri et al., 2009; Maestri et al., 2008; Mhadeshwar and Vlachos, 2005c; Mhadeshwar and Vlachos, 2007; Mhadeshwar et al., 2003; Raimondeau and Vlachos, 2002). A major advantage of the BOC method, besides being inexpensive, is that it automatically ensures thermodynamic consistency at the enthalpic level since the adsorbates form the thermochemical basis from which heat of reactions and activation energies are estimated.

This method has been found to be accurate within $2-4 \mathrm{kcal} / \mathrm{mol}$ when compared to experimentally determined binding and activation energies (Raimondeau and Vlachos, 2002). In addition, the method was found to describe the profound effect of coverage on activation energies and reconcile disparate values reported from different labs (Park et al., 1999). While the limited accuracy of the predicted activation energies was once a deterrent to using BOC, a recently developed variant of this method has successfully addressed this issue, improving the 
accuracy of the predictions at varying coverages (Maestri and Reuter, 2011). Currently, the major drawback of this semi-empirical technique is the questionable accuracy when predicting reaction parameters for larger (specifically multidentate) species, such as those encountered in oxygenates, biomass, Fischer-Tropsch chemistry, etc. In addition, unless one compares BOC predictions to experimental data, one cannot be certain of how reliable predictions are.

Group additivity method for estimation of surface thermochemistry

In order to predict properties of larger adsorbates, group additivity offers an attractive alternative. Originally developed by Benson for predicting thermochemical properties of gasphase molecules (Benson, 1976; Benson and Buss, 1958; Benson et al., 1969), this method approximates the thermophysical properties of a molecule as the sum of properties of its subparts. More recently, this method has been extended to the prediction of properties of hydrocarbons on various transition metal catalysts (Kua et al., 2000; Kua and Goddard, 1998) and oxygenated hydrocarbons on platinum (Salciccioli et al., 2010). These methods are particularly useful for screening out stable adsorbates to hierarchically study with more accurate methods (see glycerol example in Section 5). Unfortunately, the application of these methods to surface intermediates is limited to the group contributions that have been derived, although one can develop new groups at reasonable cost. Additionally, this scheme is limited to thermochemistry and does not predict kinetic parameters for surface reactions. A method for achieving this is described below.

The major benefits of this scheme are the simplicity and negligible computational cost. For example, calculating the $\Delta \mathrm{H}_{\mathrm{f}, 298}$ of the n-butyl radical $\left(\mathrm{CH}_{3} \mathrm{CH}_{2} \mathrm{CH}_{2} \mathrm{CH}_{2}\right)$ adsorbed on Ir amounts to the following summation: $\left[\mathrm{C}-(\mathrm{Ir})(\mathrm{C})(\mathrm{H})_{2}\right]+2\left[\mathrm{C}-(\mathrm{C})_{2}(\mathrm{H})_{2}\right]+\left[\mathrm{C}-(\mathrm{C})(\mathrm{H})_{3}\right]=(-3.73)+2(-4.93)$ $+(-10.20)=-23.79 \mathrm{kcal} / \mathrm{mol}$ (group values from (Kua et al., 2000)). This simplicity is also accompanied by reasonable accuracy. Figure 8 shows a comparison of DFT calculated $\mathrm{H}_{\mathrm{f}, 298}$ to $\mathrm{H}_{\mathrm{f}, 298}$ calculated from group additivity for $\mathrm{C}_{2} \mathrm{H}_{\mathrm{x}} \mathrm{O}_{2}$ and $\mathrm{C}_{3} \mathrm{H}_{\mathrm{x}} \mathrm{O}_{3}$ adsorbed oxygenates (dehydrogenated intermediates of ethylene glycol and glycerol) on Pt (Salciccioli et al., 2010). For many uses, this method offers a great balance between accuracy and expense.

\section{Estimation of entropic parameters}

Entropic contributions to rate constants (pre-exponential factors and sticking coefficients) can be obtained by expensive calculations either through vibrational analysis and TST for activated processes, or molecular dynamics (MD) if the activation barriers are low (Raimondeau and Vlachos, 2002). Further, obtaining the partition function of adsorbed species from first principles can be difficult, and thus, approximate methods can be useful. It has been shown that temperature dependent properties of surface species can be estimated reasonably from a simple analysis of the change in the degrees of freedom upon adsorption (Mhadeshwar and Vlachos, 2007; Mhadeshwar et al., 2003). Along those same lines, a simple estimation method would entail approximating the entropy associated with an adsorbate as that of the corresponding gasphase molecule minus the entropic contribution from translational degrees of freedom (Grabow et al., 2008; Kandoi et al., 2006; Salciccioli et al., 2011; Santiago et al., 2000):

$$
\mathrm{S}_{\mathrm{i}, \text { surf }}(\mathrm{T})=\mathrm{F}_{\text {loc }}\left(\mathrm{S}_{\mathrm{i}, \text { gas }}(\mathrm{T})-\mathrm{S}_{\mathrm{i}, \text { trans }}(\mathrm{T})\right)
$$

Eq. 26 further includes a factor $\left(\mathrm{F}_{\text {loc }}\right)$, which is the fraction of local entropy of the species retained upon adsorption (Santiago et al., 2000). The value of $F_{\text {loc }}$ has been used as an adjustable parameter in recent microkinetic models (Grabow et al., 2008; Kandoi et al., 2006), and is 
usually close to unity. In case of $F_{l o c}=1, S_{i, \text { trans }}$ is equal to the magnitude of the entropy of adsorption $\left(\Delta \mathrm{S}_{\mathrm{i}, \text { ads }}\right)$ in Eq. 18.

Pre-exponentials can be approximated based on the type of reaction. Dumesic provides order of magnitude estimates of pre-exponential factors based on the class of surface reaction or activation of adsorption (Dumesic et al., 1993). For example, estimates from TST for LangmuirHinshelwood (LH) type reactions are given in Table 6. In this case, information about surface mobility can be used to better approximate pre-exponential factors. Diffusion barriers of surface intermediates can be estimated from the binding energy (Nilekar et al., 2006). In essence, knowledge about the binding energy of adsorbates allows for more accurate estimates of preexponential factors.

Linear scaling relations and transferability of thermochemistry among metals

Once the thermochemical properties of reaction intermediates in a microkinetic model are defined on a single surface, a recent development by Nørskov and co-workers can be used to transfer these energetics to other surfaces based solely on atomic binding energies (AbildPedersen et al., 2007; Fernandez et al., 2008; Jones et al., 2008a). This method correlates molecular heat of chemisorption linearly to the atomic binding energy of the heteroatom binding to the metal, according to (Abild-Pedersen et al., 2007)

$$
\mathrm{Q}^{\mathrm{AH}_{\mathrm{x}}}=\gamma \mathrm{Q}^{\mathrm{A}}+\xi
$$

Here, $\mathrm{Q}^{\mathrm{AHx}}$ is the molecular heat of chemisorption and $\mathrm{Q}^{\mathrm{A}}$ is the (heteroatom) atomic heat of chemisorption. For example, the binding energy of methyl $\left(\mathrm{Q}^{\mathrm{CH} 3}\right)$ can be linearly correlated across metals as a function of the carbon binding energy $\left(Q^{C}\right)$. The slope of this correlation $(\gamma)$ is a function of the valency of the binding atom

$$
\gamma(\mathrm{x})=\frac{\mathrm{x}_{\max }-\mathrm{x}}{\mathrm{x}_{\max }}
$$

where $\mathrm{x}$ is the number of ligands (excluding the metal) of the binding atom. $\mathrm{x}_{\max }$ corresponds to the maximum number of ligands the central atom can take (i.e., $\mathrm{C}=4, \mathrm{~N}=3, \mathrm{O}=2$ ). For example, the slope of the $\mathrm{CH}_{3}$ relationship will be (4-3)/4 $=0.25$. The intercept, $\xi$, can be set from knowing the adsorbate binding energy on one metal.

This method can be extended to multidentate intermediates (Jones et al., 2008a; Salciccioli et al., 2010), an important step toward developing microkinetic models of larger molecules, by taking into account second-order effects, specifically the weak oxygen-metal interactions and strain effects caused by scaling to different sized lattices. With these corrections, it was shown that this linear scaling method can predict the adsorption of $\mathrm{C}_{2} \mathrm{H}_{\mathrm{x}} \mathrm{O}_{2}$ intermediates on $\mathrm{Ni}(111)$ and Ni-Pt-Pt(111) bimetallic surface from only the adsorption energies on $\mathrm{Pt}(111)$ (Salciccioli et al., 2010). Parity plots showing the comparison of these scaling predictions to DFT calculated energies of adsorption are shown in Figure 9. An obvious limitation of this method is its inability to predict transition state properties directly from the equations above. However, with the assumption that the transition state structure is constant from surface to surface, the energy of the transition state should be a linear function of atomic binding energies for the transition state atoms which interact to the surface (Falsig et al., 2008).

\section{DFT-based linear free energy or Brønsted-Evans-Polanyi (BEP) relations}

Brønsted-Evans-Polanyi (BEP) relationships or linear free energy relationships (when associated with Gibbs free energy) enable the prediction of kinetic properties from thermochemical properties of adsorbates (Brønsted, 1928; Evans and Polanyi, 1936) (middle box 
of last row in Figure 1). The need for this approach becomes imperative for large reacting systems. Its validity has been known for many decades for gas-phase chemistry but has only recently proved for surface reactions, e.g., (Alcalá et al., 2003; Bligaard et al., 2004; Nørskov et al., 2002).

The fundamental aspects of these relationships are discussed in depth in recent works (van Santen et al., 2010)

$$
\delta \Delta \mathrm{E}_{\mathrm{i}}=\alpha \delta \Delta \mathrm{E}_{\mathrm{i}, \mathrm{rxn}}
$$

Eq. 29 shows a general relationship where $\delta \Delta \mathrm{E}_{\mathrm{i}}$ is the change in activation energy, $\delta \Delta \mathrm{E}_{\mathrm{i}, \mathrm{rxn}}$ is the change in energy of reaction and $\alpha$ is a constant related to the specific classification of a reaction. Essentially, this model offers a way to predict the activation energy of a reaction using the energy of reaction, from the correlation developed from similar reactions that belong to the same homologous series (these relationships have been used for free energies as well as enthalpies). The idea is to perform a small number (ideally only 2; practically 3-4) TS searches for a homologous series, i.e., reactions that have a similar TS, such as hydrogen abstractions from $\mathrm{C}$, $\mathrm{C}-\mathrm{C}$ bond scissions, etc. Using such linear free energy relations, one can determine the parameters of the rest of the reactions in each homologous series from the thermochemistry. Such relationships have been developed in recent literature for a variety of chemistries on many metals including but not limited to diatomic gas dissociation (Cheng et al., 2008; Falsig et al., 2008; Gajdos et al., 2006; Michaelides et al., 2003; Nørskov et al., 2002) and dehydrogenation chemistry (Chen and Vlachos, 2010; Cheng et al., 2008; Garcia-Mota et al., 2010; Michaelides et al., 2003; Wang et al., 2006).

Figure 10 shows an example of this type of BEP relationship taken from (Michaelides et al., 2003), where the activation barriers $\left(\Delta \mathrm{E}_{\mathrm{i}}\right)$ are plotted as a function of the heat of reaction $\Delta \mathrm{H}_{\mathrm{i}, \text { rxn }}$ for $\mathrm{CH}_{\mathrm{x}} \rightarrow \mathrm{CH}_{\mathrm{x}-1}+\mathrm{H}$ reactions over several transition metal surfaces. In this example, the constant $\alpha$ appearing in Eq. 29, is equal to 0.72 .

While the BEP relationship defined by Eq. 29 can be used to describe reaction energetics, a slightly different linear free energy relationship has emerged, which is frequently used. Proposed by Alcalá et al., these types of correlations linearly relate the transition state energy to reactant or product energy, referenced from the corresponding gas-phase energy of the reactant (Alcalá et al., 2003).

The accuracy in estimating the transition state energy has later been shown to improve based on reaction type classification (Loffreda et al., 2009). The schematic in Figure 11 shows the definition of initial state $\left(E_{I S}\right)$, transition state $\left(E_{T S}\right)$ and final state $\left(E_{F S}\right)$ energies for an elementary reaction. An example of a linear relationship between the final state energies and the transition state energies (for methanol dehydrogenation reactions written in the exothermic direction (Greeley and Mavrikakis, 2004b)) is shown in Figure 12. This type of relationship has primarily been used for dehydrogenation reactions (Li et al., 2009; Maestri and Reuter, 2011) and C-C and C-O bond cleaving reactions (Alcalá et al., 2003; Chen and Vlachos, 2010; Li et al., 2009). These reactions are well suited for this type of BEP, because the transition states are generally either very late or very early. The slope of these regressions is generally below but close to one, which implies that the reverse activation energy of reaction is similar for all reactions (assuming that the $\mathrm{x}$-axis is $\mathrm{E}_{\mathrm{FS}}$ ).

Aside from the ability to inexpensively predict somewhat accurate transition state energies from reactant and product energies, a major advantage of these BEPs is their metaltransferability. When coupled with the aforementioned linear scaling relationships, this creates a powerful tool for probing different metals with the same microkinetic model. This is done via a 
scheme that uses the linear scaling relationships to transfer thermochemical properties of intermediates from the original metal to a new metal. Then these scaled thermochemical properties are used in BEP relationships to calculate activation energies on the new metal. Ferrin et al. applied this method to ethanol decomposition to compare the relative activity of several noble metals (Ferrin et al., 2009). Similarly, direct regressions from DFT data on multiple metals can be used to correlate transition state properties to atomic binding energy descriptors (Ferrin and Mavrikakis, 2009; Jones et al., 2008b; Schumacher et al., 2005).

\section{Adsorbate-adsorbate interactions in mean-field models}

The results of microkinetic models can be greatly affected from adsorbate-adsorbate interactions (Getman and Schneider, 2010; Mhadeshwar et al., 2004). An example for $\mathrm{NH}_{3}$ decomposition is shown in Figure 13a. Lateral interactions are typically calculated via DFT or determined from surface calorimetry (Brown et al., 1998; Yeo et al., 1997) or temperature programmed desorption experiments (Masel, 1996). The inclusion of these interactions into a microkinetic model is not trivial. A certain course-graining needs to take place to convert spatially dependent DFT calculated interaction energies into coverage dependent microkinetic model parameters. Even for smaller, monodentate adsorbates, the configurations that produce minimum energy at specific coverages can be hard to predict. In the case of asymmetric, multidentate adsorbates, the existence and location of spectator molecules can markedly influence the predicted energetics. Mean-field microkinetic models approximate a limit of infinite coordination of the surface lattice. On the other hand, DFT calculations have well defined, finite coordination of surface atoms that depend on the material and surface facet. As a result, information based on DFT energies that change with addition of adsorbates cannot be accurately translated into these meanfield models. For lattice KMC models, this detailed spatial information can be retained.

DFT calculations are performed to monitor the change in energy with the addition of adsorbates in the proximity of the structure of interest (whether it be an intermediate or transition state). In general, coverage dependencies of mean-field kinetic model parameters are determined from regressions of several DFT data points. Previous models have included linear functions of surface coverage (Getman and Schneider, 2010; Kitchin, 2009; Mhadeshwar and Vlachos, 2007; Salciccioli et al., 2011) as well as more complicated exponential functions (Grabow et al., 2008; Kandoi et al., 2006). The expense of performing several DFT calculations for such regressions makes a priori application of DFT to all multicomponent interactions impractical. We revisit this topic below (Section 5) where the hierarchical multiscale modeling framework is presented.

\subsection{Analysis of microkinetic modeling results}

Analysis of a microkinetic model is important when applying it to reactor and catalyst design. A posteriori analysis is centered on reaction intermediates and reaction fluxes. Organizing and interpreting this data enables a fundamental understanding of the surface chemistry and descriptors that control it.

The simplest analysis is performed by observing the concentration profiles of surface species as a function of time, location, or environmental condition in a chemical reactor model. The MASIs on the surface give insights into active reaction pathways and specific elementary reactions that can increase global rates. By increasing the temperature or by modifying the catalyst, one can free the occupied surface sites, and thus, lower the surface coverage.

Analyzing the specific elementary reaction rates is also important in gaining a better understanding of the reaction network. Typically, reaction path analysis (RPA) is used to identify the specific sequence of elementary reactions that convert reactants to products. This is 
specifically valuable for selectivity (Grabow et al., 2008; Kandoi et al., 2006; Maestri et al., 2009; Mhadeshwar and Vlachos, 2005c; Mhadeshwar and Vlachos, 2007; Salciccioli et al., 2011). Eq. 30 shows the general equation for RPA

$$
\chi_{i, j}=\left\{\begin{array}{l}
\frac{v_{i, j} r_{f, j}}{\sum_{k} v_{i, k} r_{f, k}}, v_{i, j}>0 \\
\frac{v_{i, j} r_{b, j}}{\sum_{k} v_{i, k} r_{b, k}}, v_{i, j}<0
\end{array}\right\}
$$

Here $r_{j}$ is the rate of irreversible reaction $j$, $\mathrm{f}$ and $\mathrm{b}$ stand for forward and backward, respectively, and $v_{i, j}$ is the stoichiometric coefficient of species $i$ in reaction $j$ (summation over all reactions $k$ ). $\chi_{i, j}$ is the fraction of production $\left(v_{i, j}>0\right)$ or consumption $\left(v_{i, j}<0\right)$ that reaction $j$ contributes to the flux of species $i$.

Comparing forward and reverse reaction rate pairs is important in identifying elementary reactions that are in $\mathrm{PE}$. $\mathrm{PE}$ identifies fast reactions whose reactants' and products' concentrations are controlled by thermodynamics. A simple way identifying reactions in PE is to compute the $\mathrm{PE}$ ratio of reaction $\mathrm{i}$

$$
\phi_{\mathrm{i}}=\frac{\mathrm{r}_{\mathrm{f}, \mathrm{i}}}{\mathrm{r}_{\mathrm{f}, \mathrm{i}}+\mathrm{r}_{\mathrm{b}, \mathrm{i}}}
$$

Reactions with PE ratios between 0.45 and 0.55 are considered to be in PE for model reduction (Mhadeshwar and Vlachos, 2005a). The PE ratio is also important for identifying irreversible reactions. These are reactions whose rate in one direction is at least two orders of magnitude higher than the reverse direction $(\varphi>0.99$ or $\varphi<0.01)$. When reactions are (partially) equilibrated $(\phi \sim 0.5)$, their net rate is close to zero. In this case, one should also consider the net rates in using Eq. 30 to determine which reactions form and consume each species. It is entirely possible that some slow reactions may affect the fate of species more than fast reactions.

Elementary reaction rates can also be used to identify intermediates in quasi-steady state (QSS). If the rate of change of the species concentration $\left(\mathrm{dC}_{\mathrm{i}} / \mathrm{dt}\right)$ is small (e.g., $1 \%$ is typically used as a threshold) compared to the actual elementary reaction rates in the material balance, the species is considered to be in QSS. This occurs when the elementary reaction rates are much larger than the intermediate's net flux. Neglecting the transient term is useful in model reduction, as it converts differential equations into algebraic equations which can lead to closed form rate expressions that are easily solvable (Mhadeshwar and Vlachos, 2005a; Salciccioli et al., 2011).

Finally, important model parameters can be identified using sensitivity analysis (SA). SA identifies the RDS of reaction mechanisms, i.e., the step(s) that control the overall reaction rate, along with steps that control responses beyond the RDS, such as selectivity, the MASI, a hot spot, etc. Additionally, SA results at various conditions can also be used as input to a principal component analysis (PCA), which is an important tool in model reduction (Mhadeshwar and Vlachos, 2005a). Eq. 32 is the general form of sensitivity analysis used for microkinetic models.

$$
\mathrm{NSC}_{i, j}=\frac{\mathrm{d}\left(\ln \mathrm{R}_{\mathrm{j}}\right)}{\mathrm{d}\left(\ln \mathrm{A}_{\mathrm{i}}\right)}=\frac{\mathrm{A}_{\mathrm{i}} \mathrm{dR}_{\mathrm{j}}}{\mathrm{R}_{\mathrm{j}} \mathrm{dA}_{\mathrm{i}}}
$$

This equation describes a log-normalized sensitivity analysis which allows for head to head comparison of model parameters. In this equation, $R_{j}$ is the measured response, which is an important metric of model performance. For example, conversions of reactants, global reaction 
turnover frequency (TOF), or selectivity to a desired product are common metrics used as a response. $\mathrm{A}_{\mathrm{i}}$ corresponds to the model parameter which is being perturbed to change the response. The specific sign of the normalized sensitivity coefficient $\left(\mathrm{NSC}_{\mathrm{i}, \mathrm{j}}\right)$ also gives information on whether differential increase of the parameter enhances or represses the metric of interest. $A_{i}$ is typically the pre-exponential factor of reaction $i$ or the forward rate constant of reaction $\mathrm{i}$ (these two lead to equivalent results in Eq. 32).

SA indicates which elementary reactions are most important in achieving the desired metric. If the metric is global reaction rates, the RDS is determined. SA can be conducted on any model parameter. For example, the sensitivity of enthalpy of intermediates can be probed to better understand thermodynamically which surface species' stability most greatly affects reaction rates or product selectivity. This can be a valuable analysis for catalyst design by understanding trends of intermediate binding energy on different catalyst surfaces. For instance, one would expect the net reaction rate to exhibit a high sensitivity on the enthalpy of the MASI, since the latter blocks active surface sites. The sign of the corresponding sensitivity coefficient will indicate that a lower enthalpy (a stronger binding energy) results in lower global reaction rates, since the species blocks more active sites.

Application of these analyses is illustrated in a recent publication describing a mechanism for C2 hydrocarbon chemistry on Pt (Salciccioli et al., 2011). The elementary reactions involved in this model are shown in Table 7. PE analysis is shown in Figure 14a. This plot shows that the C-C cleaving of adsorbed ethyl $\left(\mathrm{C}_{2} \mathrm{H}_{5}{ }^{*}\right)$ and adsorbed ethylidene $\left(\mathrm{CHCH}_{3}{ }^{*}\right)$ are the only irreversible active reactions in hydrogenolysis of ethane. The SA of Figure 14b reveals that these $\mathrm{C}-\mathrm{C}$ bond cleaving reactions are rate determining in ethane hydrogenolysis. Figure 15 contains PE analysis and SA for the same mechanism, but in conditions where ethylene hydrogenation is active. The juxtaposition of Figure 14 and Figure 15 illustrates that while one single mechanism can describe multiple chemical processes and conditions, active elementary reactions and sensitive model parameters vary with feedstock composition, process, and operating conditions. More details of this example, including the full RPA, can be found elsewhere (Salciccioli et al., 2011).

\section{Hierarchical multiscale mechanism development}

The inclusion of highest level theory at each scale (Figure 1) is computationally intractable. For this reason, the hierarchical multiscale modeling framework was introduced (Vlachos et al., 2006) the essence of which is a simple, yet powerful procedure. One starts with a sufficiently simple but physically relevant model at each scale and performs a sensitivity analysis (Mhadeshwar and Vlachos, 2005c; Mhadeshwar and Vlachos, 2005b; Mhadeshwar and Vlachos, 2007), i.e., one uses a model at or near the left of the hierarchy of each scale in Figure 1. Upon identification of the important scale and parameter(s), higher level theory can be used to improve the accuracy of the parameter(s) in the relevant scale. The procedure needs to be iterated until the important parameters do not change between iterations. Below we discuss examples of hierarchical multiscale modeling with focus on treating some of the complexity of catalytic reactions (Figure 3) arising from the sheer size of reaction mechanisms of large molecules and the many-body nature of kinetic rate constants.

\subsection{Accounting for adsorbate-adsorbate interactions}

In Section 4.3, we briefly introduced some of the challenges of incorporating the effect of lateral interactions in a microkinetic model. The discrete to continuous mapping from DFT to 
mean-field models and the large number of DFT calculations needed for multicomponent systems limit the a priori estimation of kinetic parameters as a function of coverage. In hierarchical modeling, models without adsorbate-adsorbate interactions (or with interactions included for those species for which parameters are available) are used to probe sensitive parameters and abundant surface intermediates, whereupon the parameters are reassessed via DFT in the presence of the predicted abundant intermediates. Identification of the MASI and important reactions can reduce the computational cost tremendously, since the thermochemistry and reaction barriers of those important parameters can be parameterized by only the coverage of the MASI. This a posteriori analysis can greatly reduce the expense of calculating all possible adsorbate-adsorbate interactions.

Figure 13Figure 13 shows an example of hierarchical model refinement and comparison to experimental data for the ammonia decomposition reaction (Mhadeshwar et al., 2004). In the specific example, the activation energies were estimated using the UBI-QEP method and the refinement was done by incorporating N-N interactions using DFT calculations into the UBIQEP framework. In two iterations, the model was sufficiently refined. The MASI changed considerably between iterations (Figure 13b), indicating the importance of adsorbate-adsorbate interactions.

Recently, attention has been paid to the nature of these interactions (Kitchin, 2009; Mortensen et al., 1998; Stampfl and Scheffler, 1996). Kitchin and co-workers formulated a simple model to predict adsorption energies as a function of surface coverage (İnoğlu and Kitchin, 2010). Additionally, Maestri and Reuters implemented accurate coverage dependencies within the UBI-QEP framework for activation energy prediction (Maestri and Reuter, 2011). Further development of these types of inexpensive predictive models will provide an approximate but a priori method to include the important adsorbate-adsorbate interactions within the microkinetic modeling framework. Upon solution of the microkinetic model, refinement via DFT may follow. 


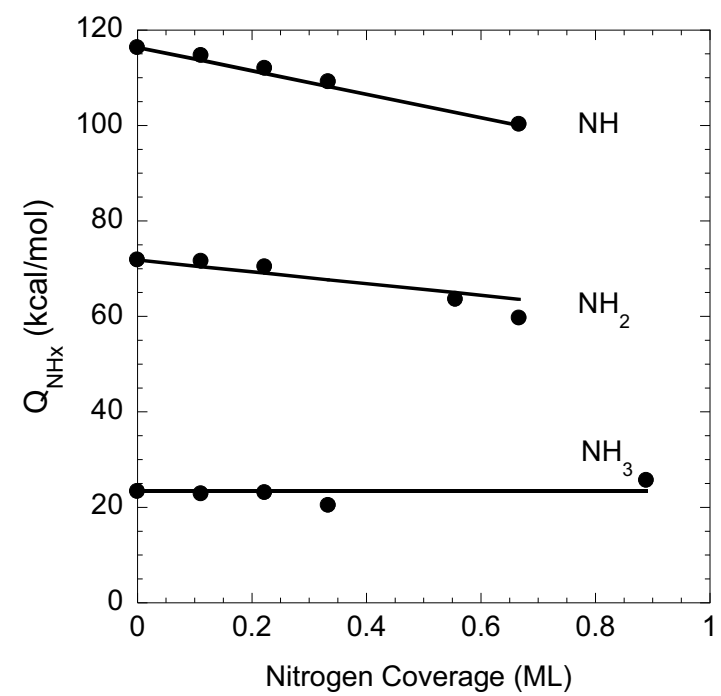

Figure 16 shows the binding energy of various intermediates $\mathrm{NH}_{\mathrm{x}}$ vs. the coverage of $\mathrm{N}$ on a $\mathrm{Ru}$ catalyst in $\mathrm{NH}_{3}$ decomposition. The points are DFT data. The lines are obtained by applying the linear scaling relations (Eq. 27) to account for coverage effects, i.e., by relating the molecular species' binding energies to that of atomic $\mathrm{N}$ where the latter is taken as a linear function of $\mathrm{N}$ coverage from DFT calculations. It is clear that linear scaling relations extend to apply for coverage effects. This approach offers an a priori method to incorporate coverage effects in microkinetic models and has been used in (Hansgen et al., 2010). As suggested above, hierarchical refinement can follow.

\subsection{An example of hierarchical refinement in developing a surface reaction mechanism: Glycerol decomposition on Pt}

A recent example of developing a catalytic kinetic mechanism via hierarchical refinement entails the glycerol decomposition on Pt (Chen et al., Submitted). Figure 17 shows the general methodology followed in developing this mechanism. The overall concept is to use an inexpensive semi-empirical technique to eliminate energetically unreasonable intermediates and reaction paths. This prevents the use of expensive DFT calculations on unimportant mechanistic species and paths. The first hierarchical refinement in this example is on intermediate stability. The second is on reaction barriers.

Essentially, the reaction network for glycerol catalytic decomposition consists of $84 \mathrm{C}_{3} \mathrm{H}_{\mathrm{x}} \mathrm{O}_{3}$ reaction intermediates (glycerol decomposition intermediates). The relative stability of these intermediates was estimated using the group additivity technique for surface oxygenates recently developed (Salciccioli et al., 2010). At this point, 47 stable $\mathrm{C}_{3} \mathrm{H}_{\mathrm{x}} \mathrm{O}_{3}$ surface species were identified using specific energetic criteria (unstable intermediates were omitted). The relative stability of these intermediates was then refined via DFT calculations, to obtain more accurate predictions.

Given the 47 stable (determined via DFT) $\mathrm{C}_{3} \mathrm{H}_{\mathrm{x}} \mathrm{O}_{3}$ intermediates, 101 possible dehydrogenation reactions and 79 possible $\mathrm{C}-\mathrm{C}$ bond scission reactions could occur $(\mathrm{C}-\mathrm{O}$ bond cleaving reactions were left out in this case due to the lack of hydrocarbons in product streams of 
glycerol decomposition experiments) (Davda et al., 2005; Skoplyak et al., 2008a; Skoplyak et al., 2008b). The activation barriers of these reactions were estimated using the BEP relationships. From the results of this semi-empirical analysis, 18 dehydrogenation and $6 \mathrm{C}-\mathrm{C}$ bond cleaving reactions were identified to have reasonably low activation barriers. The energy of the transition states of these reactions was then refined via DFT for a more accurate assessment. From this final refinement, the probable reaction pathways were determined, as shown in Figure 18.

Starting from adsorbed glycerol, a sequence of several dehydrogenation reactions is reasonably likely. Although our DFT results suggest several possible reaction pathways, the most energetically favorable pathway proceeds as follows: $\mathrm{C}_{3} \mathrm{H}_{8} \mathrm{O}_{3} \rightarrow$ $\mathrm{CHOHCHOHCH}_{2} \mathrm{OH} \rightarrow \mathrm{CHOHCHOHCHOH} \rightarrow \mathrm{CHOHCOHCHOH} \rightarrow \mathrm{COHCOHCHOH} \rightarrow$ $\mathrm{COCOHCHOH} \rightarrow \mathrm{CO}+\mathrm{COHCHOH}$. All the reaction barriers in this path are below $0.75 \mathrm{eV}$, consistent with reactions occurring at low temperature (Wang and Liu, 2008). The most likely C$\mathrm{C}$ bond cleaving reaction occurs through the $\mathrm{COCOHCHOH}$ intermediate. In general, - $\mathrm{CO}$ terminated oxygenates exhibit lower barriers for $\mathrm{C}-\mathrm{C}$ bond scission. This has been observed both for glycerol and in a DFT study of ethylene glycol decomposition on Pt (Salciccioli et al., In Press).

Figure 19 shows the estimated computational costs associated with three methods of mechanism development for glycerol decomposition on $\mathrm{Pt}(111)$, and one using only semiempirical methods. The latter carries little to no computational cost; however, the accuracy of kinetic parameters may not give quantitative predictions. The 'DFT' column represents a mechanism which is developed exclusively from DFT calculations. While this type of development results in high accuracy, it is inefficient, as DFT is being used to precisely determine unimportant mechanistic parameters. The middle columns represent hybrid combinations of group additivity and DFT. The two bars shown are the two ends of the spectrum in terms of semi-empirical use and refinement. The first ('screen conformers via GA') is a more conservative approach in which group additivity is only used to screen for the most stable conformers of each adsorbate, and those are used to guide the DFT calculations. While CPU savings are seen with this approach, much higher savings are seen with the method used here ('GA/BEP $\rightarrow$ DFT'), in which only the lowest energy intermediates and pathways are refined via DFT. Not all the model parameters are determined with DFT accuracy but, the most probable reaction parameters are. In a sense, this optimizes resources as seen in Figure 19.

\section{Prediction of novel catalysts via multiscale modeling}

While microkinetic modeling can predict catalyst activity and selectivity and provide mechanistic insights into the RDS and the MASI, its true value is in the design of chemical reactors and/or novel catalysts. The former is congruent with the original objective of multiscale modeling in predicting macroscopic behavior from first principles (Figure 2). Due to the cost of computational fluid dynamics (CFD) simulations and the incompatibility of complex reaction mechanisms with process design software, e.g., Aspen, a posteriori model reduction to closed form rate expressions is a necessity. A simple but powerful methodological approach toward this goal has been presented and examples have been demonstrated for various chemistries, so we do not further review this subject (the interested reader is referred to a tutorial in (Mhadeshwar and Vlachos, 2005a)). Here we focus on an emerging and admittedly more exciting objective, namely use of multiscale modeling for product (catalyst) design. 


\subsection{Catalyst prediction using Sabatier's principle based on a single descriptor}

Sabatier's principle indicates that binding energies of species should be intermediate in magnitude for high activity. Very strong binding energies lead to catalyst poisoning and low reaction rates, whereas very weak binding energies lead to fast desorption, low surface concentrations, and low surface reaction rates. Due to differences in the binding energies of species, it is often the case that one or two species are the most abundant surface intermediates. As a result, the activity of a catalyst is often described in terms of the binding energy of a single species (a single descriptor), as shown in Figure 20. Due to Sabatier's principle, the activity exhibits a maximum at intermediate values of the binding energy, forming a so-called volcano curve.

In the realm of searching for catalysts with improved activity and/or reduced cost, bimetallic catalysts may be used. Typically, a dominant surface species is assumed and an overall LHHW rate expression is derived using a priori assumptions (see Section 3). The reaction rate depends on a single descriptor. By varying the value of the descriptor, a volcano curve can be generated. The values of binding energies of known materials are put on the volcano curve and additional ones can be computed via DFT. The composition of mixed alloys that maximizes activity (max of volcano curve) can be predicted by simple interpolation of the properties of single metals.

The interpolation principle was successfully used to propose a CoMo catalyst in ammonia synthesis from calculations of $\mathrm{N}$ binding on Co and Mo metal slabs. Mo and Co, metals with high and low nitrogen binding energies respectively, were selected to make a catalyst with an intermediate binding energy. After synthesizing this bimetallic catalyst, its activity was found to be comparable to that of the best, but expensive, single metal Ru catalyst (Jacobsen et al., 2001). Another example of this approach includes the prediction of $\mathrm{Fe}-\mathrm{Ni}$ alloys that are superior and less costly to $\mathrm{Ni}$ in the methanation reaction $\left(\mathrm{CO}+3 \mathrm{H}_{2}=\mathrm{CH}_{4}+\mathrm{H}_{2} \mathrm{O}\right)$ (Nørskov et al., 2009).

Overall, current studies have been limited to cases when thermodynamics, specifically the heat of adsorption of a single species, dominates (Greeley and Mavrikakis, 2004a; Strasser et al., 2003) and the concept of linear interpolation applies (Jacobsen et al., 2001). Next, limitations of this approach are discussed and a new framework is proposed that can account for kinetics and transport phenomena in addition to thermodynamics.

\subsection{Overcoming limitations of Sabatier's principle via multiscale modeling}

Prediction of new materials using interpolation principles of the periodic table for mixed alloys and its experimental verification has resulted in considerable excitement. However, there are several limitations of this approach. First, the 'key' surface intermediate has to be guessed. This is not as difficult if the chemistry has been studied for years. For example, in the case of ammonia chemistry, years of research have indicated that $\mathrm{N}$ is a dominant surface species. In the case of ethylene hydrogenation, it is known spectroscopically that ethylidene is an important species. By assuming a dominant species, prediction of materials for which little fundamental research has been conducted is a challenge. In addition, the dominant species may change with operating conditions and/or location in a chemical reactor. Second, with a limited number of exceptions for fairly simple reactions, such as ethylene epoxidation (Linic et al., 2004) and selective acetylene hydrogenation (Nørskov et al., 2009), computational studies have focused on activity. An even more important attribute of a catalyst is its selectivity. Improved selectivity reduces the need for energy intensive separation of products. Maximum selectivity does not follow Sabatier's principle, and as a result, there is no general method for predicting catalyst performance. 
The use of a multiscale model can allow for the prediction of optimal properties in a way that goes beyond a single descriptor and Sabatier's principle, while fundamentally one still uses the interpolation principle of the periodic table. This can be achieved by formulating an optimization problem and explore values of binding energies that maximize or minimize multiple responses (e.g., activity, selectivity, cost, hot spot, etc.). The metal-transferability of the linear scaling relationship, Eq. 27, combined with BEPs provide a mapping from atomic heats of adsorption to activation energies (Figure 7), and thus a means for using microkinetic models for catalyst discovery. This enables one to use atomic or diatomic binding energies as descriptors and probe their optimal values. A full microkinetic model predicts the dominant intermediates and makes no assumptions about PE and RDSs. As a result, it can circumvent problems arising from oversimplification. Finally, the link among scales (i.e., by having a true reactor model) enables one to identify optimal catalyst properties as a function of operating conditions. An example for the $\mathrm{NH}_{3}$ decomposition reaction is shown in

Figure 21. Figure 22 shows examples for $\mathrm{CO}$ oxidation.

\subsection{Design of catalysts with emergent behavior}

There has been recent interest in exploiting 'monolayer' bimetallic catalysts where a second metal is deposited either on the top of another metal (core-shell structure in the case of nanoparticles) or beneath the top layer of the metal. Due to strain and charge transfer effects, the electronic and thus the catalytic properties of these architectures are not linear combinations of those of the parent metals. Rather, they are outside the range of properties (either above or below). Such structures are termed as emergent materials, i.e., their properties cannot be predicted from those of the parent metals. Table 8 illustrates this point through an example for the binding of $\mathrm{N}$ on such architectures.

Recently, a computational platform was developed to predict these novel catalysts for any reaction. First, one develops a microkinetic model for the specific chemistry following the hierarchical methodology discussed above. It is important that semi-empirical methods (e.g., group additivity and BEPs or the BOC method) are used for parameter estimation to allow for transferability of thermochemistry among materials. The microkinetic model is incorporated into a simple reactor model (one-dimensional). Low dimensionality of the reactor model is also important to enable fast calculations. Second, in order to determine optimal atomic binding energies, an optimization problem is formulated, whereby the response of interest (activity, selectivity, etc.) is maximized, subject to thermodynamic constraints (imposed through the microkinetic model as discussed earlier). An example for the $\mathrm{NH}_{3}$ decomposition reaction is shown in Figure 21. Figure 23 and Figure 22 show similar examples for $\mathrm{CO}$ oxidation that will be discussed below.

Having identified optimal binding energies, the next task is to find suitable materials. For this purpose, a library of atomic binding energies is constructed using DFT for various catalyst architectures (surface and sub-surface configurations of various hosts; e.g., $\mathrm{M}_{1}-\mathrm{M}_{2}-\mathrm{M}_{1}$ and $\mathrm{M}_{2}$ $\mathrm{M}_{1}-\mathrm{M}_{1}$ for various combinations of hosts $\mathrm{M}_{1}$ and adlayer $\mathrm{M}_{2}$ metals). This database is subsequently searched to identify a structure (e.g., top, $\mathrm{M}_{2}-\mathrm{M}_{1}-\mathrm{M}_{1}$, or subsurface, $\mathrm{M}_{1}-\mathrm{M}_{2}-\mathrm{M}_{1}$ ) whose properties are close to the optimal ones. An example, using $\operatorname{Pt}(111)$ as the host, is shown in Table 8. This approach does not rely on interpolation of the periodic table and is ideally suited 
to cope with the emergent behavior. As a last step, the materials should be synthesized and tested in the lab and the procedure iterated if need be.

The overall framework is depicted in Figure 24. Application of this framework to the ammonia decomposition reaction led to the discovery of Ni-Pt-Pt (Ni on the top of the Pt host) as an excellent candidate. This was indeed confirmed experimentally and found to have the highest catalytic activity known today for ammonia decomposition to hydrogen for fuel cells (Hansgen et al., 2010).

\section{Uncertainty analysis}

\subsection{Parametric uncertainty}

Kinetic models are subject to various types of uncertainty. The first and most common one is parameter uncertainty. Due to errors in the estimation methods, even when the most accurate DFT methods are employed, kinetic parameters are uncertain. For example, it has been reported that activation energies are estimated with an accuracy of $5 \mathrm{kcal} / \mathrm{mol}$. Pre-exponentials are uncertain within one or even two orders of magnitude. This uncertainty propagates through scales to produce uncertainty of the macroscopic (reactor) scale prediction. Other quantum mechanical method related issues, such as the use of specific functionals for certain species, the lack of spin polarization for certain metals, such as Ni and Co, the lack of good convergence of DFT results, or the use of a small unit cell and a small number of metal layers, can actually cause much larger parameter uncertainty than the error reported above. Due to all these reasons, models usually are not in quantitative agreement with experimental data. That requires model refinement within the uncertainty range. Irrespective of the estimation method, model and experimental uncertainties make parameter refinement a necessity for a quantitative description of experimental data. Systematic refinement methods, using design of experiments, are described in (Aghalayam et al., 2000). In doing so, the refined parameters may not be unique (Prasad et al., 2009) but at least are physically relevant. The number of multiple parameters describing data is much smaller compared to those obtained from fitting with physically unconstrained parameter values.

The development of various mechanisms (e.g., ammonia decomposition on $\mathrm{Ru}$, partial oxidation of methane on Rh, etc.) in our published papers illustrates another point: comparison of microkinetic models to experimental data is essential for model assessment and refinement of key parameters. In fact, while conversion and selectivity data are important macroscopic data to compare to, spectroscopic data are also important in order to interrogate a model. In our experience as a minimum requirement, a model should be able to at least describe the effect of temperature and overall reaction orders well. In addition, catalyst characterization is important to carry out and use its results as input to minimize ambiguity in kinetic parameters arising from surface area effects.

\subsection{Lack of (important) reaction pathways}

Aside from parameter uncertainty, several other possibly more severe issues can hamper the predictive ability of models and overall have to do with lack of correct or complete Physics. The second type of uncertainty is lack of a comprehensive set of elementary steps. For example, in the $\mathrm{CO}$ oxidation chemistry, one typically does not include the reaction $\mathrm{CO}^{*}+\mathrm{O}_{2}{ }^{*} \leftrightarrow \mathrm{CO}_{2}(\mathrm{~g})+$ $\mathrm{O}^{*}+*$, which has been found to be very important on metals such as Au and Ag. Similarly, the old water-gas shift reaction mechanism considers the redox mechanism whereby $\mathrm{H}_{2} \mathrm{O}$ completely 
disocciates to form $\mathrm{O}, \mathrm{H}_{2} \mathrm{O} \leftrightarrow \mathrm{OH}+\mathrm{H} \leftrightarrow \mathrm{O}+2 \mathrm{H}$, followed by the oxidation of $\mathrm{CO}$ by $\mathrm{O}$, $\mathrm{CO}+\mathrm{O} \leftrightarrow \mathrm{CO}_{2}$. However, recent work (Grabow et al., 2008; Mhadeshwar and Vlachos, 2005b) underscores the importance of the carboxyl intermediate, $\mathrm{CO}+\mathrm{OH} \leftrightarrow \mathrm{COOH} \leftrightarrow \mathrm{CO}_{2}+\mathrm{H}$ or $\mathrm{COOH}+\mathrm{O} \leftrightarrow \mathrm{CO}_{2}+\mathrm{OH}$, which is not customarily included in reaction mechanisms. Comparison to experiments may reveal the inadequacy of a model that lacks this chemistry, if the latter is not equilibrated and plays a role in the overall chemical transformation, and guide a modeler into examining novel pathways. Chemical intuition is also important in that regard. An example of including the $\mathrm{COOH}$ intermediate in predicting the strong promoting effect of $\mathrm{H}_{2}$ on $\mathrm{CO}$ in the context of environmental catalysis was recently reported (Hauptmann et al., 2011).

\subsection{Uncertainty due to complexity}

The third and most difficult uncertainty type has to do with the multiscale modeling itself, such as the use of mean-field models instead of the KMC method, the inability of treating stiffness (multiple time scales) in KMC simulations causing the omission of surface diffusion, and the lack of structure-based microkinetic models, such as support and nanoparticle size and shape effects. Currently, it is not generally clear what the impact of incorrect and/or incomplete Physics is. Comparison of models to experiments across scales remains essential in improving our understanding and continuing to build better models. Sections 8 and 9 give some examples of this type of uncertainty.

\subsection{Effect of parametric uncertainty and lateral interactions on catalyst design}

Next, we briefly describe the effect of parametric uncertainty and adsorbate-adsorbate interactions on the identification of optimal catalyst properties, i.e., on the location of the maximum of the volcano curve (in one-dimension) or mountain (in two dimensions). Uncertainty analysis has recently been conducted in a microkinetic model (Ulissi et al., 2010). Various parameters were pertrubed simultaneously using a Monte Carlo search engine. In addition, BOC and BEPs were used to explore the effect of semi-empirical method on predicting the optimal catalyst properies. An example is shown in Figure 25 when lateral interactions are included and excluded. The distributions in each case reflect the effect of an order of magnitude uncertainty in the pre-exponentials. It is clear that while a single value of optimal properties is meaningless, a very tight distribution of optimal properties is predicted. In other words, parametric uncertainty can be ignored in searching for novel materials, as far as a reasonable set of parameters is used. What is interesting, is that lack of lateral interactions shifts the optimal values far way from those predicted when interactions are accounted for. Specifically, if the strong N-N repulsion is neglected, $\mathrm{Ni}$ and $\mathrm{Pt}$ are predicted to be active toward ammonia decomposition. In contrast, when interactions are accounted for, the Ni-Pt-Pt bimetallic is predicted to be active. Experimental results support the importance of lateral interactions that appear, at least for this chemistry, to be critical in the correct identification of suitable catalysts (Hansgen et al., 2010).

\section{Monte Carlo models of chemical kinetics}

\subsection{Historical overview}

The utility of mean-field microkinetic models halts at the necessity to understand spatially localized effects or specific pairwise interactions between adsorbates. While mean-field microkinetic models can be used for catalytic systems with multiple site types, sites are included 
in an average way and are not properly coupled. The physics of multisite or metal-support mediated processes can be lost in mean-field models, as any spatially resolved phenomena require more complex mesoscopic modeling techniques, such as the kinetic Monte Carlo (KMC) method.

The development of spatial KMC methods for simulating discrete events on lattice models has a history that spans several decades. The first spatial KMC algorithm, used to simulate the Ising spin model, is attributed to Bortz et al. (Bortz et al., 1975). Since then, several methodological advances have resulted in a variety of algorithms and frameworks (reviewed in Chatterjee and Vlachos, 2007).

The KMC method became very popular in the 80's and 90's for simulating kinetic phase transitions in model systems (Evans, 1991; Fichthorn et al., 1989; Jensen and Fogedby, 1990; Meakin and Scalapino, 1987; Meng et al., 1994; Zhdanov and Kasemo, 1994; Ziff et al., 1986), investigating the effect of lateral interactions (Myshlyavtsev and Zhdanov, 1989; Silverberg and Ben-Shaul, 1987b; Silverberg and Ben-Shaul, 1987a; Silverberg and Ben-Shaul, 1989; Silverberg et al., 1985; Stiles and Metiu, 1986), and assessing the effectiveness of mean-field models in capturing system behavior close to critical points of the phase diagram (Araya et al., 1989; Dickman, 1986; Dumont et al., 1986; Evans and Miesch, 1991a; Evans and Miesch, 1991b; Jensen et al., 1990; Lutsevich et al., 1991). These works shed light into a variety of interesting phenomena, such as bimodality in temperature programmed desorption (TPD) spectra, noise-induced bistability and stochastic transitions, oscillatory and chaotic behavior in chemical systems, and spatiotemporal pattern formation (reviewed in Albano, 1996; Zhdanov, 2002). However, the majority of these works used simple reaction mechanisms and arbitrary kinetic parameters, and for the most part, they were focused on Physics (non-equilibrium phase transitions) and were disconnected from experimental data. Several nice reviews of the early work on KMC have appeared (Broadbelt and Snurr, 2000; Catlow et al., 1994; Dooling and Broadbelt, 2001; Keil et al., 2000; Lukkien et al., 1998). The KMC method has been applied to metal catalysis as well as zeolites, e.g., (Auerbach, 2000; Coppens et al., 1999; Keil et al., 2000), with the latter focusing more on diffusion and less so on reaction. For this reason, KMC studies in zeolites are not reviewed here.

\subsection{Coupling of KMC method with the bond-order conservation (BOC) method}

In order to introduce a physically relevant methodology for the study of heterogeneous catalysts, the semi-empirical BOC method was introduced to compute activation energies and account for lateral interactions ( $\mathrm{Fu}$ et al., 1999; Lombardo and Bell, 1989; Raimondeau and Vlachos, 2002; Wang et al., 1998). This KMC-BOC method has given its place to the KMCDFT method, described below, due to the increase in computational power and improved accuracy of the DFT calculations.

\subsection{Coupling of KMC method with quantum scale calculations}

In the late 90's and with the advent of first-principles calculations, the KMC method was connected to the quantum-mechanical scale (Figure 26). In the first-principles (or ab initio) $\mathrm{KMC}$, the parameters entering the stochastic rate expressions are obtained through quantummechanical calculations (Figure 27a). Ab initio KMC simulations were originally performed in the context of epitaxial layer growth on metals (Ovesson et al., 1999; Ruggerone et al., 1997) and semiconductors (Kratzer and Scheffler, 2002), and were soon adapted to catalytic processes. 
An overview of the methodology (with one way of interaction between scales) is depicted in Figure 27a. Coupling between scales is discussed below.

Neurock and co-workers were the first to employ such an approach in simulating surface reactions. They introduced a KMC algorithm (Hansen and Neurock, 1999b; Neurock and Hansen, 1998) that takes into account adsorbate binding on different sites (atop, bridge and hollow), and incorporates lateral interactions through either pairwise or the bond-order conservation (BOC) models built from DFT calculations (Hansen and Neurock, 1999a). The algorithm simulates unimolecular and bimolecular reactions, assuming fast diffusion of the adsorbates by equilibrating the lattice after each reaction event. The authors elucidated the effect of interactions on the rates of NO decomposition on $\mathrm{Rh}(100)$ (Hansen and Neurock, 1999a) and showed agreement with experimental data. More recently, detailed simulations of the NO chemistry on Pt nanoparticles were also performed (Mei et al., 2010), showing the interplay between NO oxidation and NO reduction on the different facets (100 and 111) and providing an explanation for the apparent structural insensitivity of $\mathrm{NO}$ oxidation.

Ehylene hydrogenation on Pd(100) was simulated (Hansen and Neurock, 1999b; Hansen and Neurock, 2000a) showing that spatial correlations, stemming from lateral interactions and competitive adsorption, result in apparent reaction orders that are less than unity with respect to hydrogen and negative with respect to ethylene. Mei et al. simulated the selective hydrogenation of acetylene on $\operatorname{Pd}(111)$, predicting the apparent activation energies and reaction orders with respect to hydrogen and acetylene (Mei et al., 2006). This work was more recently extended to ethylene/acetylene mixtures on Pd and Pd-Ag alloys (Mei et al., 2009; Sheth et al., 2005).

Hansen and Neurock simulated the temperature programmed desorption (TPD) spectra of oxygen from $\mathrm{Rh}(100)$ (Hansen and Neurock, 2000b) and found to be in good agreement with experimental data. Simulations revealed that at high coverages, oxygen occupies bridge sites, whereas for lower coverages it binds preferentially to 4-fold hollow sites. Moreover, acetic acid temperature programmed reaction (TPR) spectra calculations (Hansen and Neurock, 2001) showed that the surface was covered by acetate islands surrounded by chemisorbed oxygen atoms that affect the low temperature spectra.

Reuter et al. used $a b$ initio KMC simulation to study the $\mathrm{CO}$ oxidation chemistry on $\mathrm{RuO}_{2}$ (110) (Reuter et al., 2004; Reuter and Scheffler, 2006). The adsorbates can bind to the bridge or cus sites of $\mathrm{RuO}_{2}$. The results obtained from these simulations are in quantitative agreement with experiments and shed light on the relation between the fluctuations on adlayer composition and the catalytic activity. For the same system, Temel et al. compared KMC simulations to mean-field results (Temel et al., 2007). Energetic interactions were neglected and diffusion was accounted for in the KMC simulations, thereby allowing for a fair comparison of the two approaches. The models were in good qualitative agreement; however, the quantitative differences were prominent: the range of partial pressures of $\mathrm{CO}$ where the catalyst is active is much greater in the mean-field model than in the KMC simulations, and the gas composition for which the maximum TOF is achieved is not accurately predicted by this model. Moreover, the reaction rates calculated with the two approaches differ significantly. These discrepancies were attributed to correlations between the positions of vacant sites, introduced solely by adsorptiondesorption and reaction events. Further, Rieger et al. evaluated the effect of surface structure on the TPR spectra for the $\mathrm{CO}$ oxidation on $\mathrm{RuO}_{2}$ in the absence of energetic interactions (Rieger et al., 2008). Their work showed that analysis with mean-field models is inappropriate and can lead to incorrect conclusions on the reactivity of the oxygen atoms that are bound to the two different 
sites (bridge or cus). In another study, Meskine et al. identified the rate-limiting step for CO oxidation on $\mathrm{RuO}_{2}(110)$ (Meskine et al., 2009).

Aiming at bridging the pressure gap, Rogal et al. (Rogal et al., 2008) presented first-principle KMC studies of CO oxidation on $\operatorname{Pd}(100)$. This study indicates the presence of a catalytically active surface oxide structure at ambient pressures and at relevant $\mathrm{CO}: \mathrm{O}_{2}$ ratios. It was concluded that both the reduced $\mathrm{Pd}(100)$ and the oxide phase have to be modeled for a detailed understanding of the $\mathrm{CO}$ oxidation on $\mathrm{Pd}(100)$.

\subsection{Limitations of current KMC simulations}

The studies just reviewed demonstrate that the coupling of KMC with first-principles methods can provide a powerful multiscale modeling framework for quantitative predictions of turnover frequencies, apparent reaction orders and activation energies, as well as TPD and TPR spectra. However, there are still overarching challenges that available KMC codes cannot treat, namely chemical complexity, separation of length and time scales, and heterogeneity of chemical reactivity.

\section{Chemical complexity}

The representation of adsorbate binding and elementary reactions has so far been simplistic. A single site adsorption representation does not account for the multi-dentate nature of several adsorbates (Lukkien, 2009; Meskine et al., 2009; Silverberg and Ben-Shaul, 1987b; Silverberg et al., 1985; Zhdanov and Kasemo, 1998). Furthermore reactions frequently are limited to involve up to two sites. A notable exception is the multisite approach of Hansen and Neurock (Hansen and Neurock, 1999b; Hansen and Neurock, 2000a) that entails reaction detection on the basis of at most two sites, with the possibility of reactants appearing in reflected or intermediate sites. Yet, this approach is inadequate when products appear in sites other than those just mentioned. To overcome this problem, Stamatakis and Vlachos have developed a graph theoretical KMC framework that treats multi-dentate species and arbitrary neighboring and coverage patterns in specifying elementary steps (Stamatakis and Vlachos, Under Review).

The problem then is to define all topologically distinct reactive configurations, since in all current KMC codes elementary reactions are created and imported manually. As the number of adsorbed species and elementary reactions increases, it is impractical to employ ab initio approaches to calculate all rate constants. It might be the case that several of these elementary steps are rarely sampled in a KMC simulation; however, this does not mean that they could safely be omitted since it may be just these rare events that are the most important for the chemistry into consideration.

\section{Separation of scales}

It is typically the case that there is a large disparity between the length- and time-scales in which various elementary events occur. For example, diffusion of mobile species evolves much faster than reactions, rendering $\mathrm{KMC}$ simulation impractical even on the fastest supercomputer, since the algorithm samples almost exclusively unimportant (fast only, e.g., diffusion) events. Similarly, one cannot currently simulate supported catalysts with nanoparticles being far away from each other while accounting for diffusion and possible slow reaction on the support, due to separation of length scales (large distances between nanoparticles). Larger metal nanoparticles are too demanding to simulate with brute-force KMC methods; however, a few sites (e.g., steps) are often actually active but are far from each other (separation of length scales). 
To overcome this multiscale challenge, adaptive mesh coarse-grained Monte Carlo (CGMC) algorithms, having microscopic resolution near edges and corners (where reactivity is higher) and a coarse mesh on flat surfaces, can be employed. This is expected to eliminate error from coarse-graining (or minimize it in the case of chemistry on flat surfaces). An example of adaptivity on a prototype reaction (Figure 28) testifies, for the first time, for the proposed computational method. The proposed adaptivity eliminates the need for domain decomposition, where a deterministic and a KMC model are patched, and alleviates associated problems, such as lack of convergence, mass conservation, and incorrect noise (Schulze et al., 2003), and provides a consistent way to compute ensemble average properties at reduced computational cost.

\section{Heterogeneity of chemical reactivity and many-body effects}

Energetics depends on the local environment of species (adsorbates) in the neighborhood of the microscopic event (see DFT examples in second row of Figure 3). At the global mean-field level, we have successfully incorporated such effects via a hierarchical, albeit lumped, approach (Deshmukh et al., 2004; Mhadeshwar et al., 2004; Mhadeshwar and Vlachos, 2005b; Mhadeshwar and Vlachos, 2005a; Vlachos et al., 2006), whereby the activation energy of important reactions is parameterized as a function of the coverage of the MASI (see Section 4.3).

Adsorbate-adsorbate interactions make the parameterization of the rate constant on a single catalyst site (or a small ensemble of them) a many-body problem whose solution leads to combinatorial explosion in the number of DFT calculations needed. As an example let us consider the parameterization of the Ising Hamiltonian (which assumes only pairwise additive interactions) for $\mathrm{m}$ species on a regular lattice:

$$
\mathrm{H}(\boldsymbol{\eta})=\mathrm{h}_{0}+\sum_{\mathrm{k}=1}^{\mathrm{m}} \sum_{\mathrm{i}=1}^{\mathrm{N}} \mathrm{h}_{\mathrm{k}}^{(1)}\left(\mathbf{x}_{\mathbf{i}}\right) \cdot \eta_{\mathrm{i}, \mathrm{k}}+\sum_{\mathrm{l}=1}^{\mathrm{m}} \sum_{\mathrm{k}=1}^{\mathrm{m}} \sum_{\mathrm{j}=1}^{\mathrm{N}} \sum_{\substack{\mathrm{i}=1 \\ \mathrm{i} \neq \mathrm{j}}}^{\mathrm{N}} \mathrm{h}_{\mathrm{k}, 1}^{(2)}\left(\left|\mathbf{x}_{\mathbf{i}}-\mathbf{x}_{\mathbf{j}}\right|\right) \cdot \eta_{\mathrm{i}, \mathrm{k}} \cdot \eta_{\mathrm{j}, 1}
$$

where $N$ is the number of lattice sites, $\boldsymbol{x}_{\boldsymbol{i}}$ the spatial coordinates of the $\mathrm{i}^{\text {th }}$ site, $\eta_{i, k}$ is the occupancy which is equal to 1 if site $i$ is occupied by species $k$, and $h_{k}^{(1)}, h_{k}^{(2)}$ are constant coefficients. In order to obtain the latter, one has to perform a number of DFT calculations equal to:

$$
\begin{array}{ll}
n \cdot m \cdot\left(\frac{m-1}{2}+1\right)+1 & \text { for } m>1 \\
n+1 & \text { for } m=1
\end{array}
$$

where $\mathrm{n}$ is the neighbor "depth": 1 for nearest neighbors, 2 for next-nearest neighbors etc. The number of such calculations increases rapidly for complex chemistries. For example in ethylene glycol decomposition chemistry, if one was to include up to next-nearest neighbor interactions, the number of species is roughly $m=30$ and the neighbor depth $n=2$. Thus one has to perform around 1000 DFT calculations. Note that this is just for a rather crude approximation of the Hamiltonian, since the interactions are generally non-additive; clusters of more than two adatoms may have a significant contribution to the total energy and need to be computed. It becomes obvious that computation of the energy of the system after every KMC event (to compute barriers) leads to an intractable problem.

Short-range interactions reduce significantly the number of configurations that need to be computed via DFT. Still, the computational cost remains prohibitive and explains why researchers use either a coverage-independent rate constant (the bimolecular analogue of gasphase kinetics) or include coverage effects via BOC or through a mean-field manner. Needless to say, for large molecules, such as polyols and sugars, there exist also a large number of conformers that are close in energy and should thus be all modeled. 
These challenges necessitate the development of novel approaches that will enable the application of ab initio KMC methodologies in complex chemistries while accurately accounting for the distribution and the conformations of adsorbates. Self-learning KMC techniques (Kara et al., 2009; Trushin et al., 2005), importance sampling (Baggerly et al., 1999), as well as parallelization (Martínez et al., 2011) could be proven invaluable in overcoming the aforementioned obstacles and making $a b$ initio $\mathrm{KMC}$ calculations with bi-directional coupling feasible on a large scale.

Even though the number of possible configurations may in principle be vast, only a handful of configurations are usually important under any set of conditions. This is a result of the dissipative nature of diffusion-reaction problems on surfaces and the competitive nature of adsorbates for catalyst sites, i.e., the difference in binding energies usually results in only one or two species being dominant under any set of conditions. In other words, the system samples only a small hyperspace of the entire space of configurations, reminiscent of low-dimensional manifolds. As a result, the probability distribution function (pdf) of configurations is rather low dimensional. These configurations are unknown a priori. One can develop a computational method, termed novelty sampling (Ludwig and Vlachos, 2007; Ludwig and Vlachos, 2008) shown in Figure 27b, for the parameterization of stochastic simulation via a handful of DFT calculations. Specifically, one can run a KMC simulation to compute pdfs. Subsequently, novel (not in the database) configurations can be passed to the DFT solver to improve their parameters and the KMC simulation must be repeated to identify new candidate configurations. In summary, in the novelty sampling method, a KMC simulation generates an ensemble of configurations, steady state DFT computes the parameters for these configurations, and the procedure is iterated until convergence (e.g., when no new configurations are generated). This approach was recently demonstrated in coupling DFT and molecular dynamics (MD) (Ludwig and Vlachos, 2007; Ludwig and Vlachos, 2008).

Aside from lateral interactions, the reactivity of metal nanoparticles and clusters depends also on the coordination number, on the curvature angle on which the adsorbate binds, as well as on hetero-epitaxial strain and charge transfer, the latter effects being crucial in core-shell bimetallic nanoparticles (Mpourmpakis et al., 2010b). In other words, the rate constant of each catalyst site is often a function of two descriptors, as discussed in the next Section 9. To our knowledge, such effects have never been considered in KMC simulation.

\section{Structure-based microkinetic modeling}

Despite the development of first-principle-based microkinetic models, these models are 'structureless'. They typically use input from a single crystallographic plane, e.g., the (111). As a result, they are strictly applicable to 'ideal' single crystals. In reality, even the (111) plane contains point defects and steps and thus, these models are only an approximation of a true single crystal. They should not be compared to supported catalyst-data, except possibly for structure insensitive reactions.

Supported nanocatalysts bigger than a couple of nanometers consist of distinct crystallographic planes, edges and corners, and the reaction rate is a statistical mechanical average (Figure 1and Figure 2) of the microscopic events happening on all these sites (Figure 3). Until recently, all microkinetic models with single crystal DFT input have been compared to supported catalyst data and their parameters tuned, with reasonable success, to describe data. Obviously, this approach is limited in scope and predictive ability. It is also unclear whether the 
success of mean-field microkinetic models is due to structure insensitivity of a reaction or tuning (even if moderate) of the model parameters to describe data.

The structure sensitivity of a chemical reaction is a fundamental topic in heterogeneous catalysis that has received considerable attention over the years. Yet, little modeling of the structure sensitivity has been carried out and typically with simple reactions and non-first principle-based parameters. In this section, we review examples of mean-field and kinetic Monte Carlo (KMC) studies focusing on real mechanisms and parameters applied to understanding structure sensitivity.

\subsection{Structure sensitivity via mean-field models}

As an example of a semi-empirical based microkinetic model (BEPs developed from DFT; Figure 1) addressing structure sensitivity, the $\mathrm{CO}$ oxidation reaction is considered (Wang et al., 2011, submitted). The mechanism entails the following reversible elementary steps, with the last reaction occurring only on steps (Falsig et al., 2008):

$$
\begin{aligned}
& \mathrm{O}_{2}(\mathrm{~g})+* \leftrightarrow \mathrm{O}_{2}{ }^{*} \\
& \mathrm{CO}(\mathrm{g})+* \leftrightarrow \leftrightarrow \mathrm{CO}^{*} \\
& \mathrm{O}_{2}{ }^{*}+* \leftrightarrow 2 \mathrm{O}^{*} \\
& \mathrm{CO}^{*}+\mathrm{O}^{*} \leftrightarrow \mathrm{CO}_{2}(\mathrm{~g})+2^{*} \\
& \mathrm{CO}^{*}+\mathrm{O}_{2}{ }^{*} \leftrightarrow \mathrm{CO}_{2}(\mathrm{~g})+\mathrm{O}^{*}+* \text { (steps only) }
\end{aligned}
$$

The model considers nanoparticles of octahedral shape, which consist of a single facet type (111), as well as edges and corners. Counting of the sites of each type as a function of nanoparticle size is straightforward. The rate constants on the (111) facets are approximated with those of an infinite (111) plane and the edges and corners with those of (211) steps.

Figure 29 shows the effect of particle size on the reaction rate for $\mathrm{Pt}$ and $\mathrm{Au}$ catalysts at three sets of operating conditions. The observed behavior is complex. In the case of $\mathrm{Au}$, as the particle size increases, the rate per unit site (turnover frequency (TOF)) decreases, indicating that steps are more active than terraces under all conditions. On $\mathrm{Au}$, the $\mathrm{CO}_{2}$ formation channel through $\mathrm{CO}^{*}+\mathrm{O}_{2} *$ (reaction $\mathrm{R} 5$ ) is facile even when $\mathrm{CO}$ covers most of the surface sites.

In contrast, the structure sensitivity of Pt catalyst depends on the operating conditions. Under oxygen-rich conditions and relatively low temperatures, larger Pt particles are more active than smaller ones, indicating that terrace sites are effectively more active than step sites. Under fuelrich conditions and low temperatures, Pt appears as structure insensitive (slight variation of the TOF with size). In contrast, under fuel-rich conditions and high temperatures, larger particles are less active than smaller ones, a feature attributed to steps being more active than terraces. At higher temperatures, a larger fraction of the Pt sites is vacant, and as a result, the reactivity corresponds to the low coverage limit. These simple calculations underscore that the structure sensitivity may depend strongly on operating conditions for some catalysts. This finding may rationalize the apparent discrepancy in the literature of whether the $\mathrm{CO}$ oxidation is structure sensitive or not on some metals. In contrast, for other metals, such as Au, the steps are so much more active than the terraces, that the structure sensitivity is independent of operating conditions. The dependence of structure sensitivity on operating conditions highlights the fact that coverage effects have a profound effect on where the chemistry happens.

Figure 23 shows a volcano map for the $\mathrm{CO}$ oxidation reaction, i.e., the turnover frequency vs. the $\mathrm{CO}$ and $\mathrm{O}$ binding energies, on terraces with and without lateral interactions between adsorbates at $600 \mathrm{~K}$. Figure 22 shows similar plots on stepped surfaces at two temperatures. The 
properties of known materials are also superimposed. It is clear that the optimal properties (indicated with a cross) can vary considerably because of lateral interactions and, for this chemistry, with operating conditions.

As another example, Figure 30 shows modeling results for the effect of particle size and shape on the activity of Ru catalyst toward ammonia decomposition, a reaction that is known to be very structure sensitive (Karim et al., 2009). Modeling results are in very good agreement with experimental data (not shown). The model consists of step (B5) sites and terrace sites. The activity on the former is much higher than that on the latter. Computations and experiments indicate that aside from size, shape can also have an important effect on catalyst activity. The optimum particle size at which the activity reaches a maximum is a strong function of shape, with flat-like nanoparticles of 1-2 monolayers in height exhibiting maximum activity at an unusually large size of $\sim 7.5 \mathrm{~nm}$. Hemispherical nanoparticles exhibit very low reactivity at these large sizes due to their small fraction of B5 sites.

The sensitivity of the volcano map of the $\mathrm{CO}$ oxidation on lateral interactions is consistent with that seen for ammonia decomposition and underscores the need to account for adsorbate interactions in catalytic chemistry (Figure 23). The dependence on operating conditions in the $\mathrm{CO}$ oxidation reaction is not seen in the case of ammonia decomposition. Taken together it appears that if one site is much more active than the rest, the TOF varies monotonically with respect to size. On the other hand, when the activity of various sites is comparable, operating conditions can play a decisive role in the dependence of TOF on size.

As a first step in modeling a distribution of sites of different activity, one can consider the fraction and the reactivity of each site and carry out an area weighted average of the reaction rate using a mean-field microkinetic models. This is obviously an oversimplification of the actual phenomena. The spatial arrangement of sites and the coupling between sites should also be considered. Surface diffusion, which is considered infinitely fast in mean-field models, further couples various sites. This task cannot easily and correctly be handled by a mean-field microkinetic model.

\subsection{Structure sensitivity via KMC simulation}

In an early work, Zhdanov and Kasemo studied the Langmuir-Hinshelwood mechanism of the prototypical reaction $2 \mathrm{~A}+\mathrm{B}_{2} \rightarrow 2 \mathrm{AB}$, in a supported nanoparticle exposing (100) and (111) planes, for which species $\mathrm{A}$ and $\mathrm{B}_{2}$ exhibit different sticking coefficients (Zhdanov and Kasemo, 1998; Zhdanov and Kasemo, 2000). It was thus shown that the $A-B_{2}$ composition window for which the reaction proceeds on the nanoparticle is different from that of an infinite surface. In another study, Zhdanov considered the generic reaction $A+B \rightarrow A B$ in a three-phase system consisting of metal, electrolyte and gas, in the context of electrochemical applications. Species A represents the ion, whereas B is the gas (Zhdanov, 2003). Migration of A to the gas-metal boundary and reaction with $\mathrm{B}$ results in the formation of $\mathrm{AB}$ in the gas phase. According to the Tafel law for this system, the reaction rate should increase exponentially with the electrode potential; however, the $\mathrm{KMC}$ simulations revealed lower reaction rates as a result of diffusion limitations.

Gracia and Wolf developed a KMC method for simulating the generic reaction $A+1 / 2 B_{2} \rightarrow C$ on supported crystallites (nanoparticles), and showed that the crystallite size (for uniformly sized particles) or the distribution thereof has a non-trivial effect on the reaction rate (Gracia and Wolf, 2001). Further, by applying an extension of this method to the $\mathrm{CO}$ oxidation reaction (Gracia and Wolf, 2004) over $\mathrm{Pt} / \mathrm{SiO}_{2}$, they analyzed structure sensitivity as well as thermal effects, and 
demonstrated agreement with experimental data. Qin et al. also studied CO oxidation on supported catalysts, incorporating variable activity of the surface due to an oxidation-reduction phenomenon that results in temporal variations of the $\mathrm{O}_{2}$ sticking coefficient (Qin et al., 1998). It was shown that this phenomenon could explain the self-sustained oscillations observed experimentally for this chemistry. Among first-principle structure-based KMC studies, Mei et al. simulated the NO oxidation and reduction chemistry on a nanoparticle exposing (100) and (111) facets as well as edge and corner sites (Mei et al., 2010). It was shown that NO reduction to $\mathrm{N}_{2}$ occurs primarily on the (100) sites, whereas NO oxidation occurs predominantly on the (111) facets and less intensely on the (100) terraces.

Finally, motivated by the mean-field studies on the structural sensitivity of $\mathrm{CO}$ oxidation presented, we performed kinetic Monte Carlo (KMC) simulations for this chemistry on Au surfaces with different step site densities. These surfaces are summarized in Table 9 and for illustration purposes, the $\mathrm{Au}$ (533) surface and the corresponding KMC lattice are shown in Figure 31. We assume that all surface species $\left(\mathrm{CO}^{*}, \mathrm{O}_{2} *, \mathrm{O}^{*}\right)$ bind to fcc hollow sites, and we distinguish two types of sites, namely steps and terraces. The reaction mechanism of (Wang et al., 2010) was used, along with the parameters and energetics corresponding to Au (also shown in Table 10). For simulating this system, the graph-theoretical KMC framework was developed (Stamatakis and Vlachos, Under Review).

A snapshot from these simulations, pertaining to the $\operatorname{Au}(533)$, is shown in Figure $32 \mathrm{a}$, in which the step sites are denoted by squares and the terrace sites by circles. The surface is primarily covered by molecular oxygen followed $\mathrm{CO}$ and $\mathrm{O}$ in comparable coverages. It is worth noting that $\mathrm{O}_{2}$ dissociation happens predominantly on step sites. As a result, the computed turnover frequencies (TOF) per monolayer scale linearly with respect to the step site density, as shown in Figure 32b, whereas the $\mathrm{Au}(111)$ surface that lacks step sites is practically inactive. Thus, the steps are the active sites for the CO oxidation chemistry on gold, consistent with the mean-field microkinetic model. If the step site density is mapped to an equivalent ratio for octahedral Au nanoparticles, then the TOF can be plotted with respect to an equivalent particle diameter, in line with the mean-field microkinetic model.

\subsection{Thermochemical properties on nanoparticles and clusters}

Periodic (plane wave) slab DFT calculations can be applied on single crystals (infinite surfaces, such as the (111) plane). However, catalyst particles consist of flat surfaces, edges, and corners (multiple length scales). It is generally known that steps (and by extension corners) are more active than flat surfaces, provided that they are not poisoned. In order to simulate the chemistry on nanoparticles, one can perform DFT calculations on various local environments (e.g., on each metal atom having a different number of nearest neighboring metal atoms - termed also the coordination number $(\mathrm{CN})$ ). DFT simulations (inset of Figure 33) indicate that one can relate the binding energy, $\mathrm{Q}_{\mathrm{k}}$, of species $\mathrm{k}$, with the $\mathrm{CN}$, i.e., $\mathrm{Q}_{\mathrm{k}}=\mathrm{Q}_{\mathrm{k}}(\mathrm{CN})$. With thermochemistry at hand, one can estimate activation energies on each site of the nanoparticle (e.g., flat surfaces, edges, corners), accounting for the variation of the $\mathrm{CN}$ with particle size and shape using BEPs or linear free energy relations. Currently, such an applicability of BEPs on nanoparticles has not been proven. Smaller nanoparticles $(<1-2 \mathrm{~nm})$ have no well-defined structure. They consist exclusively of undercoordinated sites. In this case, one has to develop models that explicitly depend on the catalyst structure. One recent example considered the $\mathrm{CO}$ binding on Au clusters supported on $\mathrm{MgO}$. By considering a database of clusters in quantum calculations of $\mathrm{n}=6,12$, and 20 atoms (Mpourmpakis and Vlachos, 2009) and 16, 18, 19, 25, 26, 30, 37, and 45 atoms 
(Mpourmpakis et al., 2010a), i.e., up to $1.2 \mathrm{~nm}$, via massive DFT calculations using the Turbomole 5.9.0 program, it was found the BE scales linearly with the coordination number, CN, for each cluster (as shown in the inset of Figure 33). This indicates that the CN number is, as expected, a main descriptor of thermochemistry, e.g., of the binding energy. However, in comparing the linear relationship on many clusters, it was clear that the BE varies considerably among clusters for the same coordination, e.g., $\mathrm{CN}=6$, by as much as with the $\mathrm{CN}$, as shown in the main Figure 33. This indicates that the development of a physicochemical model requires at least one more descriptor beyond the $\mathrm{CN}$ to describe the effect of local environment on thermochemistry. In exploring various secondary descriptors, it was found that the curvature angle of the plane of $\mathrm{Au}$ on which $\mathrm{CO}$ binds is important. Biquadratic models adequately fitted the DFT data. Application of these nanoparticle models to predict reactivity and compare to experimental data will be valuable.

\section{Catalyst dynamics}

Most first-principle calculations assume a static picture of the catalyst that is typically observed in microscopy studies or determined from the Wulf plot of thermodynamic stability. In reality, catalysts are dynamic and respond to their external environment by changing size and/or shape. Dynamics is a hard, multiscale problem, whereby phase transitions and reconstructions occur over second to minute time scales, well beyond the nanosecond realm of molecular dynamics modeling. Below we describe a first attempt on understanding catalyst dynamics subject to annealing with an example on bimetallic catalysts.

\subsection{An example of combined molecular modeling and experiments for structure determination of bimetallic catalysts}

Bimetallic catalysts have recently become the focus of attention due to their higher activity and selectivity compared to their parent metals in several reactions, such as reforming and hydrogenation. Recent studies have elucidated the mechanisms that lead to these desirable properties and explored potential applications of these novel materials (reviewed in Chen et al., 2008). On the other hand, the mechanisms that contribute to structure determination and mixing between two metals and catalyst stability remain largely elusive. Experimental techniques such as scanning tunneling microscopy (STM) and Auger electron spectroscopy (AES) can provide useful information, but detailed characterization of the first few layers and understanding of the dynamics are currently impossible. This need necessitates the use of molecular simulation to gain relevant insight. An example of such studies is presented next.

Motivated by experimental studies on bimetallic catalysts for ammonia decomposition (Hansgen et al., 2010) and reforming (Skoplyak et al., 2008a), Wang et al. employed a multitude of multiscale tools that span a vast range of time-scales (Figure 34) to probe the structure and the mixing of a Ni monolayer (as well as half and two monolayers) deposited on the Pt(111) surface (and stepped surfaces) (Wang et al., 2010). Auger spectroscopy experiments were also performed and indicated that the Ni/Pt mixing at higher temperatures $(600-900 \mathrm{~K})$ occurs on the order of seconds to minutes. At the finer time-scale (order of several nanoseconds), molecular dynamics (MD) simulations were performed using the embedded atom method potential (Daw and Baskes, 1984; Foiles et al., 1986). At low temperatures, the Ni monolayer remains on the surface, and metastable Ni clusters form on the $\mathrm{Pt}(111)$ surface due to the smaller lattice constant 
of Ni compared to that of Pt. At higher temperatures, these structures are dynamic in nature and may play a crucial role in determining the reactivity of the catalyst, as they result in the formation and reconstruction of active sites for the underlying elementary reactions. It was also shown that the rate of mixing increases rapidly with temperature, resulting in the formation of alloys at high temperatures, as evidenced by the coordination numbers of the two atomic species (Figure 35). More specifically, the Pt coordination around Ni (labeled as Ni-Pt) increases with temperature, while that of $\mathrm{Ni}$ around $\mathrm{Ni}$ (labeled as $\mathrm{Ni}-\mathrm{Ni}$ ) decreases, as a result of the $\mathrm{Ni}$ atoms, originally on the surface layer, diffusing into the second layer first and at longer times and/or higher temperatures deeper into Pt. As a result, mixing entails multiple time scales. Simulations indicate that higher temperatures, smaller Ni coverages and the presence of Pt steps (in this case there is inlayer mixing) facilitate mixing between $\mathrm{Ni}$ and Pt. It is expected then that actual single crystals that exhibit defects and nanoparticles with undercoordinated sites will facilitate mixing.

Accelerated MD gave insights on the behavior of the system at longer time-scales (on the order of milliseconds), whereas the infinite time behavior was investigated through simulated annealing. Both simulation methodologies showed that the system reaches a well-mixed state, thereby forming a Ni/Pt solid solution. Thus, the surface configuration seen at low temperatures is metastable (kinetically trapped).

Nudged elastic band (NEB) calculations provided a static picture of the potential energy surface and elucidated possible pathways for $\mathrm{Ni}$ diffusion in the subsurface (Figure 36). Combined with transition state theory, NEB can provide insights on the timescale of mixing. Specifically, the energy barriers of various mechanisms contributing to $\mathrm{Ni}$ diffusion in the $\mathrm{Pt}$ bulk were found to be on the order of $2 \mathrm{eV}$ resulting in a timescale of mixing on the order of seconds, which compares well with experimental data. Further, by means of NEB calculations and analysis of the MD trajectories, using the van Hove correlation function, it was demonstrated that Ni migration occurs through correlated hops. Thermal fluctuations create holes in the surface of Pt into which Ni diffuses.

While these multiple simulation methods, pertaining to different time-scales, have not been coupled, valuable information of the structural characteristics of a catalyst can still be developed. More studies along these lines will be valuable, especially if linked with predicting the effect of structure on catalyst performance.

\section{Free energy calculations and accelerated molecular dynamics for catalysis in condensed phases}

The majority of first-principle studies in catalysis have considered reactions on metals in vacuo. While lessons learned from these studies have been invaluable for solid-gas heterogeneous catalysis, as the previous sections made the case, a number of reactions occur in solution phase where solvent effects are important and cannot be ignored. A notable example is biomass processing. Due to low thermal stability and volatility of biomass derivatives, such as glucose and fructose, selective catalysis must be carried out in an aqueous phase. For these reactions, different computational tools (beyond periodic DFT calculations) are needed, which are traditionally used in other research areas. In this section, we provide an overview of these computational tools.

Understanding of physical or chemical phenomena in condensed phases (including metal surfaces discussed above) requires knowledge of the free energy landscape. The stable basins, the separating saddle points and the connecting reaction paths are essential to understand the direction and mechanism of the process, as well as the relative stability of the various states of 
the system. Knowledge of the mechanism is important, as it directs the thinking about how to modify the reaction under study, how to carry out the reaction with a solvent that is environmentally (more) benign, how to modify the catalyst being used so as to make the process technologically more attractive (i.e., lower cost), or even how to create a new reaction - all very valuable to emerging technologies, whose viability and sustainability very often hinges upon tight process optimization, availability of resources and adherence to environmental imperatives.

Lower bounds to the time scales for the decay of states of metastability (i.e., for reactivity) may also be determined, as, within the framework of Classical Transition State Theory, one can obtain an upper bound to the rate constant for barrier crossing:

where is the activation free energy, is Boltzmann's constant, is Planck's constant, and is temperature. For systems with only a few degrees of freedom, activation free energies are usually (and routinely) obtained within the harmonic approximation, which entails mapping out the ground, adiabatic potential energy surface (PES) of the system - by means of electronic structure calculations in vacuo - and subsequent normal mode analysis at the critical points. The difficulties (and theoretical limitations) of this approach, even for gas-phase reactions, are wellunderstood. The PES of even the simplest chemical reaction has dimensionality larger than 2 and computing it can become a daunting task. For complex systems, like reactions in solution, such approach is inapplicable and theoretically unfounded. Structures optimized at $0 \mathrm{~K}$ do not represent finite temperature conformational distributions; and solvent dynamics effects are suppressed. Optimized structures of the reactant complex that include a number of explicit solvent molecules, say, water, can be specious: For one, because the stationary conformations of the multi-dimensional surface are not guaranteed to be the optimal ones. Also, because at $0 \mathrm{~K}$ water (or any other solvent for that matter) is hardly in the liquid phase. In fact, as one includes more of these explicit waters in the calculation, emergent properties of the corresponding thermodynamic state may become more prominent. In condensed phases, one must sample the phase space and map out a free energy surface (FES). The tool for that is mainly molecular dynamics (MD).

By way of example, in Figure 37, we show the free energy profile for hydride transfer during the multi-step, acid-catalyzed dehydration of D-fructose to 5-hydroxymethyl-furfural (HMF) in water. We have recently shown that the reaction proceeds through a series of steps that involve hydride and proton transfers. In the particular step shown in Figure 37, the free energy profile is analyzed in the internal energy and entropy terms and we see that, in most part, the change in the total internal energy is responsible for the free energy barrier. What is more interesting, however, is that the internal energy barrier is almost entirely due to solvent re-organization. We arrive at this conclusion by writing the internal energy in terms of contributions from the quantum mechanical energy of the reacting system and interactions of type solvent-solvent and solventreacting system. In this study, the free energy calculations were performed using hybrid Quantum Mechanics/Molecular Mechanics MD simulations and biased sampling (Caratzoulas and Vlachos, 2011). The picture that would emerge if we merely looked at the curve , which depicts the change in the quantum mechanical energy of the reacting system alone, would be different - physically incomplete and quantitatively inaccurate. The effects of solvent dynamics would be missing. This example of homogeneous catalysis indicates that these simulations are feasible and lead the way to computationally design, aside from the catalyst, also the medium (solvent). 
Free energy calculations have a long history and, here, we can merely scratch the surface. The objective is the obvious one: to calculate a FES with the right dynamical content to describe a complex system. The problem is twofold. (i) How to reduce the dimensionality of the system by identifying the slow dynamical variables - also referred to as the problem of reaction coordinates. (ii) How to accelerate the dynamics in order to access time scales associated with so-called rare events, i.e., the problem of the statistically significant sampling of the high-energy regions of phase space that separate FES basins of metastability with long lifetimes, on the order of microseconds and longer.

Methods that heavily rely on the choice for reaction coordinates to accelerate the dynamics can be categorized as umbrella or importance sampling methods (Frenkel and Smit, 2002). They apply a biasing potential in the prescribed reaction coordinates with the intent to "lift" the stable basins of the energy landscape, where an MD trajectory may indefinitely get trapped, and thus help the trajectory explore regions of phase space in the vicinity of the barrier; these are the regions where the dynamics decides the fate of the trajectory, namely, whether it will roll downhill towards the products basin or back to where it started. Choosing a functional form for the bias potential is not a trivial matter. Nevertheless, it is open to one's ingenuity, so long as it is computationally efficient and effective in helping the trajectory escape from deep valleys; a commonly used form is that of overlapping parabolic potentials. Notable also is Voter's choice in his hyperdynamics MD method, where a more "global" approach to the biasing potential is taken (Voter, 1997a; Voter, 1997b). Umbrella sampling techniques can give accurate FESs for multi-dimensional reaction coordinates. A more recent development in this front is Parrinello's metadynamics method (Laio and Parrinello, 2002), a very promising approach that deserves special mention and for that we shall return to it shortly. One may even dispense with the biasing potential altogether by simply constraining the system at fixed values of the reaction coordinate and then carrying on with the sampling. This approach goes by the name constrained dynamics and employs thermodynamic integration to obtain the potential of mean force from the ensemble-averaged constraint forces. For multi-dimensional reaction coordinates the method becomes cumbersome. A popular variant of it is called steered MD and is based on Jarzynksi's equality, which allows the calculation of potentials of mean force from non-equilibrium trajectories (Jarzynski, 1997).

The choice of reaction coordinates poses many challenges, theoretical and practical, especially in complex systems where the slow dynamics is in collective modes that involve slow environmental coordinates, such as solvent re-organization during a chemical reaction in solution, the participation of active site residues in enzymatic catalysis, or strong coupling between the reactant complex and low frequency surface phonons of metal catalyst, etc. In the late nineties, Dellago, Bolhuis and Chandler came up with a method that addresses one of the fundamental questions of reaction rate theory in condensed phases: If we know the location of the bottleneck, the dynamics of the rare event is solved by initiating trajectories from that bottleneck. But what if the bottleneck is not known? And, worse, what if it is not even specifiable in terms of a small number of coordinates? How then can rare events be studied? Their method, named Transition Path Sampling (TPS) (Dellago et al., 1998), solves the problem by requiring as only input an order parameter that uniquely identifies the reactant and product metastable states and an initial path that connects them, the latter usually obtained by Nudged Elastic Band calculations. It does not require a priori knowledge of saddle points, nor does it require the definition of a reaction coordinate, and for that matter it dispenses with pre-conceived notions about the slow dynamical variables of the system. One no more speaks of a unique 
transition state, but of an ensemble of transition states, harvested via a Metropolis algorithm. It is a hybrid Monte Carlo-Molecular Dynamics method and its power lies in the fact that is captures the true reactive dynamics. As a bonus, a reaction coordinate with the right dynamical content can be extracted by appropriate analysis of the sampled reactive paths. It is a computationally demanding method.

Parrinello's metadynamics is, in essence, an umbrella sampling technique, but an adaptive one at that. It addresses the hard problem of constructing a good biasing potential. It does so by considering the history of the system - where it appears to be spending most of its time - and by improving the bias on the fly. It permits to sample, in a seamless way, the FES in a set of prescribed reaction coordinates. The idea is to extend the phase space so as to include the reaction coordinates as additional dynamical variables that carry mass and are restrained by harmonic springs to move in the vicinity of the manifolds defined by the reaction coordinates. By also including in the Lagrangian a history-dependent biasing potential (a non-Markovian term in the reaction coordinates), the trajectory is discouraged from visiting regions of configuration space that it has already explored. The non-Markovian term consists of repulsive, Gaussianshaped potentials that are "dropped" on top of the underlying FES. As the trajectory lingers around a certain region, more and more of the Gaussians are dropped in and as they accumulate they counterbalance the underlying free energy valley, thus allowing the trajectory to escape. This way, the method not only accelerates the simulation of rare events but also maps out the FES as the negative of the sum of the repulsive Gaussians. Unlike TPS, metadynamics is not designed to find the "perfect" reaction coordinate. However, by keep expanding the set of prescribed reaction coordinates, it can, in principle, capture all the relevant dynamical bottlenecks. Naturally, this expansion cannot continue indefinitely, as the method progressively becomes impractical. One of its advantages is how versatile it is with respect to the collective variables one can use to define reaction coordinates (e.g., coordination numbers, the number of hydrogen bonds, potential energy, lattice parameters, etc.). As this list of types of collective variables grows, the scope of applications can only broaden. Currently, metadynamics can effectively compute FESs as a function of three to four collective variables and accelerate the escape from deep local minima with up to four to six collective variables. The method is not a "black box" by any means. The computational efficiency is inversely proportional to the width of the Gaussians raised to the power of the dimensionality of the problem (number of reaction coordinates). On the other hand, the resolution of the FES is no better than the width of the Gaussians. Thus, increasing the resolution has an adverse effect on the convergence properties of the metatrajectory. Furthermore, the mass and stiffness parameters associated with the reaction coordinates must be chosen so as to ensure adiabatic decoupling, namely, that the extra dynamical variables are indeed the slow ones. Given some accuracy requirements, estimating the optimal choice of the parameters involved is not a trivial matter.

While the aforementioned techniques have successfully been applied to enzymatic and liquid phase reactions, their extension to heterogeneous gas-solid and liquid-solid catalytic reactions is just emerging (Vlachos and Caratzoulas, 2010). For example, Pignedoli et al. have recently used hybrid classical/DFT metadynamics simulations to study the mechanism for the dehydrogenation reaction of cyclohexaphenylene at a $\mathrm{Cu}(111)$ surface and to explain experimental findings that want the reaction to proceed only from hydrogen atoms of the "mobile" phenyl groups (Pignedoli et al., 2010). Another example of application of the multiscale QM/MM MD method to acid catalyzed dehydration chemistry was recently reported in (Caratzoulas and Vlachos, 
2011) and free energy calculations in the synthesis of catalytic materials have been illustrated in (Caratzoulas et al., 2006).

\section{Summary and outlook}

The previous decade experienced rapid development of multiscale methodology and algorithms. However, the focus was clearly on method development for prototype systems and less so on application to realistic systems. Given the complexity and emergent behavior of catalytic reactions and reactors, the full impact of multiscale modeling will be materialized if novel tools are developed that should, at least initially, be application- rather than mathematicsor computer science-driven.

This review paper described recent developments and a perspective in multiscale modeling with focus on reaction chemistry and mechanism, i.e., at phenomena spanning from the electronic up to and including the mesoscopic scale. Emerging topics, such as complexity, catalyst dynamics, computation-driven catalyst discovery, emergent behavior in materials design, catalyst structure-based microkinetic models, and chemistry in condensed phase and in particular in solvents were discussed.

Hierarchical multiscale modeling was discussed as an approach to coping with the complexity of realistic systems. The recently introduced DFT-based semi-empirical methods (e.g., linear scaling relations, group additivity, Brønsted-Evans-Polanyi (BEP) relations, metal transferability) provide a powerful framework for estimating thermochemistry and reaction barriers with a minimum number of descriptors (atomic heats of adsorption of elements existing in a reaction mechanism). These semi-empirical methods expand the scope and power of the hierarchical multiscale modeling. The graph-theoretical kinetic Monte Carlo (KMC) method provides a general framework to interface with quantum mechanical calculations and account for the complexity of multidentate adsorbates and multiple types of active sites. It seems that a single structural descriptor (e.g., coordination number) is insufficient for parameterizing the thermochemistry at the nanoscale. Rather, a dual structural descriptor appears to be necessary. Further work will be necessary for a comprehensive understanding of phenomena on $\sim 1 \mathrm{~nm}$ nanoparticles.

Several exciting developments have taken place over the past five years. For example, a systematic methodology has been introduced that enables one to predict novel catalytic materials while accounting for catalyst molecular architecture, catalytic kinetics and reactor effects and cope with the possible emergent behavior of electronic and catalytic properties. As another example, multiscale simulations of fairly large molecules, such as fructose, in solution are entirely feasible. This provides an unprecedented opportunity for understanding the chemistry of biomass processing and eventually improving the catalyst and reaction media. Finally, understanding structure sensitivity via structure-based microkinetic models may enable one to tune the nanoparticle size and shape to improve activity and selectivity.

Despite these exciting developments, there are still many challenges that need to be addressed. While schemes that take advantage of semi-empirical methods, (e.g., linear free energy relationships) are useful for catalyst design, a question that arises is if binding energies are sufficient descriptors given the complexity and multiscale nature of catalytic reactions. Additionally, quantification of the error incurred in applying semi-empirical methods to surfaces that they were not developed on and its impact on understanding the reaction pathways and predicting new materials needs to be addressed. Moving into the realm of complex system feeds (e.g., biomass) and liquid phase processing will require more accurate semi-empirical methods 
and multi-descriptor models for fast prediction of optimal catalysts and reactor conditions. Additionally, shape selective catalysts and supports (e.g., zeolites) will become important in selectively cleaving functional groups and linkages of polymerized feeds. The current microkinetic modeling framework must evolve to predict the complex reaction behavior of these materials. Additional topics need to be accounted for in computation-driven catalyst design, such as the effect of the architecture of the catalyst, the catalyst stability, and the effect of the support. It is also important to quantify the effect of error propagation on multiscale modeling at various scales, along the lines of uncertainty analysis presented in this paper.

An increasing number of studies, in which $a b$ initio or first-principles KMC simulations have been used, has clearly shown that such frameworks have predictive power, stemming from the fundamental description of elementary surface processes at the quantum level, coupled with a statistical mechanical description of the dynamics at the molecular level. The multiscale character of this approach enables one to access a wide range of temporal and spatial scales, and allows for the detailed and accurate modeling of intricacies pertaining to surface chemistries, such as spatial heterogeneities of surface and reactivity, or competition effects and complex dynamical behavior. These effects play a significant role in determining the performance of a catalyst, and can now be understood with the aid of first-principles KMC, thereby making possible the computational design and screening of catalysts and the identification of candidates that can be synthesized and tested in the laboratory. Building published accelerated algorithms from prototype systems to treat multiple length and time scales will further increase the impact of KMC methodologies. It is also clear that the nanoscale structure still imposes challenges in modeling: these are large nanoparticles for direct massive DFT calculations but small enough that quantum finite size effects are important. Parameterization of thermochemistry and kinetics at this scale are still needed.

Finally, the rather limited number of computational studies indicates that at elevated temperatures of relevance to catalytic chemistry, the catalyst structure may be very dynamic. As a result, over the time scale of slow reaction events, an ensemble of structures needs to be considered for estimating the kinetics. Multiscale methods can be subsequently integrated with electronic structure calculations for the determination of the chemical properties of these materials. This integration could provide a comprehensive framework for the computational design and screening of candidate catalytic materials, which can subsequently be tested in the laboratory.

\section{Acknowledgements}

This work has been supported from National Science Foundation (NSF) under grants (CMMI- 0835673; dynamics of catalysts, paper section 10 and CBET-940768; design of emergent materials, section 6) and by the Department of Energy (DOE) under grant (DE-FG0205ER25702; kinetic Monte Carlo methods and structure-based microkinetic modeling, sections 8 and 9). The microkinetic modeling (sections 4 and 5) and condensed-phase calculations (section 11) were based upon work financially supported as part of the Catalysis Center for Energy Innovation, an Energy Frontier Research Center funded by the U.S. Department of Energy, Office of Science, Office of Basic Energy Sciences under Award No. DE-SC0001004. 


\section{References}

Abild-Pedersen, F., Greeley, J., Studt, F., Rossmeisl, J., Munter, T. R., Moses, P. G., Skulason, E., Bligaard, T., and Nørskov, J. K., 2007. Scaling properties of adsorption energies for hydrogen-containing molecules on transition-metal surfaces. Phys. Rev. Lett. 99(1).

Aghalayam, P., Park, Y. K., and Vlachos, D. G., 2000. Construction and optimization of detailed surface reaction mechanisms. AIChE Journal 46(10), 2017-2029, This paper was selected in the highlights of Chem. Eng. Progress (paper of the month), p., Oct. issue, 2000.

Albano, E. V., 1996. The Monte Carlo simulation method: A powerful tool for the study of reaction processes. Heterogen Chem Rev 3(4), 389-418.

Alcalá, R., Mavrikakis, M., and Dumesic, J. A., 2003. DFT studies for cleavage of C-C and C-O bonds in surface species derived from ethanol on $\mathrm{Pt}(111)$. Journal of Catalysis 218(1), 178190.

Araya, P., Porod, W., Sant, R., and Wolf, E. E., 1989. Monte-Carlo Simulations of CarbonMonoxide Oxidation on Pt Catalysts. Surface Science 208(1-2), L80-L90.

Auerbach, S. M., 2000. Theory and simulation of jump dynamics, diffusion and phase equilibrium in nanopores. Int Rev Phys Chem 19(2), 155-198.

Baboul, A. G., Curtiss, L. A., Redfern, P. C., and Raghavachari, K., 1999. Gaussian-3 theory using density functional geometries and zero-point energies. Journal of Chemical Physics 110(16), 7650-7657.

Baggerly, K., Cox, D., Kollman, C., and Picard, R., 1999. Adaptive importance sampling on discrete Markov chains. Annals of Applied Probability 9(2), 391-412.

Benson, S. W., and Buss, J. H., 1958. Additivity Rules for the Estimation of Molecular Properties - Thermodynamic Properties. Journal of Chemical Physics 29(3), 546-572.

Benson, S. W., Cruicksh.Fr, Golden, D. M., Haugen, G. R., Oneal, H. E., Rodgers, A. S., Shaw, R., and Walsh, R., 1969. Additivity Rules for Estimation of Thermochemical Properties. Chemical Reviews 69(3), 279-\&.

Benson, S. W., Thermochemical Kinetics. 2 ed. 1976, New York: John Wiley \& Sons, Inc.

Blaylock, D. W., Ogura, T., Green, W. H., and Beran, G. J. O., 2009. Computational Investigation of Thermochemistry and Kinetics of Steam Methane Reforming on Ni(111) under Realistic Conditions. Journal of Physical Chemistry C 113(12), 4898-4908.

Bortz, A. B., Kalos, M. H., and Lebowitz, J. L., 1975. A New Algorithm for Monte-Carlo Simulation of Ising Spin Systems. Journal of Computational Physics 17(1), 10-18.

Broadbelt, L., and Snurr, R., 2000. Applications of molecular modeling in heterogeneous catalysis research. Applied Catalysis A: General 200, 23-46.

Brønsted, J. N., 1928. Acid and basic catalysis. Chemical Reviews 5(3), 231-338.

Brown, W. A., Kose, R., and King, D. A., 1998. Femtomole adsorption calorimetry on singlecrystal surfaces. Chemical Reviews 98(2), 797-831.

Burgess, D. R., "'Thermochemical Data" in NIST Chemistry WebBook", in NIST Standard Reference Database Number 69, P.J. Linstrom and W.G. Mallard, Editors. 2009, National Institute of Standards and Technology: Gaithersburg, MD.

Caratzoulas, S., Vlachos, D. G., and Tsapatsis, M., 2006. On the role of tetramethylammonium cation and effects of solvent dynamics on the stability of the cage-like silicates Si6O156- and Si8O208- in aqueous solution. A molecular dynamics study. J. Am. Chem. Soc. 128(2), 596606. 
Caratzoulas, S., and Vlachos, D. G., 2011. Converting fructose to 5-hydroxymethyl-furfural: A Quantum Mechanics/Molecular Mechanics study of the mechanism and energetics. Carbohydr. Res. 346(5), 664-672.

Catlow, C. R. A., Bell, R. G., and Gale, J. D., 1994. Computer modeling as a technique in materials chemistry. J. Mat. Chem. 4(6), 781-792.

Chatterjee, A., and Vlachos, D. G., 2007. An overview of spatial microscopic and accelerated kinetic Monte Carlo methods. Journal of Computer-Aided Materials Design 14(2), 253-308, invited.

Chen, J. G., Menning, C. A., and Zellner, M. B., 2008. Monolayer bimetallic surfaces: Experimental and theoretical studies of trends in electronic and chemical properties. Surface Science Reports 63(5), 201-254.

Chen, Y., and Vlachos, D. G., 2010. Hydrogenation of Ethylene and Dehydrogenation and Hydrogenolysis of Ethane on $\operatorname{Pt}(111)$ and $\operatorname{Pt}(211)$ : A Density Functional Theory Study. Journal of Physical Chemistry C 114(11), 4973-4982.

Chen, Y., Salciccioli, M., and Vlachos, D. G., Submitted. An efficient reaction pathway search method applied to the conversion of biomass derivatives on platinum.

Cheng, J., Hu, P., Ellis, P., French, S., Kelly, G., and Lok, C. M., 2008. Bronsted-Evans-Polanyi relation of multistep reactions and volcano curve in heterogeneous catalysis. Journal of Physical Chemistry C 112(5), 1308-1311.

Choi, Y., and Liu, P., 2009. Mechanism of Ethanol Synthesis from Syngas on Rh(111). J. Am. Chem. Soc. 131(36), 13054-13061.

Christofides, P. D., 2001. Control of nonlinear distributed process systems: recent developments and challenges: Perspective. AIChE Journal 47(3), 514-518.

Coltrin, M. E., Kee, R. J., and Rupley, F. M., Surface Chemkin (version 4.0): A FORTRAN package for analyzing heterogeneous chemical kinetics at a solid-surface-gas phase interface. 1991, Livermore, CA: Sandia National Laboratories Report No. SAND90-8003B.

Coppens, M.-O., Bell, A. T., and Chakraborty, A. K., 1999. Dynamic Monte-Carlo and meanfield study of the effect of strong adsorption sites on self-diffusion in zeolites. Chemical Engineering Science 54, 3455-3463.

Curtiss, L. A., Redfern, P. C., and Raghavachari, K., 2007. Gaussian-4 theory. Journal of Chemical Physics 126(8).

Davda, R. R., Shabaker, J. W., Huber, G. W., Cortright, R. D., and Dumesic, J. A., 2005. A review of catalytic issues and process conditions for renewable hydrogen and alkanes by aqueous-phase reforming of oxygenated hydrocarbons over supported metal catalysts. Appl. Catal. B-Environ. 56(1-2), 171-186.

Daw, M. S., and Baskes, M. I., 1984. Embedded-Atom Method: Derivation and Application to Impurities, Surfaces, and Other Defects in Metals. Phys. Rev. B 29(12), 6443-6453.

Dellago, C., Bolhuis, P. G., Csajka, F. S., and Chandler, D., 1998. Transition path sampling and the calculation of rate constants. Journal of Chemical Physics 108(5), 1964-1977.

Deshmukh, S. R., Mhadeshwar, A. B., Lebedeva, M. I., and Vlachos, D. G., 2004. From density functional theory to microchemical device homogenization: Model prediction of hydrogen production for portable fuel cells. International Journal on Multiscale Computational Engineering 2(2), 221-238.

Dickman, R., 1986. Kinetic Phase-Transitions in a Surface-Reaction Model - Mean-Field Theory. Phys Rev A 34(5), 4246-4250. 
Dooling, D. J., and Broadbelt, L. J., 2001. Generic Monte Carlo tool for kinetic modeling. Industrial \& Engineering Chemistry Research 40(2), 522-529.

Drews, T. O., Webb, E. G., Ma, D. L., Alameda, J., Braatz, R. D., and Alkire, R. C., 2004. Coupled mesoscale - continuum simulations of copper electrodeposition in a trench. AIChE Journal 50(1), 226-240.

Dumesic, J. A., Rudd, D. F., Aparicio, L. M., Rekoske, J. E., and Treviño, A. A., The Microkinetics of Heterogeneous Catalysis. 1993, Washington DC: American Chemical Society. 315.

Dumont, M., Poriaux, M., and Dagonnier, R., 1986. On Surface-Reaction Kinetics in the Presence of Islands. Surface Science 169(2-3), L307-L310.

Dyke, J. M., Groves, A. P., Lee, E. P. F., and Niavaran, M. H. Z., 1997. Study of the CH3CHOH radical with ultraviolet photoelectron spectroscopy. Journal of Physical Chemistry A 101(4), 373-376.

E, W., Engquist, B., and Huang, Z. Y., 2003. Heterogeneous multiscale method: A general methodology for multiscale modeling. Phys. Rev. B 67(9), 092101.

Ertl, G., 1991. Oscillatory kinetics and spatiotemporal self-organization in reactions at solid surfaces. Science 254(5039), 1750-1755.

Evans, J. W., 1991. Kinetic Phase-Transitions in Catalytic Reaction Models. Langmuir 7(11), 2514-2519.

Evans, J. W., and Miesch, M. S., 1991a. Catalytic Reaction-Kinetics near a $1^{\text {st }}$-Order Poisoning Transition. Surface Science 245(3), 401-410.

Evans, J. W., and Miesch, M. S., 1991b. Characterizing Kinetics near a $1^{\text {st }}$-Order Catalytic Poisoning Transition. Phys. Rev. Lett. 66(6), 833-836.

Evans, M. G., and Polanyi, M., 1936. Further considerations on the thermodynamics of chemical equilibria and reaction rates. Transactions of the Faraday Society 32(2), 1333-1359.

Falsig, H., Hvolbæk, B., Kristensen, I. S., Jiang, T., Bligaard, T., Christensen, C. H., and Nørskov, J. K., 2008. Trends in the catalytic CO oxidation activity of nanoparticles. Angewandte Chemie-International Edition 47(26), 4835-4839.

Fernandez, E. M., Moses, P. G., Toftelund, A., Hansen, H. A., Martinez, J. I., Abild-Pedersen, F., Kleis, J., Hinnemann, B., Rossmeisl, J., Bligaard, T., and Nørskov, J. K., 2008. Scaling relationships for adsorption energies on transition metal oxide, sulfide, and nitride surfaces. Angewandte Chemie-International Edition 47(25), 4683-4686.

Ferrin, P., and Mavrikakis, M., 2009. Structure Sensitivity of Methanol Electrooxidation on Transition Metals. J. Am. Chem. Soc. 131(40), 14381-14389.

Ferrin, P., Simonetti, D., Kandoi, S., Kunkes, E., Dumesic, J. A., Nørskov, J. K., and Mavrikakis, M., 2009. Modeling Ethanol Decomposition on Transition Metals: A Combined Application of Scaling and Bronsted-Evans-Polanyi Relations. J. Am. Chem. Soc. 131(16), 5809-5815.

Fichthorn, K., Gulari, E., and Ziff, R., 1989. Noise-Induced Bistability in a Monte-Carlo Surface-Reaction Model. Phys. Rev. Lett. 63(14), 1527-1530.

Foiles, S. M., Baskes, M. I., and Daw, M. S., 1986. Embedded-Atom-Method Functions for the Fcc Metals Cu, Ag, Au, Ni, Pd, Pt, and Their Alloys. Phys. Rev. B 33(12), 7983-7991.

Frenkel, D., and Smit, B., Understanding Molecular Simulation: From Algorithms to Applications. 2 ed. 2002, San Diego, CA: Academic.

Fu, G., Xia, W. S., Wan, H. L., and Zhang, Q. E., 1999. Monte Carlo simulation of CO desorption from $\mathrm{Fe}(100)$. Chem J Chinese U 20(3), 440-444. 
Gajdos, M., Hafner, J., and Eichler, A., 2006. Ab initio density-functional study of NO on closepacked transition and noble metal surfaces: I. Molecular adsorption. Journal of PhysicsCondensed Matter 18(1), 13-40.

Garcia-Mota, M., Bridier, B., Perez-Ramirez, J., and Lopez, N., 2010. Interplay between carbon monoxide, hydrides, and carbides in selective alkyne hydrogenation on palladium. Journal of Catalysis 273(2), 92-102.

Gear, C. W., Li, J., and Kevrekidis, I. G., 2003. The gap-tooth method in particle simulations. Physics Letters A 316(3-4), 190-195.

Getman, R. B., and Schneider, W. F., 2010. DFT-Based Coverage-Dependent Model of PtCatalyzed NO Oxidation. Chemcatchem 2(11), 1450-1460.

Gokhale, A. A., Kandoi, S., Greeley, J. P., Mavrikakis, M., and Dumesic, J. A., 2004. Molecularlevel descriptions of surface chemistry in kinetic models using density functional theory. Chemical Engineering Science 59(22-23), 4679-4691.

Grabow, L. C., Gokhale, A. A., Evans, S. T., Dumesic, J. A., and Mavrikakis, M., 2008. Mechanism of the water gas shift reaction on Pt: First principles, experiments, and microkinetic modeling. Journal of Physical Chemistry C 112(12), 4608-4617.

Gracia, F., and Wolf, E. E., 2001. Monte Carlo simulations of the effect of crystallite size on the activity of a supported catalyst. Chemical Engineering Journal 82(1-3), 291-301.

Gracia, F. J., and Wolf, E. E., 2004. Non-isothermal dynamic Monte Carlo simulations of CO oxidation on Pt supported catalysts. Chemical Engineering Science 59(22-23), 4723-4729.

Greeley, J., and Mavrikakis, M., 2004a. Alloy catalysts designed from first principles. Nature Materials 3(11), 810-815.

Greeley, J., and Mavrikakis, M., 2004b. Competitive paths for methanol decomposition on Pt(111). J. Am. Chem. Soc. 126(12), 3910-3919.

Hansen, E., and Neurock, M., 1999a. Predicting lateral surface interactions through density functional theory: application to oxygen on Rh(100). Surface Science 441(2-3), 410-424.

Hansen, E., and Neurock, M., 2001. First-principles based kinetic simulations of acetic acid temperature programmed reaction on Pd(111). Journal of Physical Chemistry B 105(38), 9218-9229.

Hansen, E. W., and Neurock, M., 1999b. Modeling surface kinetics with first-principles-based molecular simulation. Chemical Engineering Science 54(15-16), 3411-3421.

Hansen, E. W., and Neurock, M., 2000a. First-principles-based Monte Carlo simulation of ethylene hydrogenation kinetics on Pd. Journal of Catalysis 196(2), 241-252.

Hansen, E. W., and Neurock, M., 2000b. First-principles-based Monte Carlo methodology applied to O/Rh(100). Surface Science 464(2-3), 91-107.

Hansen, N., Krishna, R., van Baten, J. M., Bell, A. T., and Keil, F. J., 2010. Reactor simulation of benzene ethylation and ethane dehydrogenation catalyzed by ZSM-5: A multiscale approach. Chemical Engineering Science 65(8), 2472-2480.

Hansgen, D. A., Vlachos, D. G., and Chen, J. G. G., 2010. Using first principles to predict bimetallic catalysts for the ammonia decomposition reaction. Nature Chemistry 2(6), 484489.

Hansgen, D. A., "Bimetallic catalysts for the ammonia decomposition reaction: A combined theoretical and experimental study", Univ. of Delaware, Newark, Ph.D. Thesis, (2011).

Hauptmann, W., Votsmeier, M., Vogel, H., and Vlachos, D. G., 2011. Modeling the simultaneous oxidation of $\mathrm{CO}$ and $\mathrm{H}_{2}$ on Pt - Promoting effect of $\mathrm{H}_{2}$ on the CO-light-off. Applied Catalysis A-General, Accepted. 
Hickman, D. A., and Schmidt, L. D., 1993. Steps in $\mathrm{CH}_{4}$ oxidation on Pt and Rh surfaces: High temperature reactor simulations. AIChE Journal 39(7), 1164-1177.

Houston, P. L., Chemical Kinetics and Reaction Dynamics. 2001, Mineola, New York: Dover Publications, INC. 330.

İnoğlu, N., and Kitchin, J. R., 2010. Simple model explaining and predicting coverage-dependent atomic adsorption energies on transition metal surfaces. Phys. Rev. B 82(4).

Jacobsen, C. J. H., Dahl, S., Clausen, B. S., Bahn, S., Logadottir, A., and Nørskov, J. K., 2001. Catalyst design by interpolation in the periodic table: Bimetallic ammonia synthesis catalysts. J. Am. Chem. Soc. 123(34), 8404-8405.

Jarzynski, C., 1997. Nonequilibrium equality for free energy differences. Phys. Rev. Lett. 78(14), 2690-2693.

Jensen, I., and Fogedby, H. C., 1990. Kinetic Phase-Transitions in a Surface-Reaction Model with Diffusion - Computer-Simulations and Mean-Field Theory. Phys Rev A 42(4), 19691975.

Jensen, I., Fogedby, H. C., and Dickman, R., 1990. Critical Exponents for an Irreversible Surface-Reaction Model. Phys Rev A 41(6), 3411-3414.

Jones, G., Bligaard, T., Abild-Pedersen, F., and Nørskov, J. K., 2008a. Using scaling relations to understand trends in the catalytic activity of transition metals. Journal of Physics-Condensed Matter 20(6).

Jones, G., Jakobsen, J. G., Shim, S. S., Kleis, J., Andersson, M. P., Rossmeisl, J., AbildPedersen, F., Bligaard, T., Helveg, S., Hinnemann, B., Rostrup-Nielsen, J. R., Chorkendorff, I., Sehested, J., and Nørskov, J. K., 2008b. First principles calculations and experimental insight into methane steam reforming over transition metal catalysts. Journal of Catalysis 259(1), 147-160.

Kandoi, S., Greeley, J., Sanchez-Castillo, M. A., Evans, S. T., Gokhale, A. A., Dumesic, J. A., and Mavrikakis, M., 2006. Prediction of experimental methanol decomposition rates on platinum from first principles. Topics in Catalysis 37(1), 17-28.

Kara, A., Trushin, O., Yildirim, H., and Rahman, T. S., 2009. Off-lattice self-learning kinetic Monte Carlo: application to 2D cluster diffusion on the fcc(111) surface. Journal of PhysicsCondensed Matter 21(8), -

Karim, A. M., Prasad, V., Mpourmpakis, G., Lonergan, W. W., Frenkel, A. I., Chen, J. G. G., and Vlachos, D. G., 2009. Correlating Particle Size and Shape of Supported Ru/gammaA12O3 Catalysts with NH3 Decomposition Activity. J. Am. Chem. Soc. 131(34), 1223012239.

Kee, R. J., Rupley, F. M., and Miller, J. A., Chemkin-II: A FORTRAN chemical kinetics package for the analysis of gas phase chemical kinetics. 1991a, Livermore, CA: Sandia National Laboratories Report, SAND89-8009.

Kee, R. J., Rupley, F. M., and Miller, J. A., The CHEMKIN thermodynamic data base. 1991b: Sandia National Laboratories Report, SAND87-8215B.

Keil, F. J., Krishna, R., and Coppens, M. O., 2000. Modeling of diffusion in zeolites. Rev Chem Eng 16(2), 71-197.

Kevrekidis, I. G., Gear, C. W., and Hummer, G., 2004. Equation-free: The computer-aided analysis of complex multiscale systems. AIChE Journal 50(7), 1346-1355.

Kitchin, J. R., 2009. Correlations in coverage-dependent atomic adsorption energies on $\mathrm{Pd}(111)$. Phys. Rev. B 79(20). 
Kratzer, P., and Scheffler, M., 2002. Reaction-limited island nucleation in molecular beam epitaxy of compound semiconductors. Phys. Rev. Lett. 88(3), 036102.

Kua, J., and Goddard, W. A., 1998. Chemisorption of organics on platinum. 2. Chemisorption of C2Hx and CHx on Pt(111). Journal of Physical Chemistry B 102(47), 9492-9500.

Kua, J., Faglioni, F., and Goddard, W. A., 2000. Thermochemistry for hydrocarbon intermediates chemisorbed on metal surfaces: $\mathrm{CHn}-\mathrm{m}(\mathrm{CH} 3)(\mathrm{m})$ with $\mathrm{n}=1,2,3$ and $\mathrm{m}<=\mathrm{n}$ on Pt, Ir, Os, Pd, Rh, and Ru. J. Am. Chem. Soc. 122(10), 2309-2321.

Kuz'min, I. V., and Zeigarnik, A. V., 2004. Microkinetic Modeling of ethane hydrogenolysis on metals. Kinetics and Catalysis 45(4), 561-568.

Laio, A., and Parrinello, M., 2002. Escaping free-energy minima. Proceedings of the National Academy of Sciences of the United States of America 99(20), 12562-12566.

Li, M., Guo, W. Y., Jiang, R. B., Zhao, L. M., and Shan, H. H., 2009. Decomposition of Ethanol on Pd(111): A Density Functional Theory Study. Langmuir 26(3), 1879-1888.

Linic, S., Jankowiak, J., and Barteau, M. A., 2004. Selectivity driven design of bimetallic ethylene epoxidation catalysts from first principles. Journal of Catalysis 224(2), 489-493.

Loffreda, D., Delbecq, F., Vigne, F., and Sautet, P., 2009. Fast Prediction of Selectivity in Heterogeneous Catalysis from Extended Bronsted-Evans-Polanyi Relations: A Theoretical Insight. Angewandte Chemie-International Edition 48(47), 8978-8980.

Lombardo, S. J., and Bell, A. T., 1989. Monte-Carlo Simulation of Temperature-Programmed Desorption of Coadsorbed Species. Surface Science 224(1-3), 451-475.

Lou, Y., and Christofides, P. D., 2003. Feedback control of growth rate and surface roughness in thin film growth. AIChE Journal 49(8), 2099-2113.

Ludwig, J., and Vlachos, D. G., 2007. Ab initio molecular dynamics of hydrogen dissociation on metal surfaces using neural networks and novelty sampling. Journal of Chemical Physics 127(15), 154716.

Ludwig, J., and Vlachos, D. G., 2008. Molecular dynamics of hydrogen dissociation on an oxygen covered Pt(111) surface. Journal of Chemical Physics 128(15), -.

Lukkien, J. J., Segers, J. P. L., Hilbers, P. A. J., Gelten, R. J., and Jansen, A. P. J., 1998. Efficient Monte Carlo methods for the simulation of catalytic surface reactions. Physical Review E 58(2), 2598-2610.

Lukkien, J. J., CARLOS Project: a general purpose program for the simulation of chemical reactions taking place at crystal surfaces. 2009, Technische Universiteit Eindhoven.

Lutsevich, L. V., Elokhin, V. I., Myshlyavtsev, A. V., Usov, A. G., and Yablonskii, G. S., 1991. Monte-Carlo Modeling of a Simple Catalytic Reaction-Mechanism - Comparison with Langmuir Kinetics. Journal of Catalysis 132(2), 302-310.

Maestri, M., Vlachos, D. G., Beretta, A., Groppi, G., and Tronconi, E., 2008. Steam and dry reforming of methane on Rh: Microkinetic analysis and hierarchy of kinetic models. Journal of Catalysis 259(2), 211-222.

Maestri, M., Vlachos, D. G., Beretta, A., Groppi, G., and Rronconi, E., 2009. A C-1 Microkinetic Model for Methane Conversion to Syngas on Rh/A12O3. Aiche Journal 55(4), 993-1008.

Maestri, M., and Reuter, K., 2011. Semiempirical Rate Constants for Complex Chemical Kinetics: First-Principles Assessment and Rational Refinement. Angewandte Chemie International Edition 123, 1226-1229.

Makeev, A. G., Maroudas, D., Panagiotopoulos, A. Z., and Kevrekidis, I. G., 2002. Coarse bifurcation analysis of kinetic Monte Carlo simulations: A lattice-gas model with lateral interactions. Journal of Chemical Physics 117(18), 8229-8240. 
Martínez, E., Monasterio, P. R., and Marian, J., 2011. Billion-atom synchronous parallel kinetic Monte Carlo simulations of critical 3D Ising systems. Journal of Computational Physics 230(4), 1359-1369.

Masel, R. I., Principles of Adsorption and Reaction on Solid Surfaces. 1996, New York: John Wiley \& Sons, Inc.

Mayhall, N. J., Raghavachari, K., Redfern, P. C., and Curtiss, L. A., 2009. Investigation of Gaussian4 Theory for Transition Metal Thermochemistry. Journal of Physical Chemistry A 113(17), 5170-5175.

McQuarrie, D. A., Statistical Mechanics. Harper's Chemistry Series, ed. S.A. Rice. 1976, New York, NY: Harper and Row, Publishers, Inc. 641.

Meakin, P., and Scalapino, D. J., 1987. Simple-Models for Heterogeneous Catalysis - Phase Transition-Like Behavior in Nonequilibrium Systems. Journal of Chemical Physics 87(1), 731-741.

Mei, D., Sheth, P. A., Neurock, M., and Smith, C. M., 2006. First-principles-based kinetic Monte Carlo simulation of the selective hydrogenation of acetylene over $\operatorname{Pd}(111)$. Journal of Catalysis 242(1), 1-15.

Mei, D. H., Neurock, M., and Smith, C. M., 2009. Hydrogenation of acetylene-ethylene mixtures over Pd and Pd-Ag alloys: First-principles-based kinetic Monte Carlo simulations. Journal of Catalysis 268(2), 181-195.

Mei, D. H., Du, J. C., and Neurock, M., 2010. First-Principles-Based Kinetic Monte Carlo Simulation of Nitric Oxide Reduction over Platinum Nanoparticles under Lean-Burn Conditions. Industrial \& Engineering Chemistry Research 49(21), 10364-10373.

Meng, B. Q., Weinberg, W. H., and Evans, J. W., 1994. Lattice-Gas Model Mimicking the No+Co Reaction on Pt(100). Journal of Chemical Physics 101(4), 3234-3242.

Meskine, H., Matera, S., Scheffler, M., Reuter, K., and Metiu, H., 2009. Examination of the concept of degree of rate control by first-principles kinetic Monte Carlo simulations. Surface Science 603(10-12), 1724-1730.

Mhadeshwar, A. B., Wang, H., and Vlachos, D. G., 2003. Thermodynamic consistency in microkinetic development of surface reaction mechanisms. Journal of Physical Chemistry B 107(46), 12721-12733.

Mhadeshwar, A. B., Kitchin, J. R., Barteau, M. A., and Vlachos, D. G., 2004. The role of adsorbate-adsorbate interactions in the rate controlling step and the most abundant reaction intermediate of NH3 decomposition on Ru. Catalysis Letters 96(1-2), 13-22.

Mhadeshwar, A. B., and Vlachos, D. G., 2005a. Is the water-gas shift reaction on Pt simple? Computer-aided microkinetic model reduction, lumped rate expression, and rate-determining step. Catalysis Today 105(1), 162-172.

Mhadeshwar, A. B., and Vlachos, D. G., 2005b. Hierarchical, multiscale surface reaction mechanism development: $\mathrm{CO}$ and $\mathrm{H}_{2}$ oxidation, water-gas shift, and preferential oxidation of $\mathrm{CO}$ on Rh. Journal of Catalysis 234(1), 48-63.

Mhadeshwar, A. B., and Vlachos, D. G., 2005c. Hierarchical multiscale mechanism development for methane partial oxidation and reforming and for thermal decomposition of oxygenates on Rh. Journal of Physical Chemistry B 109(35), 16819-16835.

Mhadeshwar, A. B., and Vlachos, D. G., 2007. A catalytic reaction mechanism for methane partial oxidation at short contact times, reforming, and combustion, and for oxygenate decomposition and oxidation on platinum. Industrial \& Engineering Chemistry Research 46(16), 5310-5324. 
Mhadeshwar, A. B., Winkler, B. H., Eiteneer, B., and Hancu, D., 2009. Microkinetic modeling for hydrocarbon (HC)-based selective catalytic reduction (SCR) of NOx on a silver-based catalyst. Appl. Catal. B-Environ. 89(1-2), 229-238.

Michaelides, A., Liu, Z. P., Zhang, C. J., Alavi, A., King, D. A., and Hu, P., 2003. Identification of general linear relationships between activation energies and enthalpy changes for dissociation reactions at surfaces. J. Am. Chem. Soc. 125(13), 3704-3705.

Mortensen, J. J., Hammer, B., and Nørskov, J. K., 1998. A theoretical study of adsorbateadsorbate interactions on $\mathrm{Ru}(0001)$. Surface Science 414(3), 315-329.

Mpourmpakis, G., and Vlachos, D. G., 2009. The Effects of the MgO Support and Alkali Doping on the CO Interaction with Au. Journal of Physical Chemistry C 113, 7329-7335.

Mpourmpakis, G., Andriotis, A. N., and Vlachos, D. G., 2010a. Identification of Descriptors for the CO Interaction with Metal Nanoparticles. Nano Lett 10(3), 1041-1045.

Mpourmpakis, G., Stamatakis, M., Herrmann, S., Vlachos, D. G., and Andriotis, A. N., $2010 \mathrm{~b}$. Bridging the Materials Gap in Theoretical Catalysis. Phys. Rev. Lett., Submitted.

Myshlyavtsev, A. V., and Zhdanov, V. P., 1989. The Effect of Nearest-Neighbour and NextNearest-Neighbour Lateral Interactions on Thermal-Desorption Spectra. Chem Phys Lett 162(1-2), 43-46.

Neurock, M., and Hansen, E. W., 1998. First-principles-based molecular simulation of heterogeneous catalytic surface chemistry. Comput Chem Eng 22, S1045-S1060.

Nilekar, A. U., Greeley, J., and Mavrikakis, M., 2006. A simple rule of thumb for diffusion on transition-metal surfaces. Angewandte Chemie-International Edition 45(42), 7046-7049.

Nørskov, J. K., Bligaard, T., Logadottir, A., Bahn, S., Hansen, L. B., Bollinger, M., Bengaard, H., Hammer, B., Sljivancanin, Z., Mavrikakis, M., Xu, Y., Dahl, S., and Jacobsen, C. J. H., 2002. Universality in heterogeneous catalysis. Journal of Catalysis 209(2), 275-278.

Nørskov, J. K., Bligaard, T., Rossmeisl, J., and Christensen, C. H., 2009. Towards the computational design of solid catalysts. Nature Chemistry 1(1), 37-46.

Numaguchi, T., and Kikuchi, K., 1988. Intrinsic kinetics and design simulation in a complexreaction network - steam-methane reforming. Chem. Eng. Sci. 43(8), 2295-2301.

Oh, S. H., Fisher, G. B., Carpenter, J. E., and Goodman, D. W., 1986. Comparative kineticstudies of $\mathrm{CO}-\mathrm{O}_{2}$ and $\mathrm{CO}-\mathrm{NO}$ reactions over single-crystal and supported rhodium catalysts. Journal of Catalysis 100(2), 360-376.

Ovesson, S., Bogicevic, A., and Lundqvist, B. I., 1999. Origin of compact triangular islands in metal-on-metal growth. Phys. Rev. Lett. 83(13), 2608-2611.

Park, Y. K., Aghalayam, P., and Vlachos, D. G., 1999. A generalized approach for predicting coverage-dependent reaction parameters of complex surface reactions: Application to $\mathrm{H}_{2}$ oxidation over platinum. Journal of Physical Chemistry A 103(40), 8101-8107.

Pignedoli, C. A., Laino, T., Treier, M., Fasel, R., and Passerone, D., 2010. A simple approach for describing metal-supported cyclohexaphenylene dehydrogenation. European Physical Journal B 75(1), 65-70.

Prasad, V., Karim, A. M., Arya, A., and Vlachos, D. G., 2009. Assessment of Overall Rate Expressions and Multiscale, Microkinetic Model Uniqueness via Experimental Data Injection: Ammonia Decomposition on $\mathrm{Ru} /$ gamma-Al2O3 for Hydrogen Production. Industrial \& Engineering Chemistry Research 48(11), 5255-5265.

Qin, F., Tagliabue, L., Piovesan, L., and Wolf, E. E., 1998. Monte Carlo simulations of selfsustained oscillations of CO oxidation over non-isothermal supported catalysts. Chemical Engineering Science 53(5), 919-931. 
Quiceno, R., Perez-Ramirez, J., Warnatz, J., and Deutschmann, O., 2006. Modeling the hightemperature catalytic partial oxidation of methane over platinum gauze: Detailed gas-phase and surface chemistries coupled with 3D flow field simulations. Applied Catalysis a-General 303(2), 166-176.

Raghavachari, K., Stefanov, B. B., and Curtiss, L. A., 1997a. Accurate thermochemistry for larger molecules: Gaussian-2 theory with bond separation energies. Journal of Chemical Physics 106(16), 6764-6767.

Raghavachari, K., Stefanov, B. B., and Curtiss, L. A., 1997b. Accurate thermochemistry for medium-sized and large molecules. Abstracts of Papers of the American Chemical Society 213, 170-PHYS.

Raimondeau, S., and Vlachos, D. G., 2002. Recent developments on multiscale, hierarchical modeling of chemical reactors. Chemical Engineering Journal 90(1-2), 3-23.

Reuter, K., and Scheffler, M., 2002. Composition, structure, and stability of RuO2(110) as a function of oxygen pressure. Phys. Rev. B 65(3).

Reuter, K., Frenkel, D., and Scheffler, M., 2004. The steady state of heterogeneous catalysis, studied by first-principles statistical mechanics. Phys. Rev. Lett. 93(11), 116105.

Reuter, K., and Scheffler, M., 2006. First-principles kinetic Monte Carlo simulations for heterogeneous catalysis: Application to the $\mathrm{CO}$ oxidation at $\mathrm{RuO}_{2}(110)$. Phys. Rev. B 73(4), 045433.

Rieger, M., Rogal, J., and Reuter, K., 2008. Effect of surface nanostructure on temperature programmed reaction spectroscopy: First-principles kinetic Monte Carlo simulations of CO oxidation at $\mathrm{RuO}_{2}(110)$. Phys. Rev. Lett. 100(1), 016105.

Rogal, J., Reuter, K., and Scheffler, M., 2008. CO oxidation on $\operatorname{Pd(100)}$ at technologically relevant pressure conditions: First-principles kinetic Monte Carlo study. Phys. Rev. B 77(15), 155410.

Ruggerone, P., Ratsch, C., and Scheffler, M., "Density-functional theory of epitaxial growth of metals", in Growth and Properties of Ultrathin Epitaxial Layers, D.A. King and D.P. Woodruff, Editors. 1997, Elsevier Science: Amsterdam. p. 490-544.

Rusli, E., Drews, T. O., and Braatz, R. D., 2004. Systems analysis and design of dynamically coupled multiscale reactor simulation codes. Chemical Engineering Science 59, 5607-5613.

Salciccioli, M., Chen, Y., and Vlachos, D. G., 2010. Density Functional Theory-Derived Group Additivity and Linear Scaling Methods for Prediction of Oxygenate Stability on Metal Catalysts: Adsorption of Open-Ring Alcohol and Polyol Dehydrogenation Intermediates on Pt-Based Metals. The Journal of Physical Chemistry C 114(47), 20155-20166.

Salciccioli, M., Chen, Y., and Vlachos, D. G., 2011. Microkinetic Modeling and Reduced Rate Expressions of Ethylene Hydrogenation and Ethane Hydrogenolysis on Platinum. Industrial \& Engineering Chemistry Research 50(1), 28-40.

Salciccioli, M., Yu, W., Barteau, M. A., Chen, J. G., and Vlachos, D. G., In Press. Differentiation of O-H and C-H Bond Scission Mechanisms of Ethylene Glycol on Pt and $\mathrm{Ni} /$ Pt Using Theory and Isotopic Labeling Experiments.

Santiago, M. A. N., Sanchez-Castillo, M. A., Cortright, R. D., and Dumesic, J. A., 2000. Catalytic reduction of acetic acid, methyl acetate, and ethyl acetate over silica-supported copper. Journal of Catalysis 193(1), 16-28.

Schulze, T. P., Smereka, P., and E, W., 2003. Coupling kinetic Monte-Carlo and continuum models with application to epitaxial growth. Journal of Computational Physics 189(1), 197211. 
Schumacher, N., Boisen, A., Dahl, S., Gokhale, A. A., Kandoi, S., Grabow, L. C., Dumesic, J. A., Mavrikakis, M., and Chorkendorff, I., 2005. Trends in low-temperature water-gas shift reactivity on transition metals. Journal of Catalysis 229(2), 265-275.

Sheth, P. A., Neurock, M., and Smith, C. M., 2005. First-principles analysis of the effects of alloying $\mathrm{Pd}$ with $\mathrm{Ag}$ for the catalytic hydrogenation of acetylene-ethylene mixtures. Journal of Physical Chemistry B 109(25), 12449-12466.

Sholl, D. S., and Steckel, J. A., Density Functional Theory: A Practical Introduction. 1 ed. 2009, Hoboken, New Jersey: John Wiley \& Sons, Inc. 238.

Shustorovich, E., and Sellers, H., 1998. The UBI-QEP method: a practical theoretical approach to understanding chemistry on transition metal surfaces. Surface Science Reports 31(1-3), 5119.

Silverberg, M., Ben-Shaul, A., and Rebentrost, F., 1985. On the Effects of Adsorbate Aggregation on the Kinetics of Surface-Reactions. Journal of Chemical Physics 83(12), 6501-6513.

Silverberg, M., and Ben-Shaul, A., 1987a. Effects of Adsorbate Islanding on Reaction-Kinetics and Thermal-Desorption Spectra - a Monte-Carlo Quasi-Chemical Model. Chem Phys Lett 134(5), 491-496.

Silverberg, M., and Ben-Shaul, A., 1987b. Adsorbate Islanding in Surface-Reactions - a Combined Monte-Carlo-Lattice Gas Approach. Journal of Chemical Physics 87(5), 31783194.

Silverberg, M., and Ben-Shaul, A., 1989. Adsorbate Lateral Interactions and Islanding in Surface-Reaction Kinetics. Surface Science 214(1-2), 17-43.

Skoplyak, O., Barteau, M. A., and Chen, J. G., 2008a. Enhancing $\mathrm{H}_{2}$ and $\mathrm{CO}$ Production from Glycerol Using Bimetallic Surfaces. Chemsuschem 1(6), 524-526.

Skoplyak, O., Menning, C. A., Barteau, M. A., and Chen, J. G., 2008b. Reforming of Oxygenates for $\mathrm{H}_{2}$ Production on $3 \mathrm{~d} / \mathrm{Pt}(111)$ Bimetallic Surfaces. Topics in Catalysis 51(14), 49-59.

Stamatakis, M., and Vlachos, D. G., Under Review. A Novel Graph-Theoretical Kinetic Monte Carlo Framework for on-Lattice Chemical Kinetics. In preparation.

Stampfl, C., and Scheffler, M., 1996. Theoretical study of O adlayers on Ru(0001). Phys. Rev. B 54(4), 2868-2872.

Stiles, M., and Metiu, H., 1986. On the Interplay between Chemical-Reactions and PhaseTransitions for Molecules Adsorbed on Solid-Surfaces. Chem Phys Lett 128(4), 337-342.

Strasser, P., Fan, Q., Devenney, M., Weinberg, W. H., Liu, P., and Nørskov, J. K., 2003. High throughput experimental and theoretical predictive screening of materials - A comparative study of search strategies for new fuel cell anode catalysts. Journal of Physical Chemistry B 107(40), 11013-11021.

Tavazzi, I., Beretta, A., Groppi, G., and Forzatti, P., 2006. Development of a molecular kinetic scheme for methane partial oxidation over a $\mathrm{Rh} / \alpha-\mathrm{Al}_{2} \mathrm{O}_{3}$ catalyst Journal of Catalysis $241(1)$, $1-13$.

Temel, B., Meskine, H., Reuter, K., Scheffler, M., and Metiu, H., 2007. Does phenomenological kinetics provide an adequate description of heterogeneous catalytic reactions? Journal of Chemical Physics 126(20), 204711.

Trushin, O., Karim, A., Kara, A., and Rahman, T. S., 2005. Self-learning kinetic Monte Carlo method: Application to Cu(111). Phys. Rev. B 72(11), 115401. 
Ulissi, Z., Prasad, V., and Vlachos, D. G., 2010. Effect of multiscale model uncertainty on catalyst design. Journal of Catalysis, submitted.

van Santen, R. A., Neurock, M., and Shetty, S. G., 2010. Reactivity Theory of Transition-Metal Surfaces: A Bronsted-Evans-Polanyi Linear Activation Energy-Free-Energy Analysis. Chemical Reviews 110(4), 2005-2048.

Vlachos, D. G., 1997. Multiscale integration hybrid algorithms for homogeneous-heterogeneous reactors. AIChE Journal 43(11), 3031-3041.

Vlachos, D. G., 2005. A review of multiscale analysis: Examples from systems biology, materials engineering, and other fluid-surface interacting systems. Adv. Chem. Eng. 30, 1-61, invited.

Vlachos, D. G., Mhadeshwar, A. B., and Kaisare, N. S., 2006. Hierarchical multiscale modelbased design of experiments, catalysts, and reactors for fuel processing. Comput Chem Eng 30(10-12), 1712-1724.

Vlachos, D. G., and Caratzoulas, S., 2010. The roles of catalysis and reaction engineering in overcoming the energy and the environment crisis. Chem. Eng. Sci. 65, 18-29, Invited as part of the plenary lecture.

Voter, A. F., 1997a. Hyperdynamics: Accelerated molecular dynamics of infrequent events. Phys. Rev. Lett. 78(20), 3908-3911.

Voter, A. F., 1997b. A method for accelerating the molecular dynamics simulation of infrequent events. Journal of Chemical Physics 106(11), 4665-4677.

Wang, G. C., Cui, Y. B., Wang, Y. Y., Sun, Y. H., and Zhong, B., 1998. Microkinetic analysis of water gas shift reaction over $\mathrm{Cu}(110), \mathrm{Cu}(111)$ surfaces and Monte Carlo simulation of its mechanism. Chem J Chinese U 19(5), 779-782.

Wang, G. C., Tao, S. X., and Bu, X. H., 2006. A systematic theoretical study of water dissociation on clean and oxygen-preadsorbed transition metals. Journal of Catalysis 244(1), $10-16$.

Wang, H., and Brezinsky, K., 1998. Computational study on the thermochemistry of cyclopentadiene derivatives and kinetics of cyclopentadienone thermal decomposition. Journal of Physical Chemistry A 102(9), 1530-1541.

Wang, H., Stamatakis, M., Hansgen, D. A., Caratzoulas, S., and Vlachos, D. G., 2010. Understanding Mixing of $\mathrm{Ni}$ and $\mathrm{Pt}$ in the Ni/Pt(111) Bimetallic Catalyst via Molecular Simulation and Experiments. Journal of Chemical Physics 133, 224503.

Wang, H., Chen, Y., and Vlachos, D. G., 2011. On the Structure Sensitivity of Catalytic Reactions.

Wang, H. F., and Liu, Z. P., 2008. Comprehensive mechanism and structure-sensitivity of ethanol oxidation on platinum: New transition-state searching method for resolving the complex reaction network. J. Am. Chem. Soc. 130(33), 10996-11004.

Wei, J. M., and Iglesia, E., 2004. Isotopic and kinetic assessment of the mechanism of reactions of $\mathrm{CH} 4$ with $\mathrm{CO} 2$ or $\mathrm{H} 2 \mathrm{O}$ to form synthesis gas and carbon on nickel catalysts. Journal of Catalysis 224(2), 370-383.

Xu, J. G., and Froment, G. F., 1989. Methane Steam Reforming, Methanation and Water-Gas Shift .1. Intrinsic Kinetics. AIChE Journal 35(1), 88-96.

Yeo, Y. Y., Vattuone, L., and King, D. A., 1997. Calorimetric heats for CO and oxygen adsorption and for the catalytic $\mathrm{CO}$ oxidation reaction on $\operatorname{Pt}(111)$. Journal of Chemical Physics 106(1), 392-401. 
Zhdanov, V. P., and Kasemo, B., 1994. Kinetic Phase-Transitions in Simple Reactions on SolidSurfaces. Surface Science Reports 20(3), 113-189.

Zhdanov, V. P., and Kasemo, B., 1998. Monte Carlo simulation of the kinetics of rapid reactions on nanometer catalyst particles. Surface Science 405(1), 27-37.

Zhdanov, V. P., and Kasemo, B., 2000. Simulations of the reaction kinetics on nanometer supported catalyst particles. Surface Science Reports 39(2-4), 29-104.

Zhdanov, V. P., 2002. Monte Carlo simulations of oscillations, chaos and pattern formation in heterogeneous catalytic reactions. Surface Science Reports 45(7-8), 233-326.

Zhdanov, V. P., 2003. Electrochemical reactions on catalyst particles with three-phase boundaries. Physical Review E 67(4), 042601.

Ziff, R. M., Gulari, E., and Barshad, Y., 1986. Kinetic Phase Transitions in an Irreversible Surface-Reaction Model. Phys. Rev. Lett. 56(24), 2553-2556. 
Table 1. Overall reactions used to describe methane steam reforming. The reactions are catalytic combustion, steam reforming, water-gas shift and reforming combined with water-gas shift reaction. Kinetic rate expressions from (Xu and Froment, 1989) and (Numaguchi and Kikuchi, 1988).

\begin{tabular}{|c|c|c|}
\hline Reaction & Rate equation (Xu and Froment, 1989) & $\begin{array}{l}\text { Rate equation (Numaguchi and } \\
\text { Kikuchi, 1988) }\end{array}$ \\
\hline $\mathrm{CH}_{4}+2 \mathrm{O}_{2} \rightarrow \mathrm{CO}_{2}+2 \mathrm{H}_{2} \mathrm{O}$ & $r_{1}=\frac{k_{1 a} p_{C_{4}} p_{O_{2}}}{\left(1+K_{C H_{4}}^{o x} p_{C_{4}}+K_{O_{2}}^{o x} p_{O_{2}}\right)^{2}}+\frac{k_{1 b} p_{C_{4}} p_{O_{2}}}{\left(1+K_{C H_{4}}^{o x} p_{C_{4}}+K_{O_{2}}^{o x} p_{O_{2}}\right)}$ & \\
\hline $\mathrm{CH}_{4}+\mathrm{H}_{2} \mathrm{O} \leftrightarrow \mathrm{CO}+3 \mathrm{H}_{2}$ & $r_{2}=\frac{k_{2}^{X u} / p_{H_{2}}^{2.5}\left(p_{C_{4}} p_{H_{2} O}-p_{H_{2}}^{3} p_{C O} / K_{e q, 2}\right)}{\left(1+K_{C O} p_{C O}+K_{H_{2}} p_{H_{2}}+K_{C_{4}} p_{C_{4}}+K_{H_{2} O} p_{H_{2} O} / p_{H_{2}}\right)^{2}}$ & $r_{2}=\frac{k_{2}\left(p_{C_{H_{4}}}-p_{C_{H_{4}}}^{E q}\right)}{\left(p_{C H_{4}}^{\alpha_{R}} p_{H_{2} \mathrm{O}}^{\delta_{R}}\right)}$ \\
\hline $\mathrm{CO}+\mathrm{H}_{2} \mathrm{O} \leftrightarrow \mathrm{CO}_{2}+\mathrm{H}_{2}$ & $r_{3}=\frac{k_{3}^{X u} / p_{\mathrm{H}_{2}}\left(p_{\mathrm{CO}} p_{\mathrm{H}_{2} \mathrm{O}}-p_{\mathrm{H}_{2}} p_{\mathrm{CO}_{2}} / K_{e q, 3}\right)}{\left(1+K_{\mathrm{CO}} p_{\mathrm{CO}}+K_{\mathrm{H}_{2}} p_{\mathrm{H}_{2}}+K_{\mathrm{CH}_{4}} p_{\mathrm{CH}_{4}}+K_{\mathrm{H}_{2} \mathrm{O}} p_{\mathrm{H}_{2} \mathrm{O}} / p_{\mathrm{H}_{2}}\right)^{2}}$ & $r_{3}=\frac{k_{3}\left(p_{C O}-p_{C O}^{E q}\right)}{\left(p_{C H_{4}}^{\alpha_{S}} p_{H_{2} O}^{\delta_{S}}\right)}$ \\
\hline $\mathrm{CH}_{4}+2 \mathrm{H}_{2} \mathrm{O} \leftrightarrow \mathrm{CO}_{2}+4 \mathrm{H}_{2}$ & $r_{4}=\frac{k_{4}^{X u} / p_{H_{2}}^{3.5}\left(p_{\mathrm{CH}_{4}} p_{\mathrm{H}_{2} \mathrm{O}}^{2}-p_{\mathrm{H}_{2}}^{4} p_{\mathrm{CO}_{2}} / K_{e q, 4}\right)}{\left(1+K_{\mathrm{CO}} p_{\mathrm{CO}}+K_{\mathrm{H}_{2}} p_{\mathrm{H}_{2}}+K_{\mathrm{CH}_{4}} p_{\mathrm{CH}_{4}}+K_{\mathrm{H}_{2} \mathrm{O}} p_{\mathrm{H}_{2} \mathrm{O}} / p_{\mathrm{H}_{2}}\right)^{2}}$ & \\
\hline
\end{tabular}


Table 2. Comparison of estimated from fitting and literature (either DFT or experimental) values for heats of adsorption. Since the coverage of $\mathrm{H}_{2} \mathrm{O}$ and $\mathrm{OH}$ on $\mathrm{Rh}$ under typical conditions is low, the fitted heat of adsorption of $\mathrm{H}_{2} \mathrm{O}$ (second column) differs considerably from that of DFT and experiment (third column). Similarly, even if the coverage of $\mathrm{CO}$ on $\mathrm{Rh}$ is high, there is significant discrepancy between fitted (second column) and experimental and DFT values (third column).

\begin{tabular}{|c|c|c|}
\hline $\begin{array}{l}\text { Surface } \\
\text { species }\end{array}$ & $\begin{array}{l}\text { Heat of adsorption (Q) } \\
\text { from fitting }[\mathrm{kcal} / \mathrm{mol}] ; \\
\text { from (Tavazzi et al., } \\
2006)\end{array}$ & $\begin{array}{c}\text { Heat of adsorption (Q) from } \\
\text { experiments or DFT [kcal/mol]; } \\
\text { see (Mhadeshwar and Vlachos, } \\
2005 \mathrm{~b})\end{array}$ \\
\hline $\mathrm{H}_{2} \mathrm{O}$ & -39.5 & $-10.8+4.5 \theta_{\mathrm{H} 2 \mathrm{O}}-25 \theta_{\mathrm{OH}}$ \\
\hline $\mathrm{CO}$ & -6.2 & $-38.5+17 \theta_{\mathrm{CO}}+3.7 \theta_{\mathrm{H}}$ \\
\hline
\end{tabular}


Table 3. Examples of microkinetic models in recent literature.

\begin{tabular}{|c|c|c|c|}
\hline Application & $\begin{array}{l}\text { Model } \\
\text { Type/Method (see } \\
\text { Figure 1) }\end{array}$ & Model Description & Reference \\
\hline \multirow{5}{*}{$\begin{array}{c}\text { Fundamental } \\
\text { mechanistic insights }\end{array}$} & DFT & $\begin{array}{l}\text { DFT-based NO oxidation on } \mathrm{Pt} \text { (emphasis on coverage } \\
\text { dependent model parameters) }\end{array}$ & $\begin{array}{l}\text { (Getman and } \\
\text { Schneider, 2010) }\end{array}$ \\
\hline & DFT & $\begin{array}{l}\text { DFT-based } \mathrm{CH}_{4} \text { steam reforming on } \mathrm{Ni} \text { (thorough } \\
\text { implementation of statistical mechanics for temperature } \\
\text { dependent kinetic parameters) }\end{array}$ & $\begin{array}{l}\text { (Blaylock et al., } \\
\text { 2009) }\end{array}$ \\
\hline & DFT & DFT-based water-gas shift mechanism on Pt & (Grabow et al., 2008) \\
\hline & DFT and BOC & $\begin{array}{l}\mathrm{NH}_{3} \text { decomposition on } \mathrm{Ru} \text { (applying DFT calculated } \\
\text { coverage dependent atomic binding energies into semi- } \\
\text { empirical methods) }\end{array}$ & $\begin{array}{l}\text { (Mhadeshwar et al., } \\
\text { 2004) }\end{array}$ \\
\hline & $\mathrm{BOC}$ & $\begin{array}{l}\text { Hydrocarbon-based selective catalytic reduction of } \mathrm{NO}_{\mathrm{x}} \\
\text { on } \mathrm{Ag}\end{array}$ & $\begin{array}{l}\text { (Mhadeshwar et al., } \\
\text { 2009) }\end{array}$ \\
\hline \multirow{5}{*}{ Catalyst Design } & $\begin{array}{l}\text { DFT, linear scaling } \\
\text { and BEP }\end{array}$ & $\begin{array}{l}\text { BEP and linear scaling relationships. } \mathrm{CH}_{4} \text { steam } \\
\text { reforming activity across transition metals }\end{array}$ & (Jones et al., 2008b) \\
\hline & DFT and BOC & $\begin{array}{l}\mathrm{NH}_{3} \text { decomposition on several metals (uses model to find } \\
\text { optimal } \mathrm{N} \text { binding energy) }\end{array}$ & $\begin{array}{l}\text { (Hansgen et al., } \\
2010)\end{array}$ \\
\hline & DFT & $\begin{array}{l}\text { Ethanol synthesis from syngas on } \mathrm{Rh} \text { (sensitivity analysis } \\
\text { is used to probe for catalyst promoters) }\end{array}$ & (Choi and Liu, 2009) \\
\hline & DFT and BEP & $\begin{array}{l}\text { CO oxidation activity of nanoparticles (simplified model } \\
\text { to explain activity of gold nanoparticles at low } \\
\text { temperatures and pressures) }\end{array}$ & (Falsig et al., 2008) \\
\hline & $\begin{array}{l}\text { DFT, linear scaling } \\
\text { and BEP }\end{array}$ & $\begin{array}{l}\text { Water-gas shift model with DFT derived linear free } \\
\text { energy relationships to probe the } \mathrm{CO} \text { and } \mathrm{O} \text { binding } \\
\text { energy phase space for active catalysts }\end{array}$ & $\begin{array}{l}\text { (Schumacher et al., } \\
\text { 2005) }\end{array}$ \\
\hline \multirow[t]{2}{*}{ Reactor Design } & $\begin{array}{l}\text { BOC and } \\
\text { experimental tuning }\end{array}$ & $\begin{array}{l}\mathrm{CH}_{4} \text { reforming chemistries on } \mathrm{Rh} \text { (multiscale refinement } \\
\text { and adjustments to predict experimental results; derived } \\
\text { reduced rate expression) }\end{array}$ & (Maestri et al., 2008) \\
\hline & Experimental tuning & $\begin{array}{l}\mathrm{CH}_{4} \text { partial oxidation over Pt microkinetic model inserted } \\
\text { into CFD model }\end{array}$ & $\begin{array}{l}\text { (Quiceno } \\
2006)\end{array}$ \\
\hline
\end{tabular}


Table 4. Arrhenius parameters for a subset of the water-gas shift chemistry.

\begin{tabular}{|c|c|c|c|c|}
\hline Elementary Reaction & $\begin{array}{l}\text { Pre-exponential } \\
\text { factor }[1 / \mathrm{s}]\end{array}$ & $\beta$ & $\begin{array}{c}\Delta \mathrm{E}_{\mathrm{i}} \\
{[\mathrm{kcal} / \mathrm{mol}]}\end{array}$ & $\begin{array}{c}\Delta \mathrm{E}_{\mathrm{i}, \mathrm{rxn}}^{\mathrm{b}} \\
{[\mathrm{kcal} / \mathrm{mol}]}\end{array}$ \\
\hline $\mathrm{CO}+* \rightarrow \mathrm{CO}^{*}$ & $1.00^{\mathrm{a}}$ & 0.000 & 0.0 & -42.0 \\
\hline $\mathrm{CO}^{*} \rightarrow \mathrm{CO}+*$ & $5.66 \times 10^{15}$ & -0.500 & 40.0 & \\
\hline $\mathrm{H}_{2} \mathrm{O}+* \rightarrow \mathrm{H}_{2} \mathrm{O}^{*}$ & $0.108^{\mathrm{a}}$ & 1.162 & 0.0 & -6.2 \\
\hline $\mathrm{H}_{2} \mathrm{O}^{*} \rightarrow \mathrm{H}_{2} \mathrm{O}+*$ & $2.03 \times 10^{12}$ & 1.372 & 10.0 & \\
\hline $\mathrm{H}_{2} \mathrm{O}^{*}+* \rightarrow \mathrm{H}^{*}+\mathrm{OH}^{*}$ & $9.36 \times 10^{12}$ & -0.118 & 17.8 & 16.1 \\
\hline $\mathrm{H}^{*}+\mathrm{OH}^{*} \rightarrow \mathrm{H}_{2} \mathrm{O}^{*}+*$ & $9.99 \times 10^{12}$ & -1.049 & 13.5 & \\
\hline $\mathrm{CO}^{*}+\mathrm{OH}^{*} \rightarrow \mathrm{COOH}^{*}+*$ & $1.19 \times 10^{9}$ & -0.024 & 19.1 & -10.4 \\
\hline $\mathrm{COOH}^{*}+* \rightarrow \mathrm{CO}^{*}+\mathrm{OH}^{*}$ & $8.43 \times 10^{8}$ & 0.024 & 5.3 & \\
\hline $\mathrm{COOH}^{*}+* \rightarrow \mathrm{CO}_{2}^{*}+\mathrm{H}^{*}$ & $1.06 \times 10^{11}$ & 0.549 & 1.0 & -0.2 \\
\hline $\mathrm{CO}_{2} *+\mathrm{H}^{*} \rightarrow \mathrm{COOH}^{*}+*$ & $9.45 \times 10^{10}$ & -0.549 & 2.4 & \\
\hline $2 \mathrm{H}^{*} \rightarrow \mathrm{H}_{2}+2^{*}$ & $7.95 \times 10^{12}$ & -0.001 & 19.8 & 19.6 \\
\hline $\mathrm{H} 2+2 * \rightarrow 2 \mathrm{H}^{*}$ & $0.129^{\mathrm{a}}$ & 0.858 & 0.0 & \\
\hline $\mathrm{CO}_{2} * \rightarrow \mathrm{CO}_{2}+*$ & $3.63 \times 10^{12}$ & -0.250 & 3.6 & 2.5 \\
\hline $\mathrm{CO}_{2}+* \rightarrow \mathrm{CO}_{2}^{*}$ & $0.195^{\mathrm{a}}$ & 0.250 & 0.0 & \\
\hline
\end{tabular}

All Arrhenius values taken from (Mhadeshwar and Vlachos, 2007). ${ }^{\mathrm{a}}$ Value shown is a sticking coefficient (unitless). ${ }^{\mathrm{b}}$ Values for $\Delta \mathrm{E}$ are DFT numbers taken from (Grabow et al., 2008) (not corrected for ZPE). 
Table 5. Adjustment in activation energy based on Eq. 12 for competitive dehydrogenation reactions of ethanol on $\operatorname{Pd}(111)$.

\begin{tabular}{ccc}
\hline Parameter & & \\
{$[\mathrm{kcal} / \mathrm{mol}]$} & $\mathrm{CH}_{3} \mathrm{CH}_{2} \mathrm{OH}^{*} \rightarrow \mathrm{CH}_{3} \mathrm{CHOH}^{*}+\mathrm{H}^{*}$ & $\mathrm{CH}_{3} \mathrm{CH}_{2} \mathrm{O}^{*} \rightarrow \mathrm{CH}_{2} \mathrm{CH}_{2} \mathrm{OH}^{*}+\mathrm{H}^{*}$ \\
\hline$\Delta \mathrm{H}_{\mathrm{i} \text {,surf }}$ & 2.1 & 3.6 \\
$\Delta \mathrm{E}_{\mathrm{i} \text {,rxn,DFT }}$ & 2.3 & 11.0 \\
$\Delta \mathrm{E}_{\mathrm{i}, \mathrm{DFT}}$ & 21.3 & 21.9 \\
$\Delta \mathrm{E}_{\mathrm{i}}$ & 21.1 & 16.4 \\
\hline
\end{tabular}

DFT data taken from (Li et al., 2009). Gas-phase thermochemical data taken from (Dyke et al., 1997). For this example $\omega$ is set equal to 0.75 reflecting a moderately late transition state. 
Table 6. Estimates of pre-exponential factors for LHHW reactions.

Reaction type: $\mathrm{A}^{*}+\mathrm{B}^{*} \rightarrow \mathrm{C}^{*}+\mathrm{D}^{*} \quad$ factor estimate $[1 / \mathrm{s}]$

Pre-exponential

Mobile surface species with rotation $10^{8}$

Mobile surface species without rotation $\quad 10^{11}$

Immobile surface species without rotation $10^{13}$

Information taken from (Dumesic et al., 1993). 
Table 7. Full C2 mechanism for ethane hydrogenolysis and ethylene hydrogenation.

\begin{tabular}{|c|c|}
\hline $\begin{array}{l}\text { Reaction } \\
\text { Index }\end{array}$ & Reaction \\
\hline $\mathrm{R} 1$ & $\mathrm{H}_{2}+2^{*} \leftrightarrow 2 \mathrm{H}^{*}$ \\
\hline R2 & $\mathrm{CH}_{4}+2^{*} \leftrightarrow \mathrm{CH}_{3}^{*}+\mathrm{H}^{*}$ \\
\hline R3 & $\mathrm{C}_{2} \mathrm{H}_{4}+2 * \leftrightarrow \pi-\mathrm{C}_{2} \mathrm{H}_{4} * *$ \\
\hline R4 & $\mathrm{C}_{2} \mathrm{H}_{4}+4 * \leftrightarrow \sigma-\mathrm{C}_{2} \mathrm{H}_{4} * * * *$ \\
\hline R5 & $\mathrm{C}_{2} \mathrm{H}_{2}+2 * \leftrightarrow \mathrm{C}_{2} \mathrm{H}_{2} * *$ \\
\hline R6 & $\mathrm{C}_{2} \mathrm{H}_{6}+3 * \leftrightarrow \mathrm{C}_{2} \mathrm{H}_{5} * *+\mathrm{H}^{*}$ \\
\hline R7 & $\pi-\mathrm{C}_{2} \mathrm{H}_{4} * *+2 * \leftrightarrow \sigma-\mathrm{C}_{2} \mathrm{H}_{4} * * * *$ \\
\hline R8 & $\pi-\mathrm{C}_{2} \mathrm{H}_{4} * *+\mathrm{H}^{*} \leftrightarrow \mathrm{C}_{2} \mathrm{H}_{5}^{* *}+*$ \\
\hline R9 & $\mathrm{C}_{2} \mathrm{H}_{5}^{* *}+3^{*} \leftrightarrow \sigma-\mathrm{C}_{2} \mathrm{H}_{4} * * * *+\mathrm{H}^{*}$ \\
\hline R10 & $\sigma-\mathrm{C}_{2} \mathrm{H}_{4} * * * * \leftrightarrow \mathrm{C}_{2} \mathrm{H}_{3} * *+\mathrm{H}^{*}+*$ \\
\hline R11 & $\mathrm{C}_{2} \mathrm{H}_{2} * *+\mathrm{H}^{*} \leftrightarrow \mathrm{C}_{2} \mathrm{H}_{3} * *+*$ \\
\hline R12 & $\mathrm{C}_{2} \mathrm{H}^{* *}+\mathrm{H}^{*} \leftrightarrow \mathrm{C}_{2} \mathrm{H}_{2} * *+*$ \\
\hline R13 & $\mathrm{CHCH}_{3} * * \leftrightarrow \mathrm{CCH}_{3} *+\mathrm{H}^{*}$ \\
\hline R14 & $\mathrm{CCH}_{3} *+2 * \leftrightarrow \mathrm{CCH}_{2} * *+\mathrm{H}^{*}$ \\
\hline R15 & $\mathrm{C}_{2} \mathrm{H}_{5} * *+* \leftrightarrow \mathrm{CHCH}_{3} * *+\mathrm{H}^{*}$ \\
\hline R16 & $\mathrm{CHCH}_{3} * *+* \leftrightarrow \mathrm{C}_{2} \mathrm{H}_{3} * *+\mathrm{H}^{*}$ \\
\hline R17 & $\mathrm{C}_{2} \mathrm{H}_{3} * *+* \leftrightarrow \mathrm{CCH}_{2} * *+\mathrm{H}^{*}$ \\
\hline R18 & $\mathrm{C}_{2} \mathrm{H}^{* *}+\mathrm{H}^{*} \leftrightarrow \mathrm{CCH}_{2} * *+*$ \\
\hline R19 & $\mathrm{C}_{2} \mathrm{H}_{5} * * \leftrightarrow \mathrm{CH}_{3} *+\mathrm{CH}_{2} *$ \\
\hline R20 & $2 \mathrm{CH}_{2} *+2 * \leftrightarrow \sigma-\mathrm{C}_{2} \mathrm{H}_{4} * * * *$ \\
\hline R21 & $\mathrm{C}_{2} \mathrm{H}_{3} * * \leftrightarrow \mathrm{CH}^{*}+\mathrm{CH}_{2} *$ \\
\hline R22 & $\mathrm{C}_{2} \mathrm{H}_{2} * * \leftrightarrow 2 \mathrm{CH}^{*}$ \\
\hline R23 & $\mathrm{C}_{2} \mathrm{H}^{* *} \leftrightarrow \mathrm{CH}^{*}+\mathrm{C}^{*}$ \\
\hline R24 & $\mathrm{CHCH}_{3} * * \leftrightarrow \mathrm{CH}_{3} *+\mathrm{CH}^{*}$ \\
\hline R25 & $\mathrm{CH}_{3} *+\mathrm{C}^{*} \leftrightarrow \mathrm{CCH}_{3} *+*$ \\
\hline R26 & $\mathrm{CCH}_{2} * * \leftrightarrow \mathrm{CH}_{2} *+\mathrm{C}^{*}$ \\
\hline R27 & $\mathrm{CHCH}_{3} * *+2 * \leftrightarrow \mathrm{C}_{2} \mathrm{H}_{4} * * * *$ \\
\hline R28 & $\mathrm{C}_{2} \mathrm{H}_{3} * * \leftrightarrow \mathrm{CCH}_{3} *+*$ \\
\hline R29 & $\mathrm{C}_{2} \mathrm{H}_{2} * * \leftrightarrow \mathrm{CCH}_{2} * *$ \\
\hline R30 & $\mathrm{C}^{*}+\mathrm{H}^{*} \leftrightarrow \mathrm{CH}^{*}+*$ \\
\hline R31 & $\mathrm{CH}_{2} *+* \leftrightarrow \mathrm{CH}^{*}+\mathrm{H}^{*}$ \\
\hline R32 & $\mathrm{CH}_{3} *+* \leftrightarrow \mathrm{CH}_{2}^{*}+\mathrm{H}^{*}$ \\
\hline
\end{tabular}


Table 8. Library of DFT binding energies (BEs) of atomic nitrogen (N) at a 1/9th monolayer (ML) coverage on various monolayer bimetallic surfaces on a $\mathrm{Pt}(111)$ host. The $\mathrm{BE}$ of $\mathrm{N}$ on bimetallic surfaces varies widely. The different colors in the structures (left column) indicate different architectures. Taken from (Hansgen et al., 2010).

\begin{tabular}{llc}
\hline Structure & $(111)$ & $\begin{array}{c}\mathrm{Q}_{\mathrm{N}} \\
(\mathrm{kcal} / \mathrm{mol})\end{array}$ \\
\hline & Surface & -70.6 \\
Subsurface & Pt-Ti-Pt & -80.9 \\
& Pt-V-Pt & -76.3 \\
& Pt-Cr-Pt & -77.5 \\
& Pt-Mn-Pt & -78.4 \\
& Pt-Fe-Pt & -83.5 \\
Single Metal & Pt-Co-Pt & -87.4 \\
& Pt-Ni-Pt & -102.2 \\
Top & Pt & -113.7 \\
& Ni & -130.8 \\
& Ni-Pt-Pt & -126.4 \\
& Co-Pt-Pt & -134.2 \\
& Fe-Pt-Pt & -207.1 \\
& Mn-Pt-Pt & -188.4 \\
& Cr-Pt-Pt & -188.2 \\
& V-Pt-Pt & -176.2 \\
\hline
\end{tabular}


Table 9. Au surfaces simulated with the kinetic Monte Carlo method.

\section{Surface Step:Total \\ Sites Ratio}

\begin{tabular}{lc}
\hline $\mathrm{Au}(111)$ & 0 \\
$\mathrm{Au}(544)$ & $1 / 9$ \\
$\mathrm{Au}(755)$ & $1 / 6$ \\
$\mathrm{Au}(322)$ & $1 / 5$ \\
$\mathrm{Au}(533)$ & $1 / 4$ \\
$\mathrm{Au}(211)$ & $1 / 3$ \\
\hline
\end{tabular}


Table 10a. Elementary steps and kinetic parameters for terrace (trc) site events for the CO oxidation on Au used in the microkinetic model simulated with the kinetic Monte Carlo method.

\begin{tabular}{cccc}
\hline Element Step & $\begin{array}{c}\text { Prefactor A } \\
(\mathrm{fwd}, \mathrm{bkwd})\end{array}$ & Activation Energy $(\mathrm{eV})$ & Reaction energy $(\mathrm{eV})$ \\
$\mathrm{O}_{2}(\mathrm{~g})+* \rightarrow \mathrm{O}_{2}^{*}$ & $1.8 \cdot 10^{8} \mathrm{bar}^{-1}, 8.6 \cdot 10^{14}$ & 0 & $0.89 \Delta \mathrm{E}_{\mathrm{O}, \text { trc }}+0.17$ \\
$\mathrm{CO}(\mathrm{g})+* \rightarrow \mathrm{CO}^{*}$ & $1.9 \cdot 10^{8} \mathrm{bar}^{-1}, 8.6 \cdot 10^{14}$ & 0 & $\Delta \mathrm{E}_{\mathrm{CO}, \mathrm{trc}}$ \\
$\mathrm{O}_{2}{ }^{*} \rightarrow 2 \mathrm{O}^{*}$ & $6.2 \cdot 10^{12}, 3.3 \cdot 10^{12}$ & $0.50 \Delta \mathrm{E}_{\mathrm{O}, \mathrm{trc}}+1.39$ & $1.11 \Delta \mathrm{E}_{\mathrm{O}, \mathrm{rrc}}-0.17$ \\
$\mathrm{CO}^{*}+\mathrm{O}^{*} \rightarrow$ & $1.1 \cdot 10^{13}, 8.2 \cdot 10^{12} \mathrm{bar}^{-1}$ & $-0.3\left(\Delta \mathrm{E}_{\mathrm{O}, \mathrm{trc}}+\Delta \mathrm{E}_{\mathrm{CO}, \text { trc }}\right)+$ & $-3.08-\Delta \mathrm{E}_{\mathrm{O}, \mathrm{trc}}-$ \\
$\mathrm{CO}_{2}(\mathrm{~g})+2^{*}$ & & 0.02 & $\Delta \mathrm{E}_{\mathrm{CO}, \mathrm{trc}}$ \\
\hline
\end{tabular}

Coverage effects: $\Delta \mathrm{E}_{\mathrm{O}, \mathrm{trc}}=-0.2+2.76 \theta_{\mathrm{CO}}+2.83\left(\theta_{\mathrm{O} 2}+\theta_{\mathrm{O}}\right) ; \Delta \mathrm{E}_{\mathrm{CO}, \mathrm{trc}}=0.12+1.312 \theta_{\mathrm{CO}}+$ $2.011\left(\theta_{\mathrm{O} 2}+\theta_{\mathrm{O}}\right)$

Table 10b. Elementary steps and kinetic parameters for step (stp) site events.

\begin{tabular}{cccc}
\hline Reaction Step & $\begin{array}{c}\text { Pre-factor A } \\
(\mathrm{fwd}, \text { bkwd })\end{array}$ & Activation energy, (eV) & Reaction energy (eV) \\
\hline $\mathrm{O}_{2}(\mathrm{~g})+* \rightarrow \mathrm{O}_{2}{ }^{*}$ & $1.8 \cdot 10^{8} \mathrm{bar}^{-1}, 8.6 \cdot 10^{14}$ & 0 & $0.89 \Delta \mathrm{E}_{\mathrm{O}, \text { step }}-0.3$ \\
$\mathrm{CO}(\mathrm{g})+* \rightarrow \mathrm{CO}^{*}$ & $1.9 \cdot 10^{8} \mathrm{bar}^{-1}, 8.6 \cdot 10^{14}$ & 0 & $\Delta \mathrm{E}_{\mathrm{CO}, \text { step }}$ \\
$\mathrm{O}_{2}{ }^{*} \rightarrow 2 \mathrm{O}^{*}$ & $7.4 \cdot 10^{12}, 4.2 \cdot 10^{12}$ & $0.50 \Delta \mathrm{E}_{\mathrm{O}, \text { step }}+0.82$ & $1.11 \Delta \mathrm{E}_{\mathrm{O}, \text { step }}+0.3$ \\
$\mathrm{CO}^{*}+\mathrm{O}^{*} \rightarrow \mathrm{CO}_{2}(\mathrm{~g})+2^{*}$ & $5.6 \cdot 10^{13}, 1.3 \cdot 10^{13} \mathrm{bar}^{-1}$ & $-0.3\left(\Delta \mathrm{E}_{\mathrm{O}, \text { step }}+\Delta \mathrm{E}_{\mathrm{CO}, \text { step }}\right)+0.02$ & $-3.08-\Delta \mathrm{E}_{\mathrm{O}, \text { step }}-\Delta \mathrm{E}_{\mathrm{CO}, \text { step }}$ \\
$\mathrm{CO}^{*}+\mathrm{O}_{2}{ }^{*} \rightarrow$ & $8.2 \cdot 10^{13}, 2.9 \cdot 10^{13} \mathrm{bar}^{-1}$ & $-0.11 \Delta \mathrm{E}_{\mathrm{O}, \text { step }}-0.22 \Delta \mathrm{E}_{\mathrm{CO} \text {,step }}+$ & $-2.69+0.11 \Delta \mathrm{E}_{\mathrm{O}, \text { step }}-\Delta \mathrm{E}_{\mathrm{CO}, \text { step }}$ \\
$\mathrm{CO}_{2}(\mathrm{~g})+\mathrm{O}^{*}+$ & & 0.092 & \\
\hline
\end{tabular}

Coverage effects: $\Delta \mathrm{E}_{\mathrm{O}, \mathrm{stp}}=-0.56+2.76 \theta_{\mathrm{CO}}+2.83\left(\theta_{\mathrm{O} 2}+\theta_{\mathrm{O}}\right) ; \Delta \mathrm{E}_{\mathrm{CO}, \mathrm{stp}}=-0.54+1.312 \theta_{\mathrm{CO}}+$ $2.011\left(\theta_{\mathrm{O} 2}+\theta_{\mathrm{O}}\right)$ 


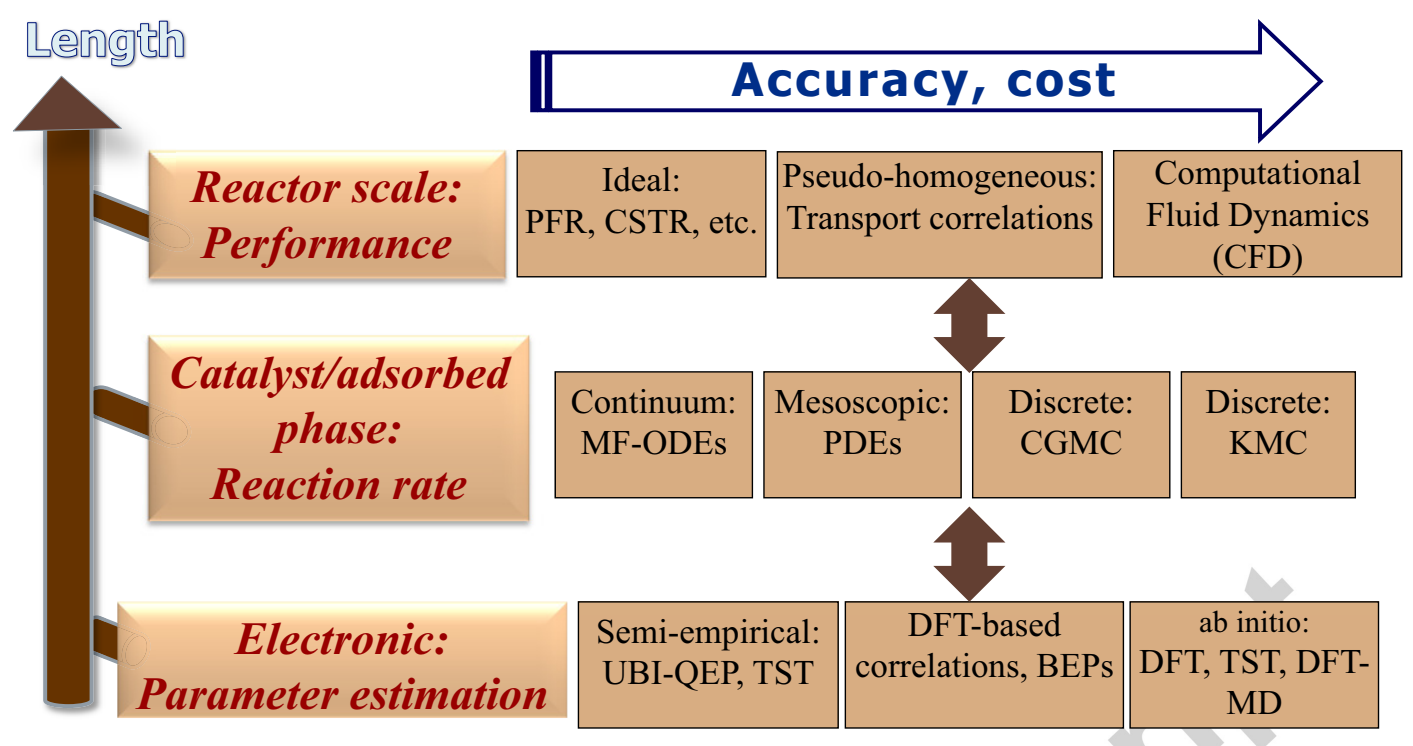

Figure 1. Schematic of three scales and a possible hierarchy of models at each scale. At each scale, additional models may exist. The accuracy and cost increase from left to right. Acronyms from top to bottom: PRF, plug flow reactor; CSTR, continuously stirred tank reactor; ODE, ordinary differential equation; PDE, partial differential equation; CG-KMC, coarse-grained kinetic Monte Carlo; KMC, kinetic Monte Carlo; UBI-QEP, unity bond indexquadratic exponential potential; TST, transition state theory; DFT, density functional theory; GA, group additivity; BEP, Brønsted-Evans-Polanyi; QM/MM, quantum mechanics/molecular mechanics. 


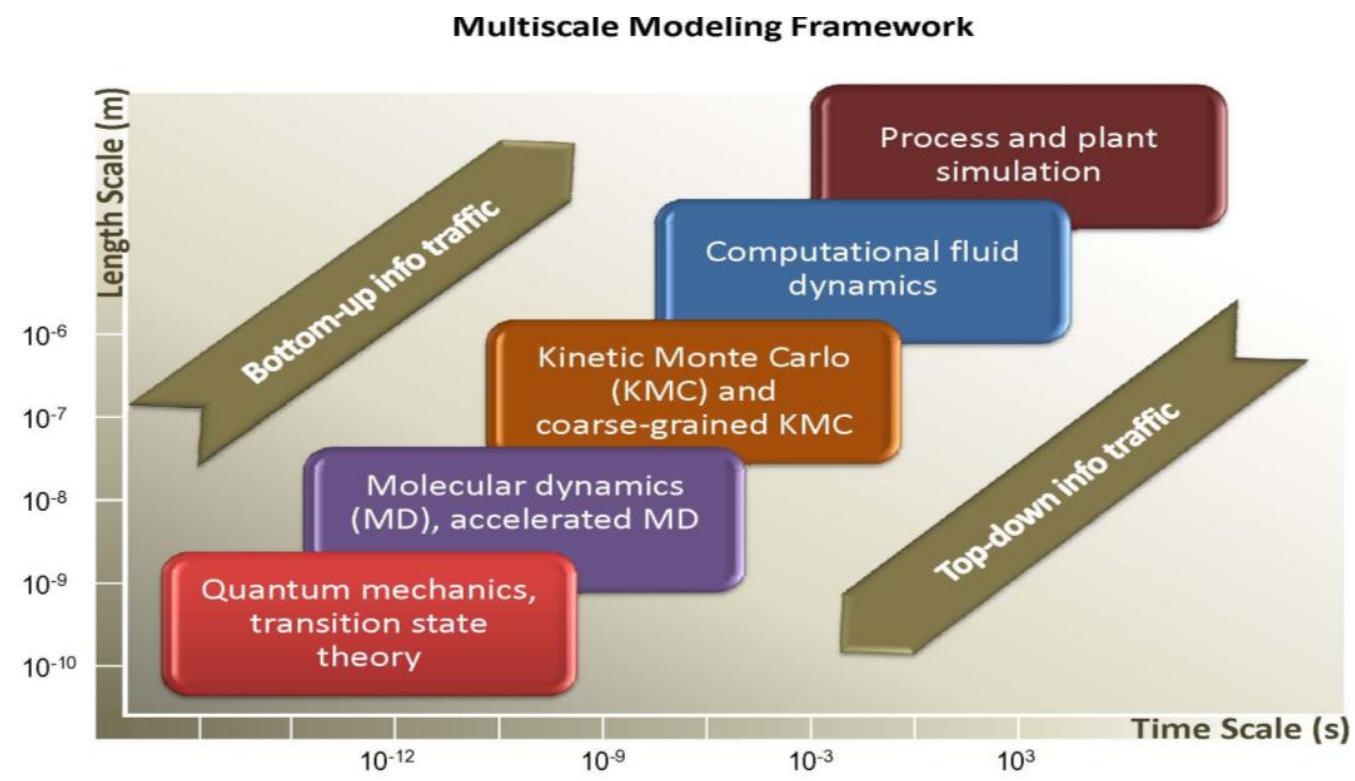

Figure 2. Schematic of various models operating at various scales. Redrawn from (Vlachos, 2005). 

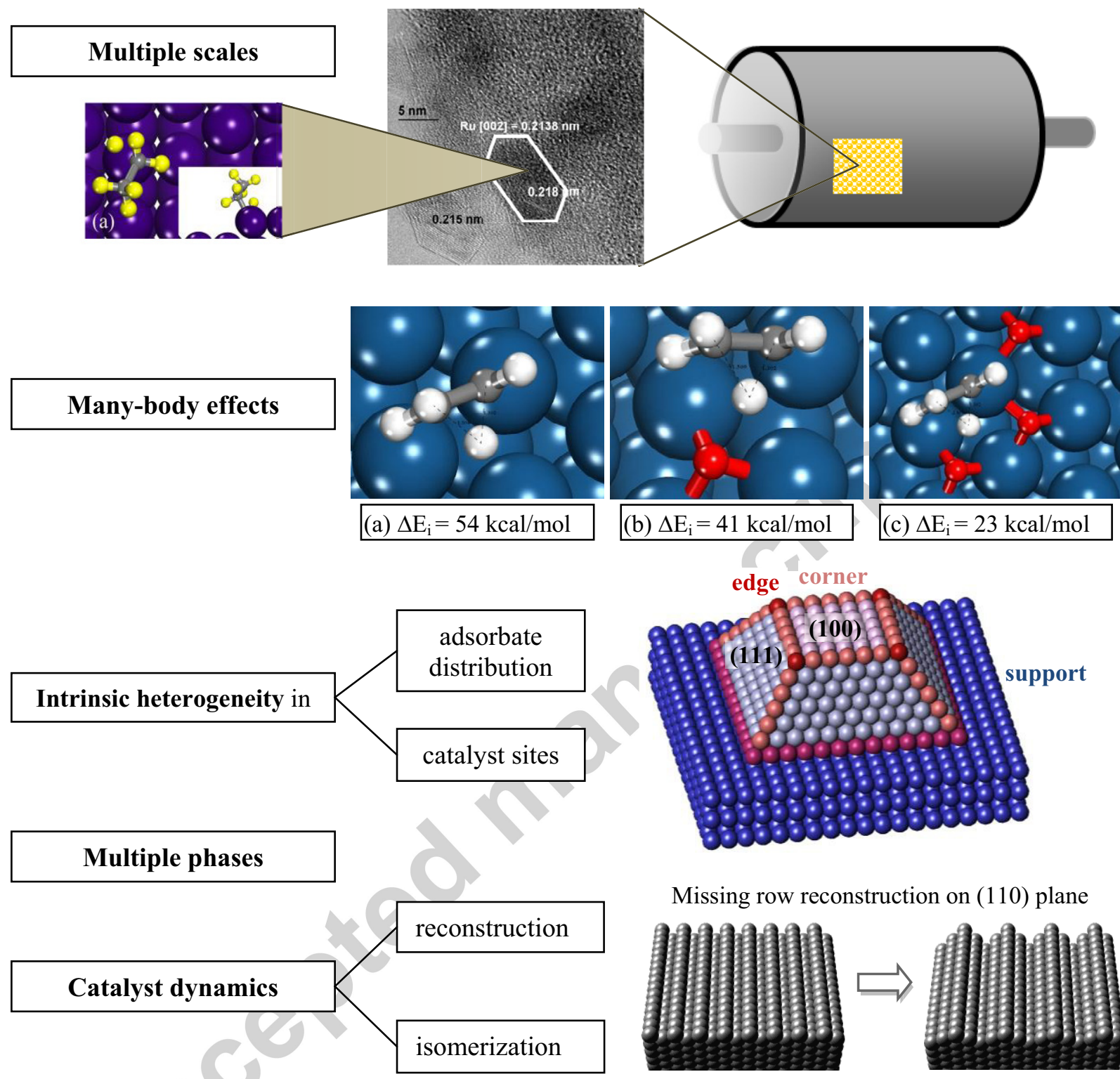

Missing row reconstruction on (110) plane
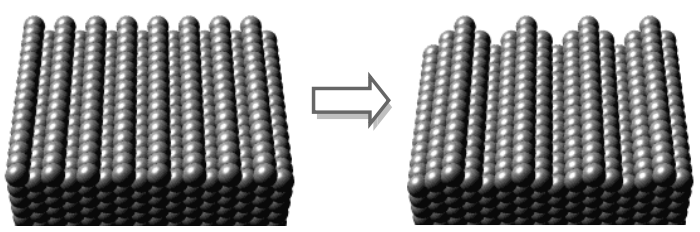

Figure 3. Overview of challenges in the multiscale modeling of catalytic systems and specific examples. In the many body effects, selected configurations (top view) in the presence of 0,1 , and 3 oxygen atoms for the $1,2 \mathrm{H}$ shift reaction (ethylene isomerization of $\mathrm{CH}_{2} \mathrm{CH}_{2} \rightarrow \mathrm{CHCH}_{3}$ ) and corresponding activation energy (kcal/mol) from periodic DFT calculations on $\mathrm{Pt}(111)$ indicate that the presence of co-adsorbates can strongly affect the reaction barriers. White: $\mathrm{H}$ atoms, gray: $\mathrm{C}$ atoms, red: $\mathrm{O}$ atoms. 


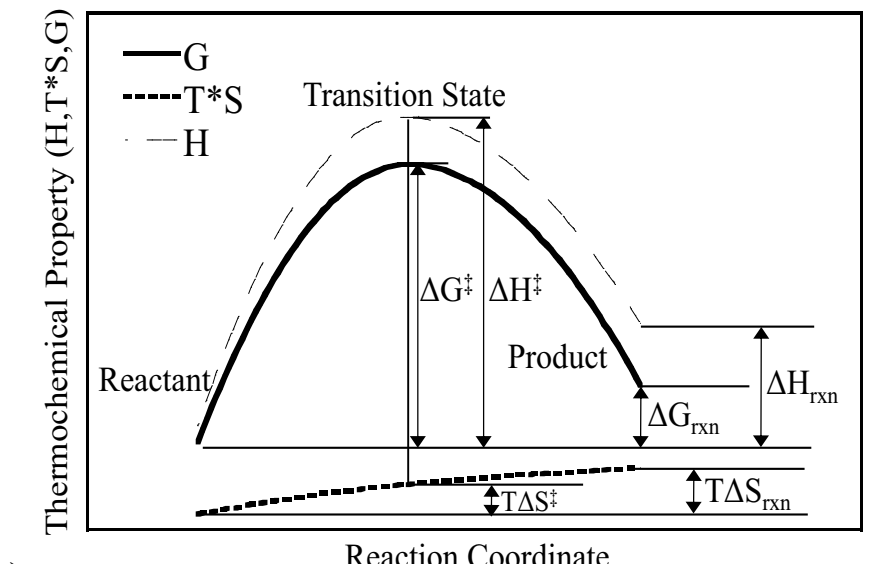

a)

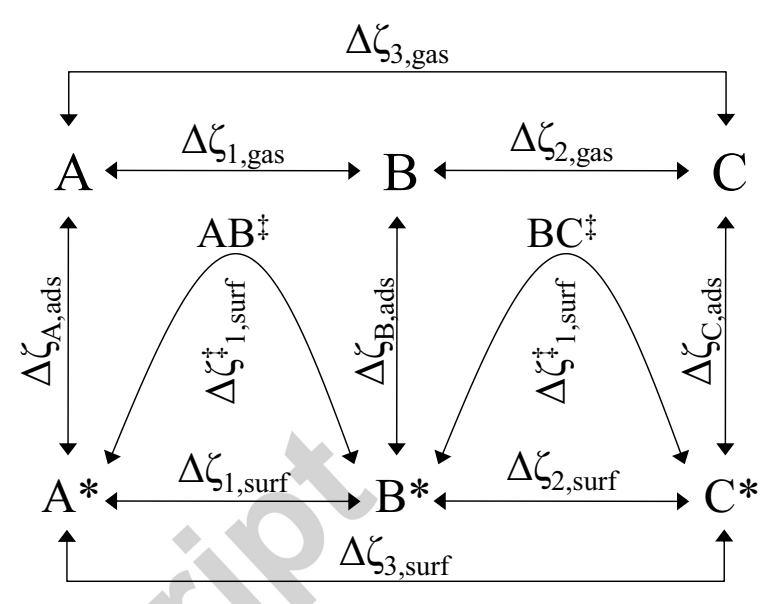

b)

Figure 4. Schematic of sample thermochemical property evolution with elementary reaction progression (a). Diagram of thermochemical property changes in the simple $A \leftrightarrow B \leftrightarrow C$ surface reaction mechanism (b). 


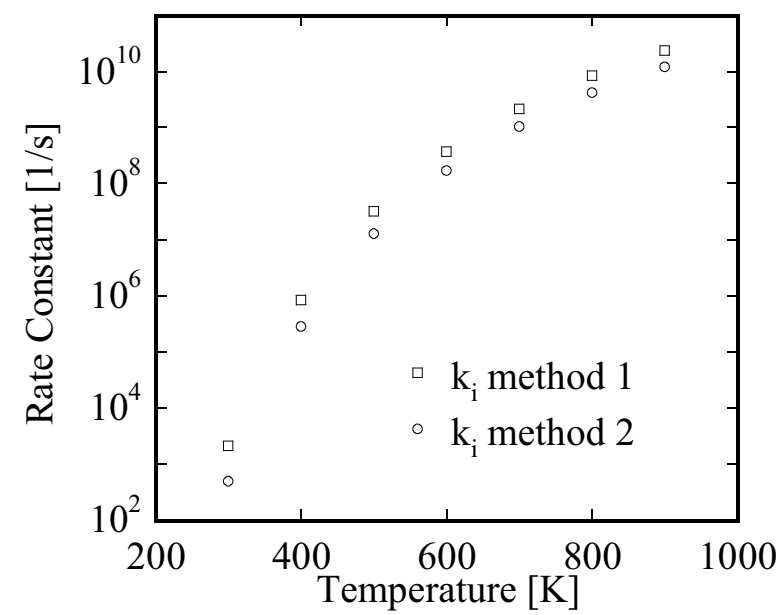

Figure 5. Comparison of thermodynamic consistency enforcement methods for $\mathrm{CH}_{2}{ }^{*}+\mathrm{H}^{*} \rightarrow \mathrm{CH}_{3}{ }^{*}$ reaction on Ni(111). Methods 1 and 2 refer to adjustment of surface reactions or adsorption properties, respectively, to match the surface thermochemistry to the gas-phase one (see text in Section 4.1). Information for assessment taken from (Blaylock et al., 2009). 


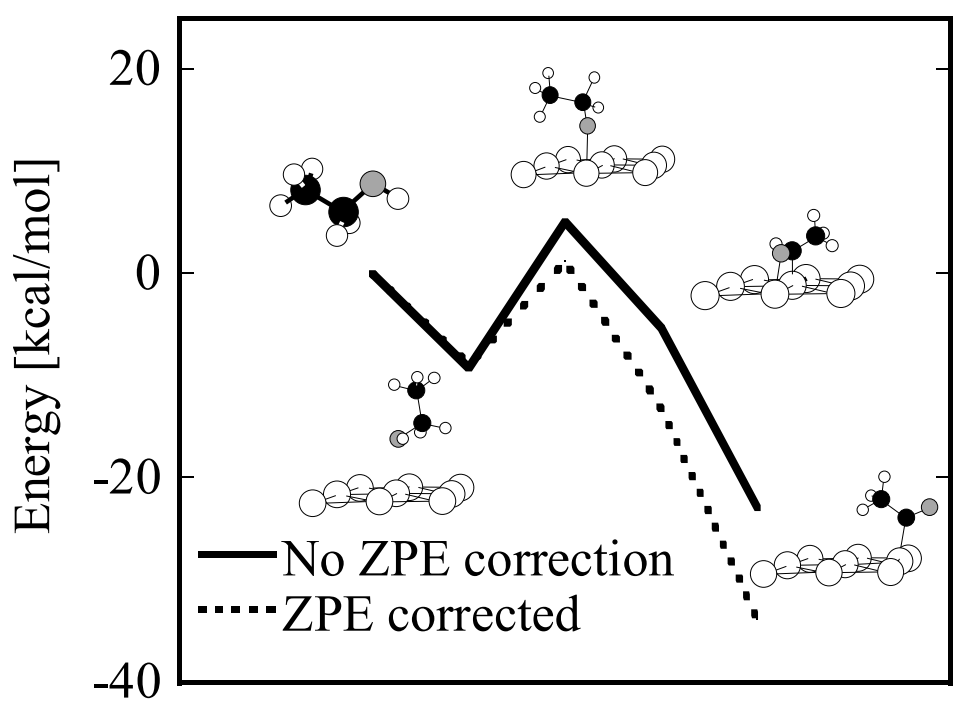

Figure 6. Comparison of energy profile for initial dehydrogenation reactions of ethanol on $\mathrm{Pt}(111)$ $\left(\mathrm{CH}_{3} \mathrm{CH}_{2} \mathrm{OH} \rightarrow \mathrm{CH}_{3} \mathrm{CH}_{2} \mathrm{O}+\mathrm{H} \rightarrow \mathrm{CH}_{3} \mathrm{CHO}+2 \mathrm{H} \rightarrow \mathrm{CH}_{3} \mathrm{CO}+3 \mathrm{H}\right)$ with and without $\mathrm{ZPE}$ corrections. All energies taken with respect to gas-phase ethanol and hydrogen adsorbed on a separate slab (hydrogen is excluded from graph for clarity). Energetic values and vibrational frequencies taken from (Salciccioli et al., 2011). 


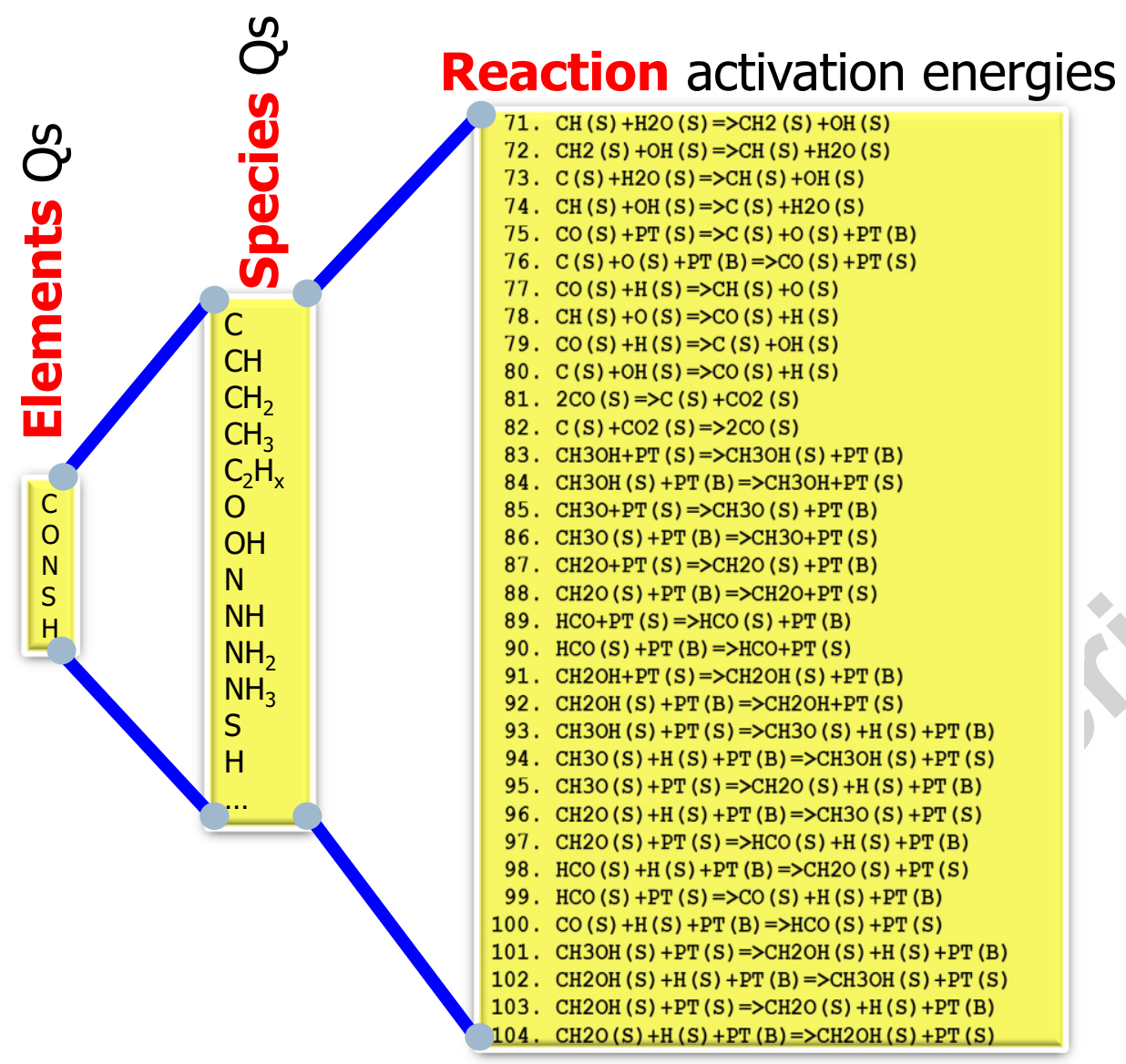

Figure 7. Schematic of mapping from atomic binding energies $Q$ to molecular species binding energies $Q$ (left map) and from molecular species binding energies to activation energies of a microkinetic model (right map). These mappings can be materialized either via the bond-order conservation method (used for both maps) or via linear scaling relations and group additivity (left map) and Brønsted-Evans-Polanyi (BEP) relations (right map). These relations can be derived via DFT. The double mapping compounded with metal transferability (Figure 9) enables optimization for optimal catalyst property identification (

Figure 21). 


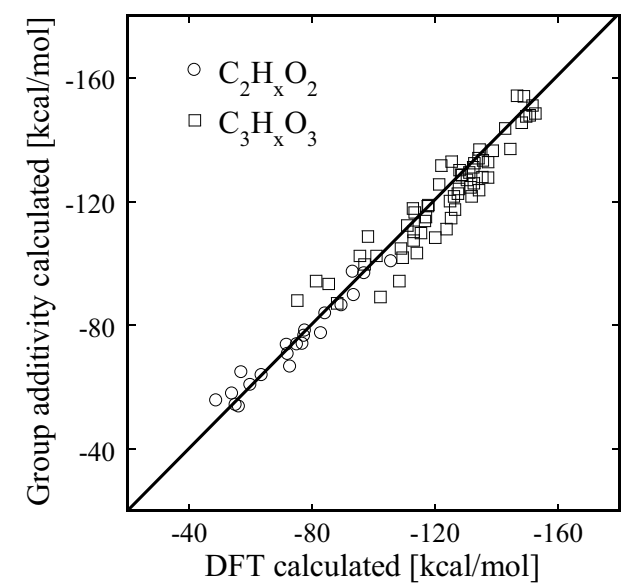

Figure 8. Comparison of group additivity calculated and DFT calculated $\mathrm{H}_{\mathrm{f}, 298}$ for $\mathrm{C} 2$ and $\mathrm{C} 3$ oxygenates. Replotted from (Salciccioli et al., 2010). 

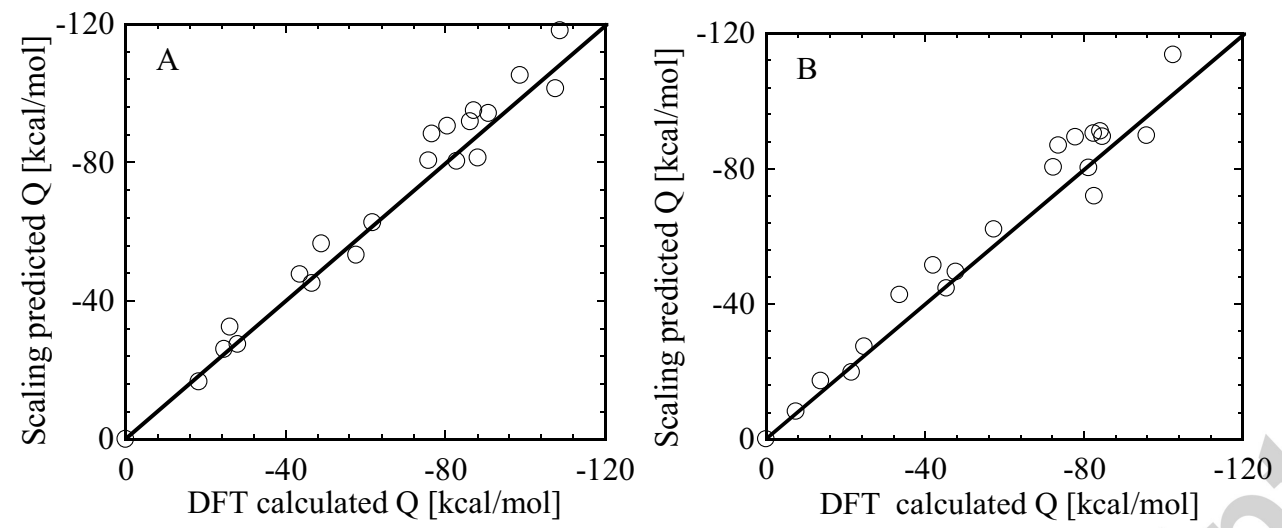

Figure 9. Transferability of thermochemistry from a single metal to other single and bimetallics: Comparison of energy of adsorption for $\mathrm{C}_{2} \mathrm{H}_{\mathrm{x}} \mathrm{O}_{2}$ species estimated from linear scaling relationships (Eq. 27) to DFT calculated energy of adsorption for (A) the Ni-Pt-Pt(111) bimetallic surface and for (B) the Ni(111) surface. Re-plotted from (Salciccioli et al., 2010). 


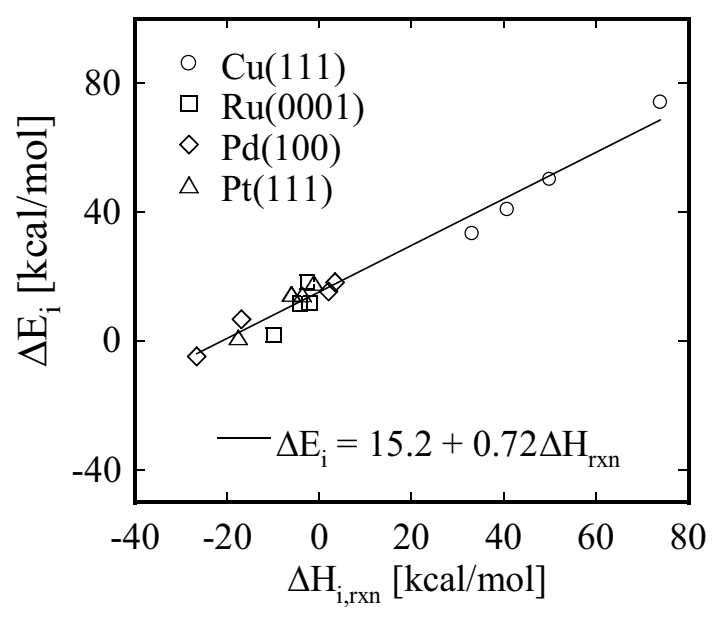

Figure 10. Demonstration of BEPs in terms of reaction barrier vs. heat of reaction. BEP for $\mathrm{CH}_{\mathrm{x}}$ dehydrogenation reactions (data from (Michaelides et al., 2003) and line is obtained by regression). 


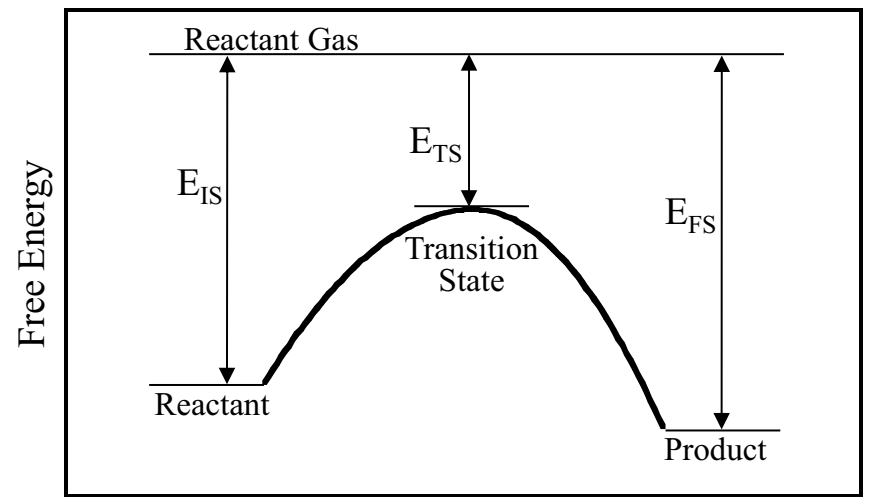

Reaction Coordinate

Figure 11. Elementary reaction diagram showing the definition of initial state $\left(\mathrm{E}_{\mathrm{IS}}\right)$, transition state $\left(\mathrm{E}_{\mathrm{TS}}\right)$ and final state $\left(\mathrm{E}_{\mathrm{FS}}\right)$ energies for linear free energy relationship (Alcalá et al., 2003). 


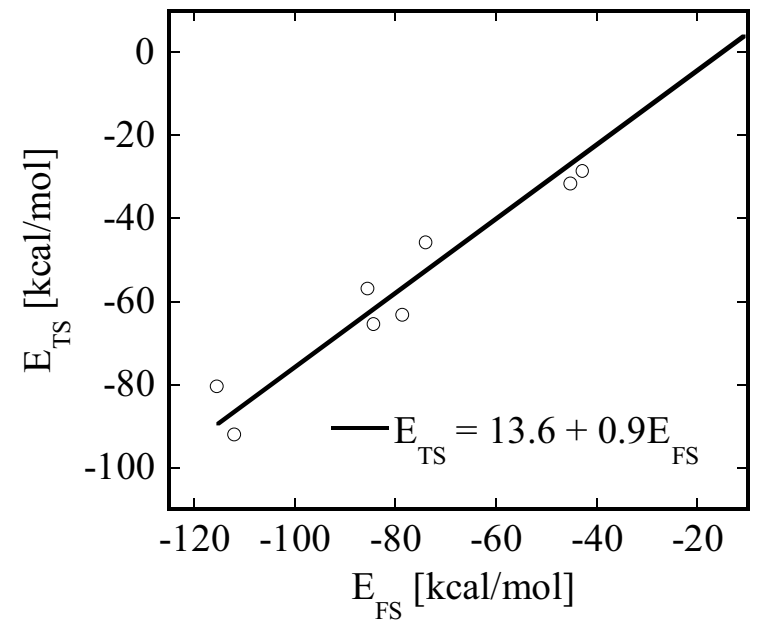

Figure 12. Demonstration of BEPs in terms of transition state energy vs. final state energy: Transition state energies as a linear function of final state energies (both referenced from the gas-phase initial state) for methanol dehydrogenation reactions on Pt. Data replotted from (Greeley and Mavrikakis, 2004b). 

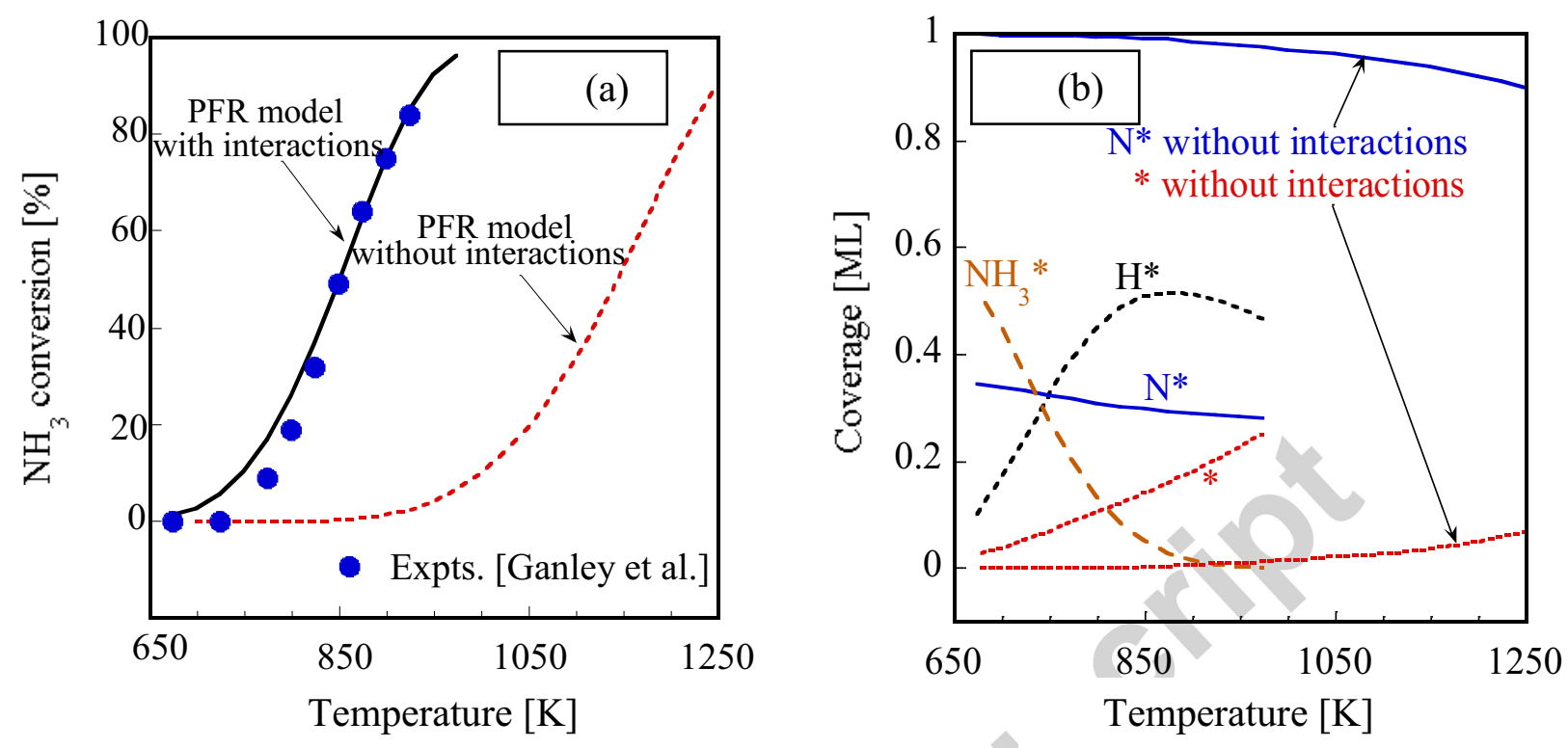

Figure 13. Effect of interactions in ammonia decomposition chemistry. (a): Conversion vs. temperature without interactions (red, dashed line; first modeling iteration) and with interactions (solid, black line; refined model in second iteration) along with experimental data (blue circles). (b): Most abundant species without interactions (adsorbed $\mathrm{N}$ dominates) and with interactions (adsorbed $\mathrm{H}$ and $\mathrm{N}$ dominate at moderate temperatures). Taken from (Mhadeshwar et al., 2004). 

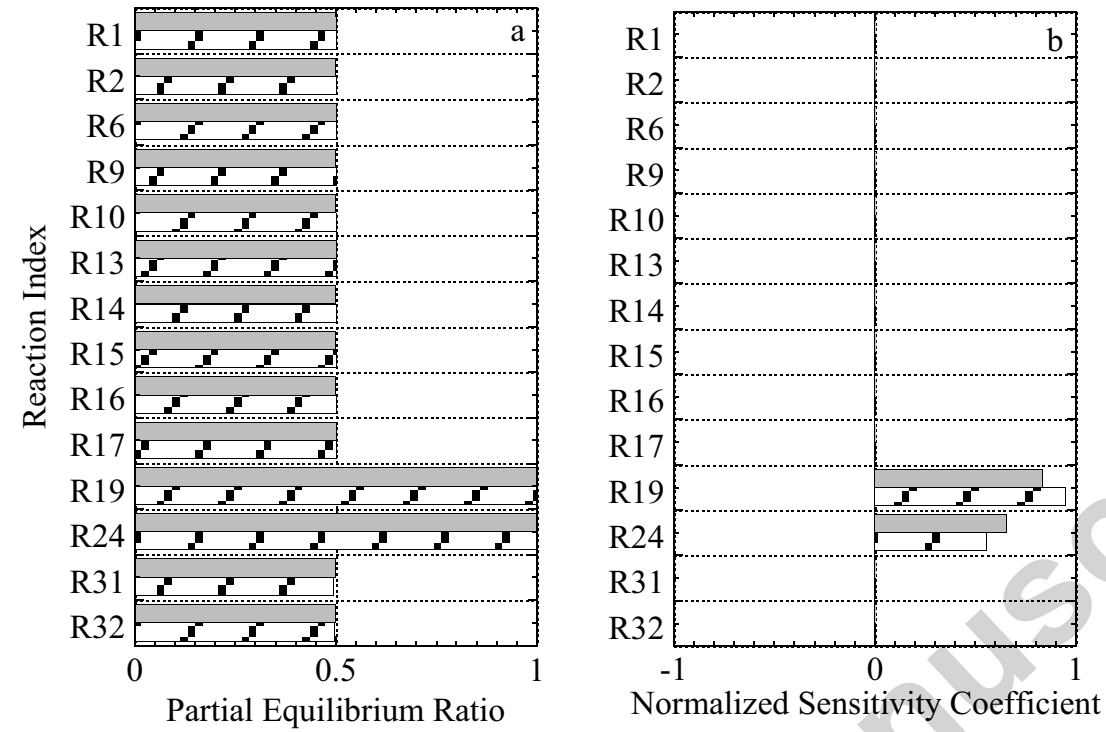

Figure 14. a) Partial equilibrium ratio (Eq. 31) and b) normalized sensitivity coefficients (Eq. 32) at $623 \mathrm{~K}$ for ethane hydrogenolysis. Analysis done at two feed conditions: $\mathrm{P}_{\mathrm{H} 2}=25$ Torr and $\mathrm{P}_{\mathrm{C} 2 \mathrm{H} 6}=25$ Torr (shaded) and $\mathrm{P}_{\mathrm{H} 2}=349$ Torr and $\mathrm{P}_{\mathrm{C} 2 \mathrm{H} 6}=122$ Torr (striped). Data replotted from (Salciccioli et al., 2011). 

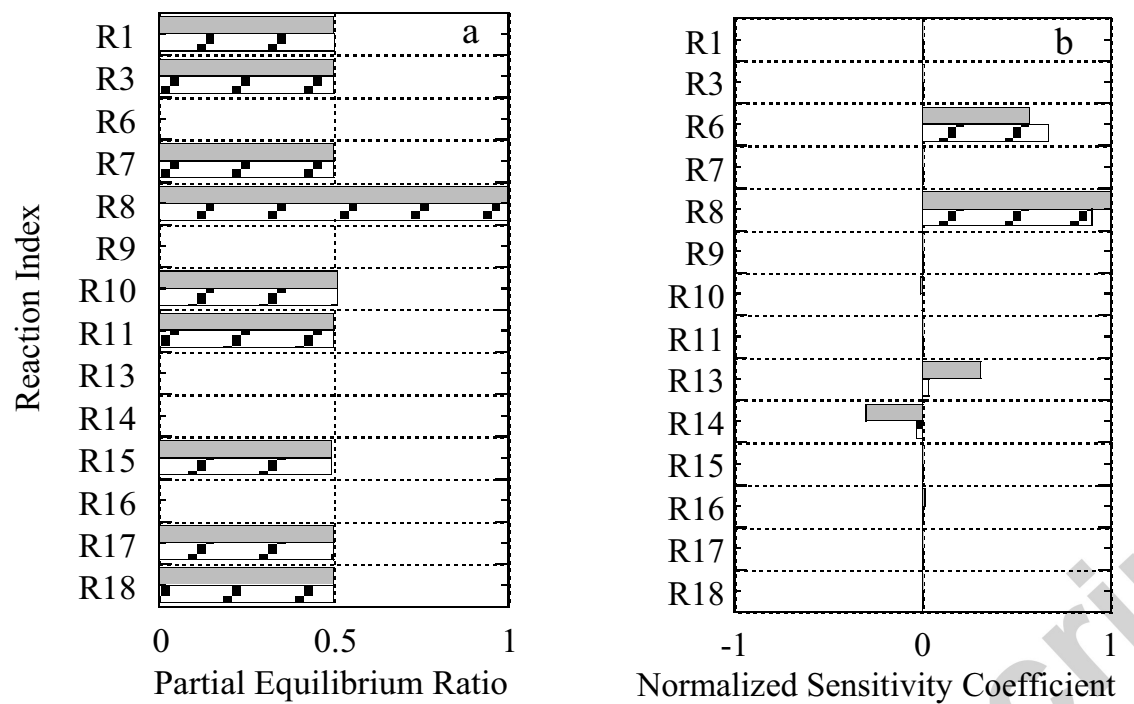

Figure 15. a) Partial equilibrium ratio (Eq. 31) of each reaction at $298 \mathrm{~K}$ and b) normalized sensitivity coefficient (Eq. 32) of each reaction at $298 \mathrm{~K}$ for ethylene hydrogenation. Analysis done at two conditions of varying hydrogen pressure: $\mathrm{P}_{\mathrm{H} 2}=50$ Torr (shaded) and $\mathrm{P}_{\mathrm{H} 2}=665$ Torr (striped). Data replotted from (Salciccioli et al., 2011). 


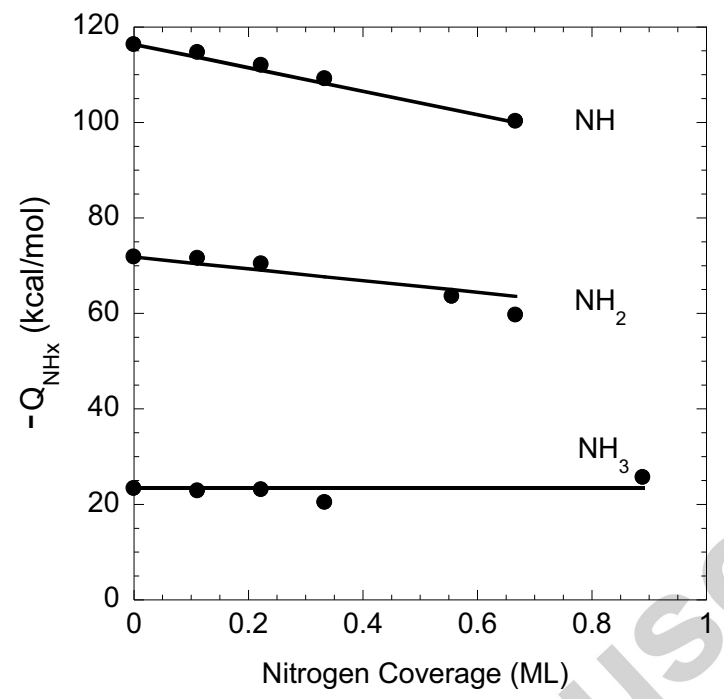

Figure 16. DFT binding energies (points) of $\mathrm{NH}_{\mathrm{x}}$ species as a function of increasing atomic $\mathrm{N}$ coverage compared to binding energies predicted through scaling relationships (lines). Taken from (Hansgen, 2011). 


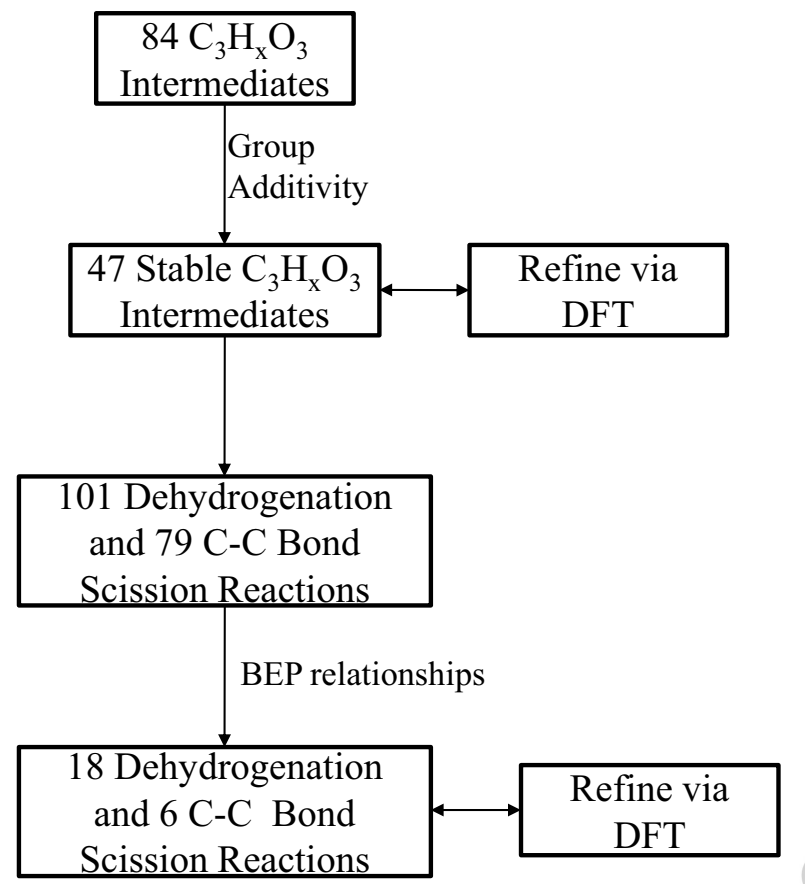

Figure 17. Schematic of hierarchical refinement methodology showing progression from a broad data set to an accurately computed glycerol decomposition reaction path on Pt. Redrawn from (Chen et al., Submitted). 


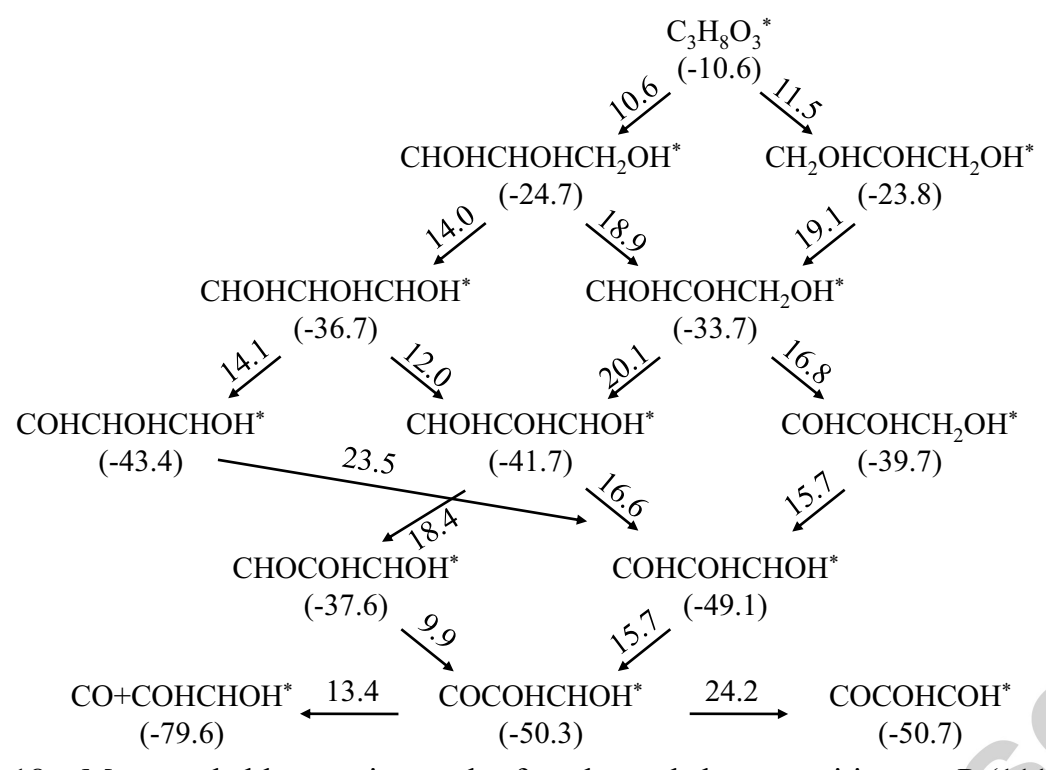

Figure 18. Most probable reaction paths for glycerol decomposition on $\operatorname{Pt}(111)$ calculated via DFT. Activation barriers for elementary reactions are shown above arrows $(\mathrm{kcal} / \mathrm{mol})$. The electronic energy of each intermediate is shown in parenthesis $(\mathrm{kcal} / \mathrm{mol})$ relative to glycerol in vacuum and hydrogen adsorbed on separate slabs. Excess hydrogen atoms are not shown in reactions for clarity. Redrawn from (Chen et al., Submitted). 


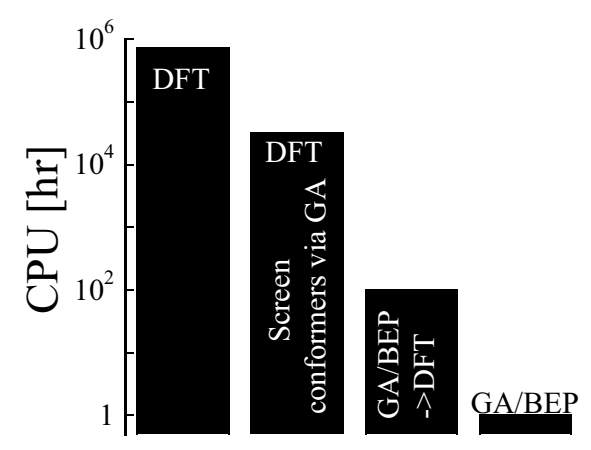

Figure 19. Estimated computational costs of glycerol decomposition mechanism development. The 'GA/BEP' value represents use of semi-empirical methods for mechanism development (this value is set arbitrarily to 1 , as it carries very little cost). The first hybrid value ('screen conformers via GA') group additivity is used to screen for the most stable conformers, followed by DFT refinement for important species and all of their reactions. In the 'GA/BEP $\rightarrow$ DFT' method, important species and important reactions are identified followed by DFT refinement. The "DFT" column estimates the expense of computing all mechanism parameters via DFT. A CPU hour unit is the estimated expense associated with utilizing one processor for one hour. Redrawn from (Chen et al., Submitted). 


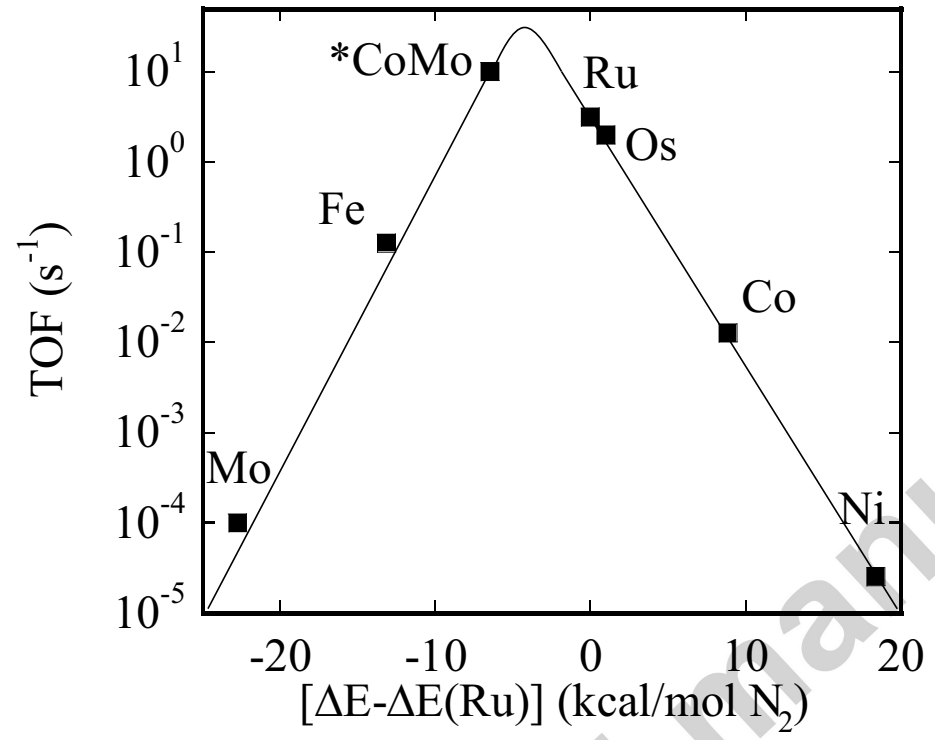

Figure 20. Volcano curve for prediction of optimal catalyst for $\mathrm{NH}_{3}$ synthesis. The line is a guide to the eye of model-predicted data. The synthesis conditions are $400{ }^{\circ} \mathrm{C}$, 50 bar, gas composition $\mathrm{H}_{2}: \mathrm{N}_{2}=3: 1$ containing $5 \% \mathrm{NH}_{3}$. Taken from (Jacobsen et al., 2001). 

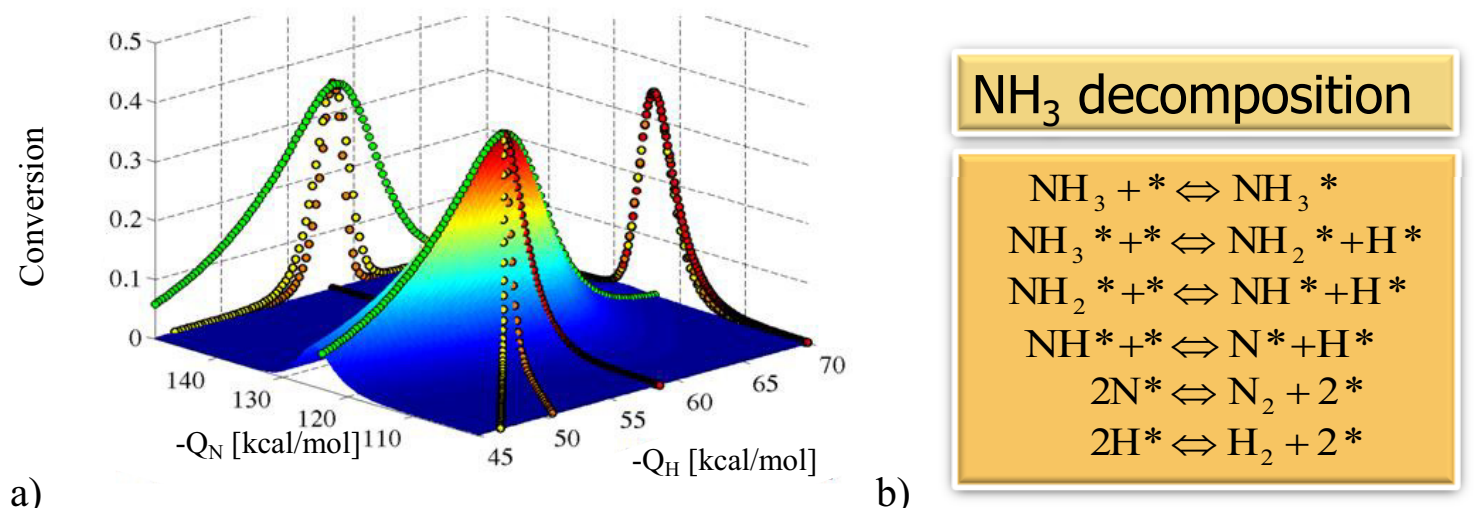

a)

b)

Figure 21: (a) Volcano mountain: conversion of $\mathrm{NH}_{3}$ vs. the atomic heats of adsorption computed via Monte Carlo optimization (searching randomly in binding energies and solving the microkinetic model at each pair of binding energies) using a microkinetic model in a plug-flow reactor model. (b) Elementary reaction mechanism. Taken from (Ulissi et al., 2010). 

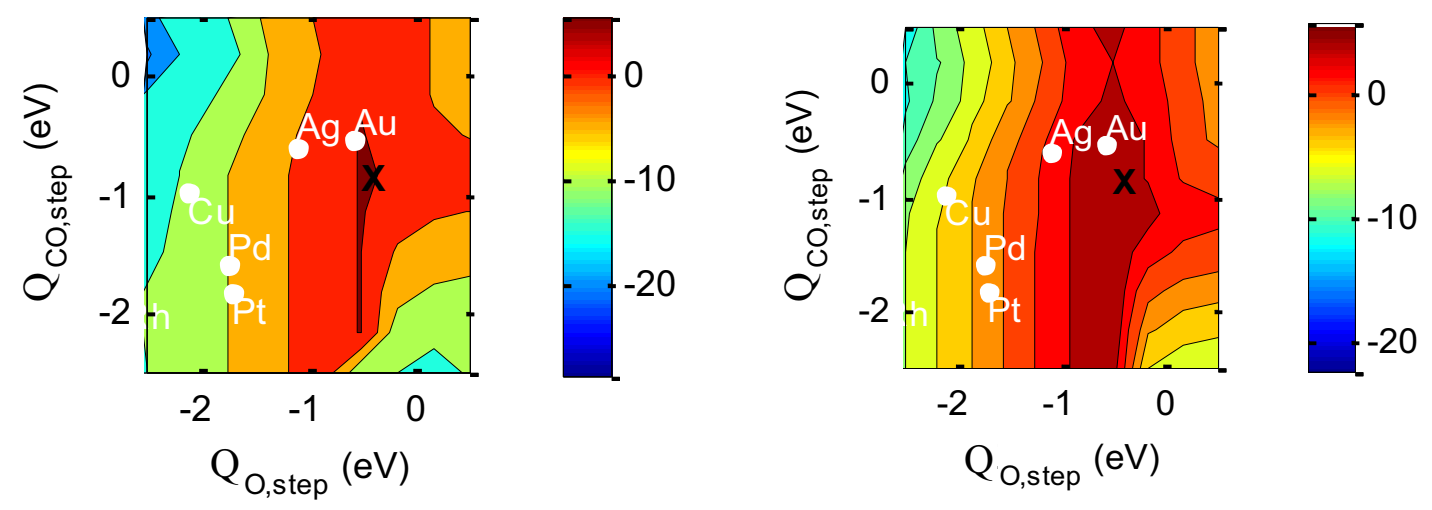

Figure 22. Log of TOF for $\mathrm{CO}_{2}$ formation on steps without lateral interaction at $600 \mathrm{~K}$ (left) and $800 \mathrm{~K}$ (right). Reaction conditions: $\mathrm{P}_{\mathrm{O} 2}=0.3$ bar, $\mathrm{P}_{\mathrm{CO} 2}=0.04$ bar, $\mathrm{P}_{\mathrm{CO}} / \mathrm{P}_{\mathrm{O} 2}=2, \mathrm{~T}=600 \mathrm{~K}$. Optimal properties are $\mathrm{Q}_{\mathrm{O}}=-0.6 \mathrm{eV}, \mathrm{Q}_{\mathrm{CO}}=-$ $0.8 \mathrm{eV}$ (left) and $\mathrm{Q}_{\mathrm{O}}=-0.5 \mathrm{eV}, \mathrm{Q}_{\mathrm{CO}}=-1.2 \mathrm{eV}$ (right). 

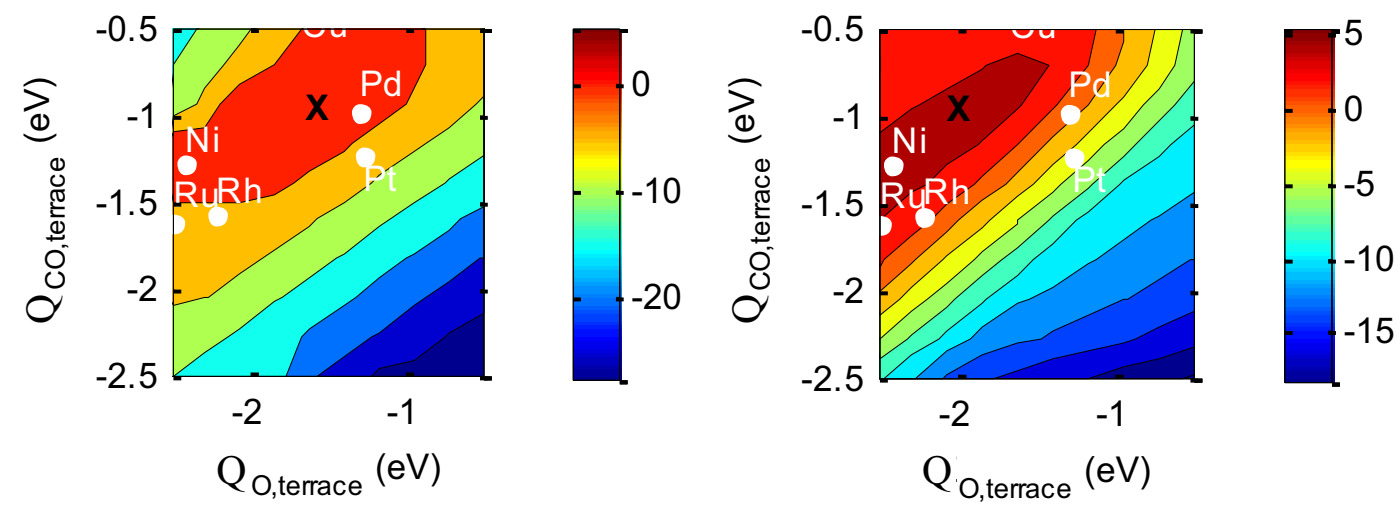

Figure 23. $\mathrm{Log}$ of TOF for $\mathrm{CO}_{2}$ formation on terraces without lateral interactions (left) and with lateral interactions (right). Reaction conditions: $\mathrm{P}_{\mathrm{O} 2}=0.3$ bar, $\mathrm{P}_{\mathrm{CO} 2}=0.04$ bar, $\mathrm{P}_{\mathrm{CO}} / \mathrm{P}_{\mathrm{O} 2}=2, \mathrm{~T}=600 \mathrm{~K}$. The optimal properties are $\mathrm{Q}_{\mathrm{O}}=-1.5$ $\mathrm{eV}$ and $\mathrm{Q}_{\mathrm{CO}}=-0.9 \mathrm{eV}$ in the absence of interactions and $\mathrm{Q}_{\mathrm{O}}=-2 \mathrm{eV}, \mathrm{Q}_{\mathrm{CO}}=-0.95 \mathrm{eV}$ when interactions are accounted for. 


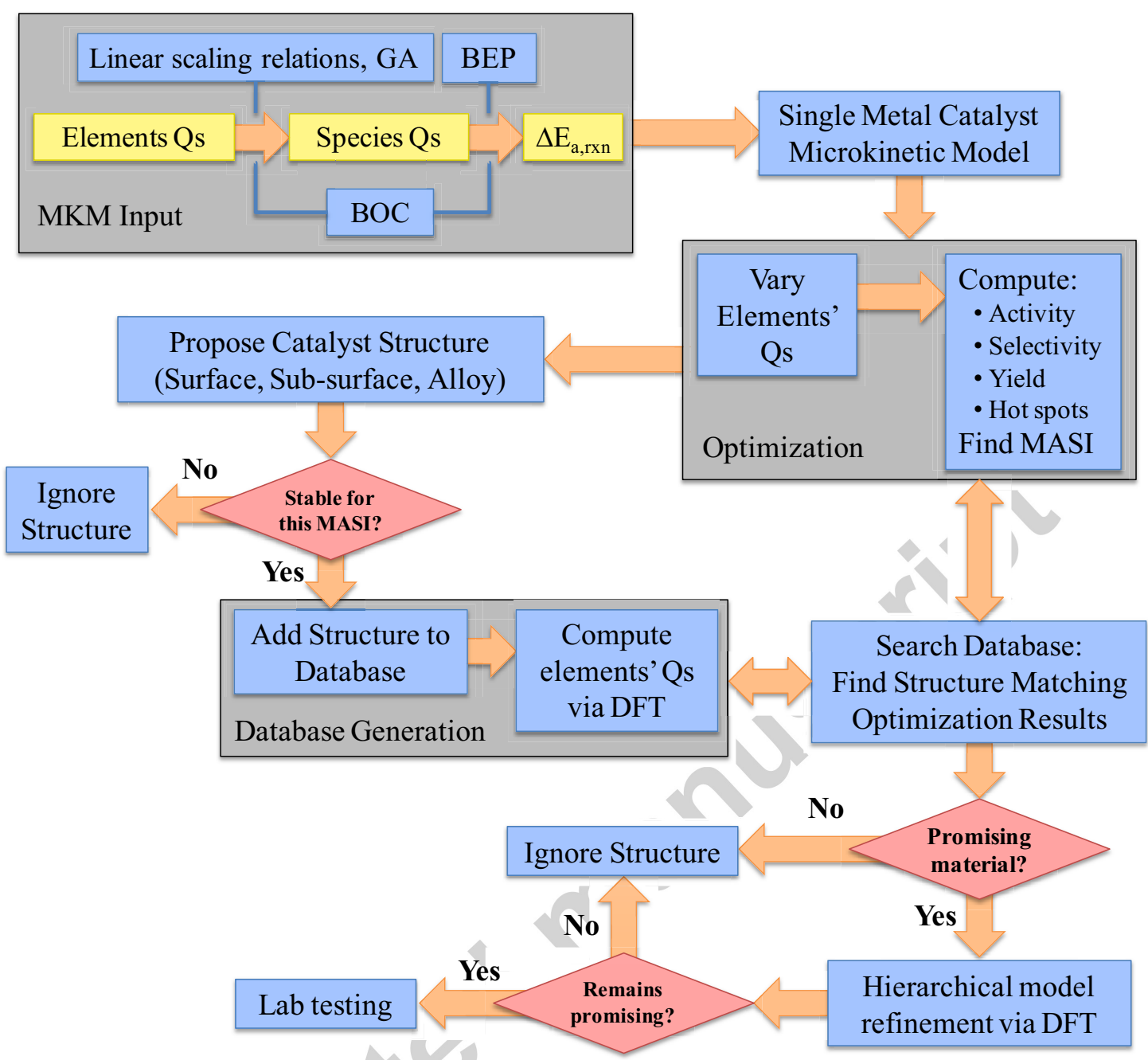

Figure 24. Proposed computational approach for the identification of novel catalyst formulations and surface structures. The approach combines microkinetic modeling (MKM), input via semi-empirical methods, such as the bond-order conservation (BOC), group additivity (GA), linear scaling relations and Brønsted-Evans-Polanyi (BEP) relations derived from DFT, an optimization with respect to atomic binding energies, stability studies via DFT and/or MD under working conditions (based on most abundant surface intermediate), database generation of binding energies via DFT on different structures, materials selection based on optimization and database results, hierarchical refinement of the models of novel materials via higher level theory, and lab testing for promising candidates. 


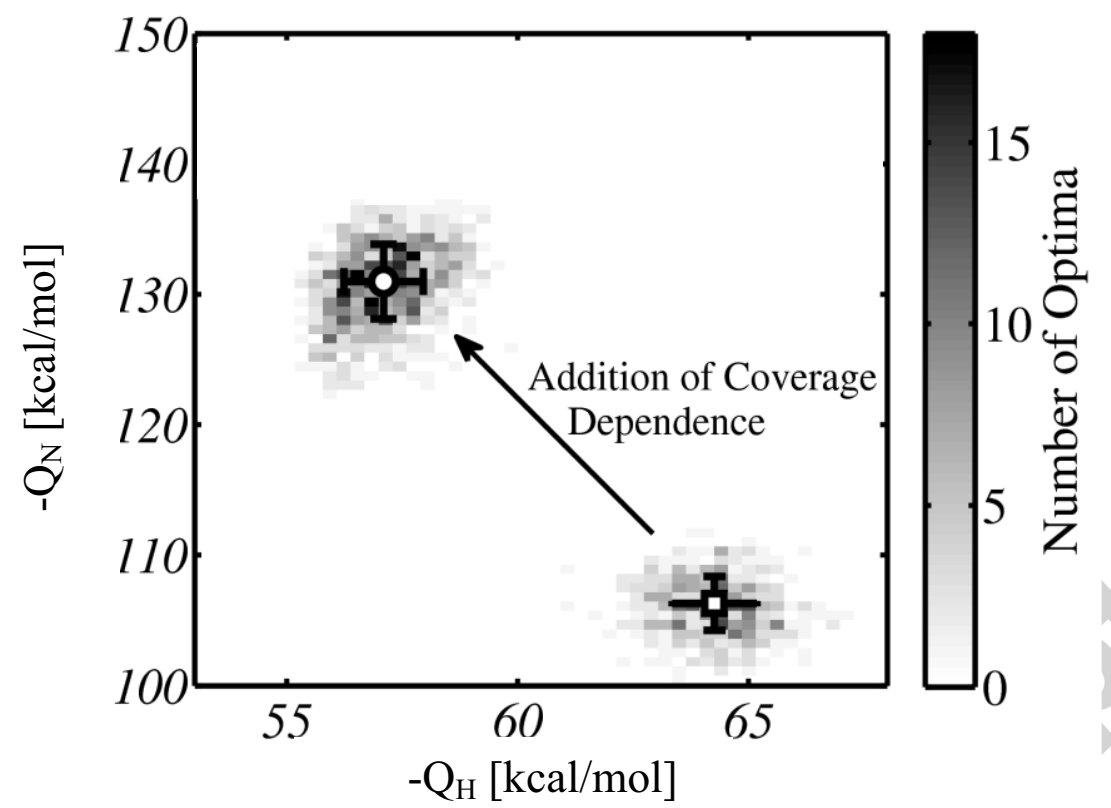

Figure 25. Uncertainty map for the $\mathrm{NH}_{3}$ decomposition reaction. The distribution indicates the location of optimal binding energies of $\mathrm{N}$ and $\mathrm{H}$ (i.e., the probability distribution) due to uncertainty in pre-exponentials. The crosses indicate the standard deviation of the distribution. Uncertainty in most parameters of the microkinetic model has a slight effect of the optimal properties. The inclusion of lateral interactions shifts significantly the optimal catalyst properties of $-\mathrm{Q}_{\mathrm{H}},-\mathrm{Q}_{\mathrm{N}}$ from $64.3 \pm 0.9,106.3 \pm 2.1$ to $57.1 \pm 0.9,131 \pm 2.9 \mathrm{kcal} / \mathrm{mol}$. Taken from (Ulissi et al., 2010). 


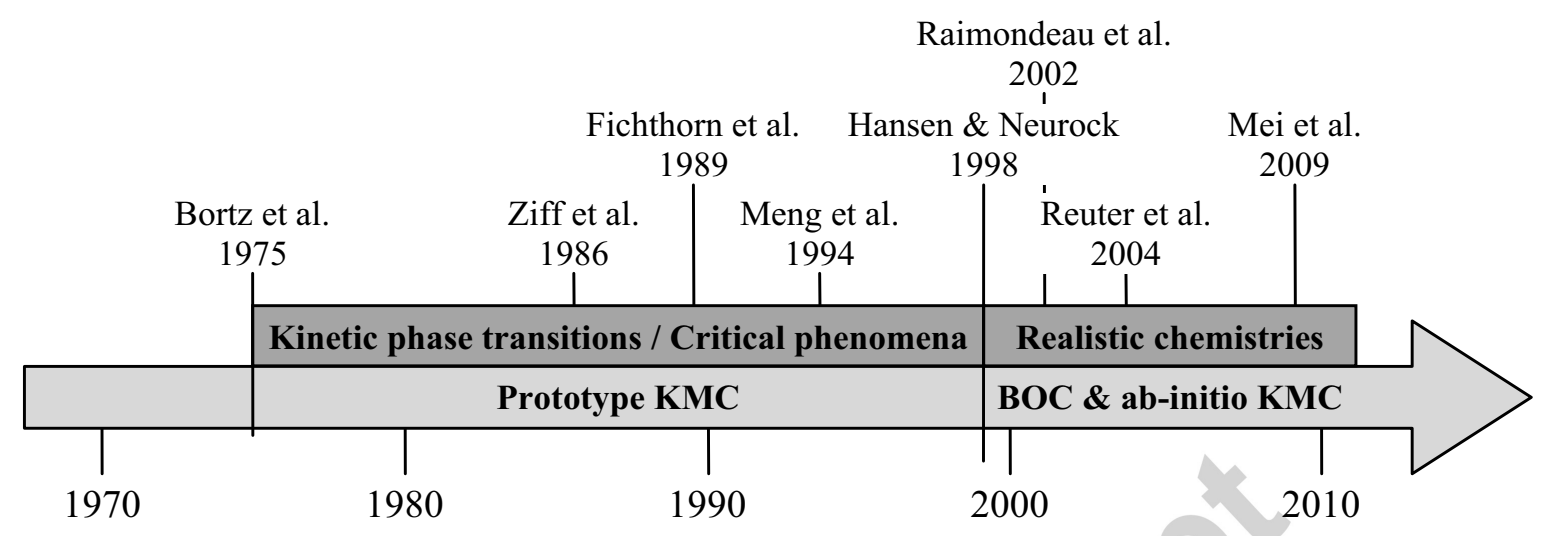

Figure 26. Timeline for the development of kinetic Monte Carlo (KMC) methods. The KMC method, attributed to Bortz et al., was originally used for exploring kinetic phase transitions and critical phenomena. In these prototype KMC simulations, parameters were usually chosen arbitrarily. With the advent of BOC-KMC and $a b$ initio KMC methods in the late 90 's, realistic parameters were inputted making the investigation of complex chemistries possible. 
(a)

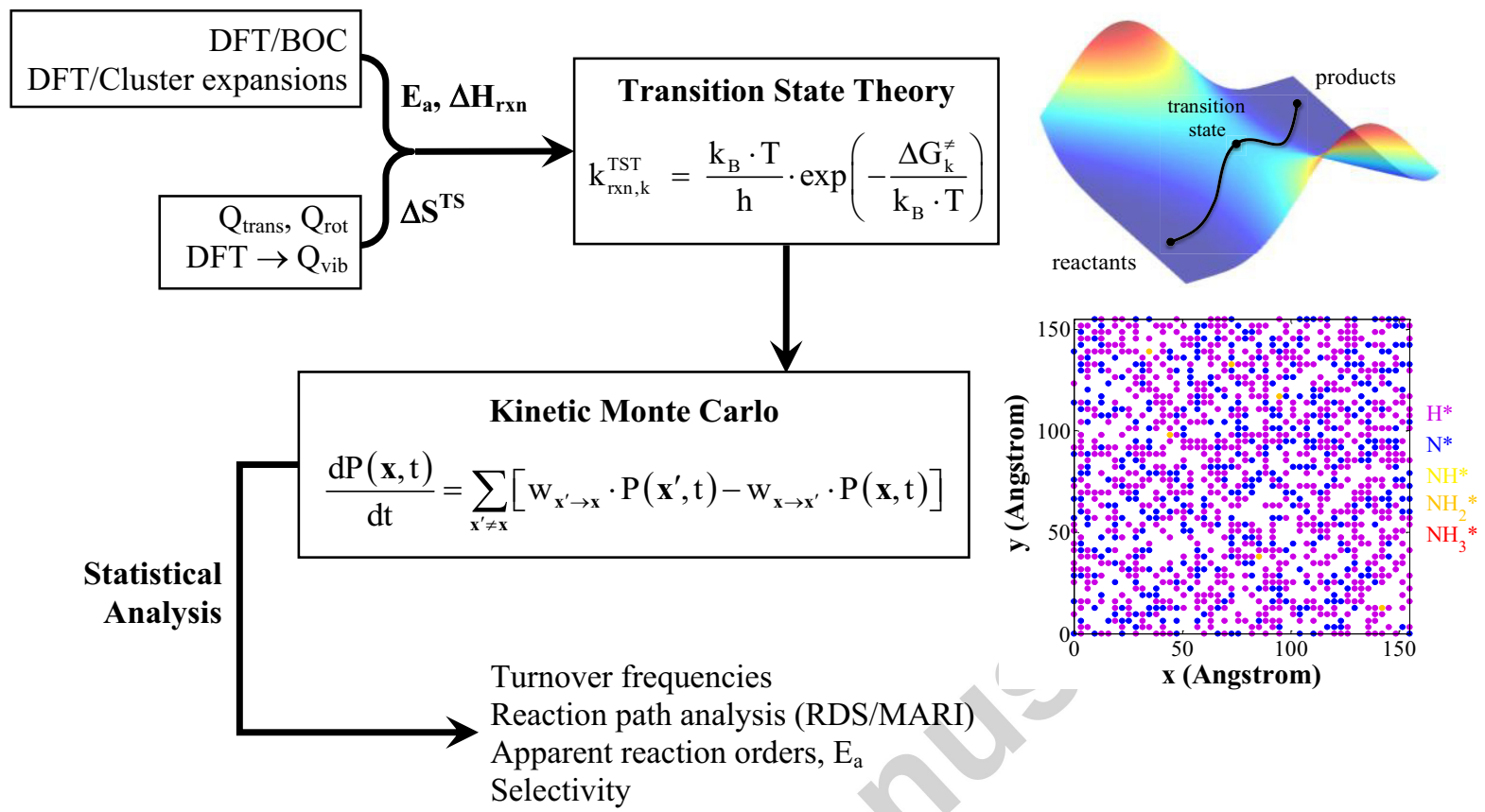

(b)

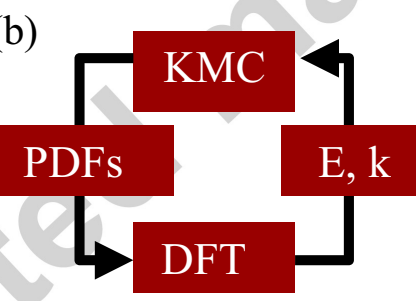

Figure 27. (a) Flow of information in a multiscale kinetic Monte Carlo (KMC) framework: density functional theory (DFT) provides the vibrational frequencies of adsorbates and transition states. The vibrational partition function $\mathrm{Q}_{\text {vib }}$ can thus be approximated, which along with the translational and vibrational components give an estimate of the entropy of the transition state. DFT also provides reaction and transition state energies, which can be used along with BOC and cluster expansions to calculate coverage-dependent activation energies for each elementary step. By using standard approximations within the framework of harmonic transition state theory, the kinetic rate constant of each elementary step is calculated. These constants are input to the kinetic Monte Carlo (KMC) framework that simulates stochastic paths of a discrete master equation. Statistical analysis of the KMC trajectories conveys information about the chemistry studied. This schematic ignores many-body effects. (b) Iterative scheme indicating how the KMC can compute the probability distribution function (pdf) of possible configurations on which the DFT can be carried out. Upon improving the input of KMC, the procedure is iterated until the configurations generated via the KMC have been parameterized via DFT. 


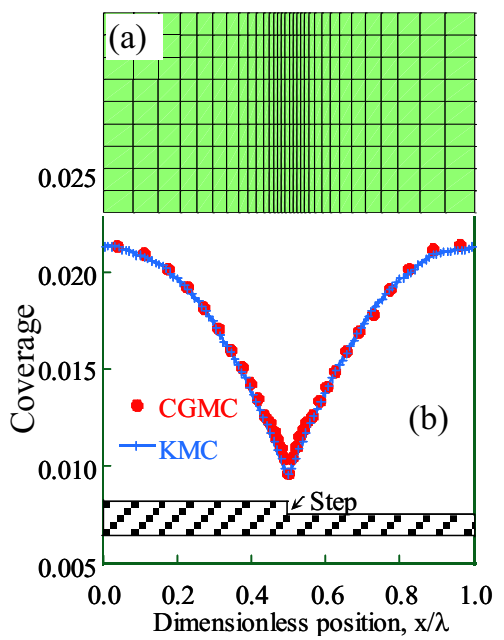

Figure 28. (a) Two-dimensional adaptive mesh used in coarse-grained kinetic Monte Carlo (CG-KMC) simulation to resolve high reactivity near a step. (b) Comparison of adaptive CG-KMC and microscopic KMC steady state solution averaged along the step. 

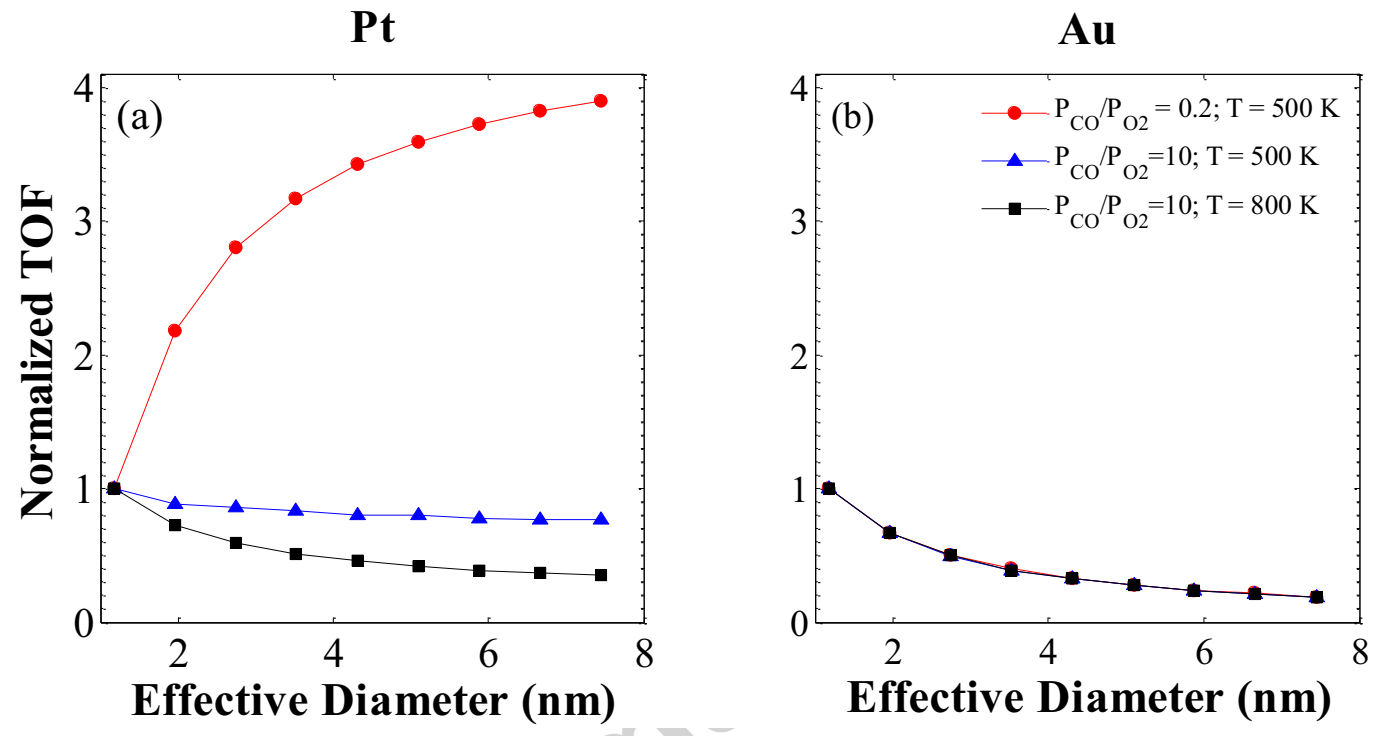

Figure 29. Normalized TOF of CO oxidation on $\mathrm{Pt}$ (a) and $\mathrm{Au}$ (b) vs. effective particle diameter, d, for various conditions noted in the legend (the latter pertains to both panels) using the mean-field microkinetic model. In all cases, $\mathrm{P}_{\mathrm{O} 2}=0.03$ bar and $\mathrm{P}_{\mathrm{CO} 2}=0.004$ bar. Due to the maximum fraction of steps on the smallest nanoparticle, the theoretical minimum and maximum values are around 0.2 and 5 of the normalized TOF. 


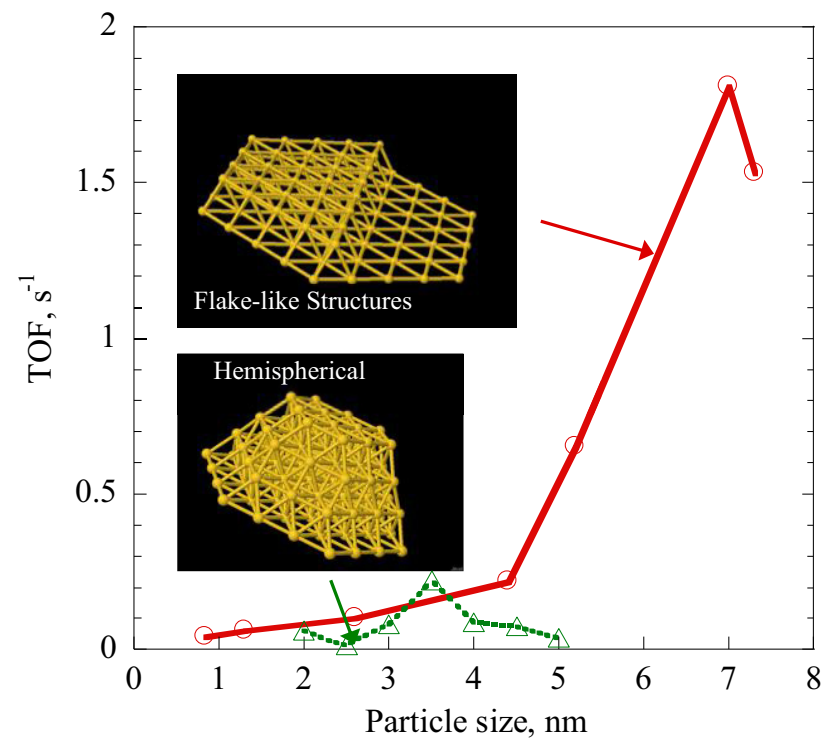

Figure 30. Size and shape sensitivity of the ammonia decomposition reaction on $\mathrm{Ru} / \gamma-\mathrm{Al}_{2} \mathrm{O}_{3}$ catalyst. Lines connect the simulation points. Redrawn from (Karim et al, 2009). 

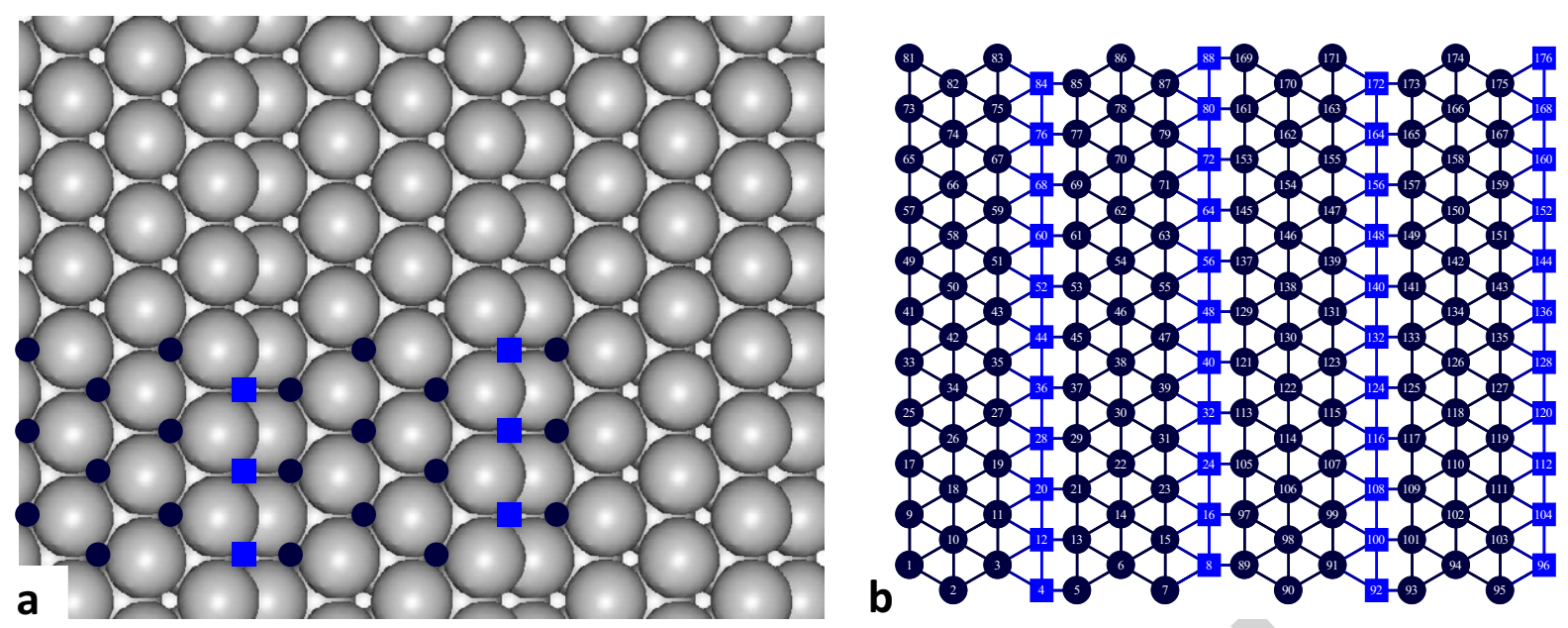

Figure 31. Au(533) surface (a) and the corresponding KMC lattice (b). For simplicity, only fcc hollow sites are considered here. Terrace sites are denoted with dark blue circles and step sites with blue squares. 

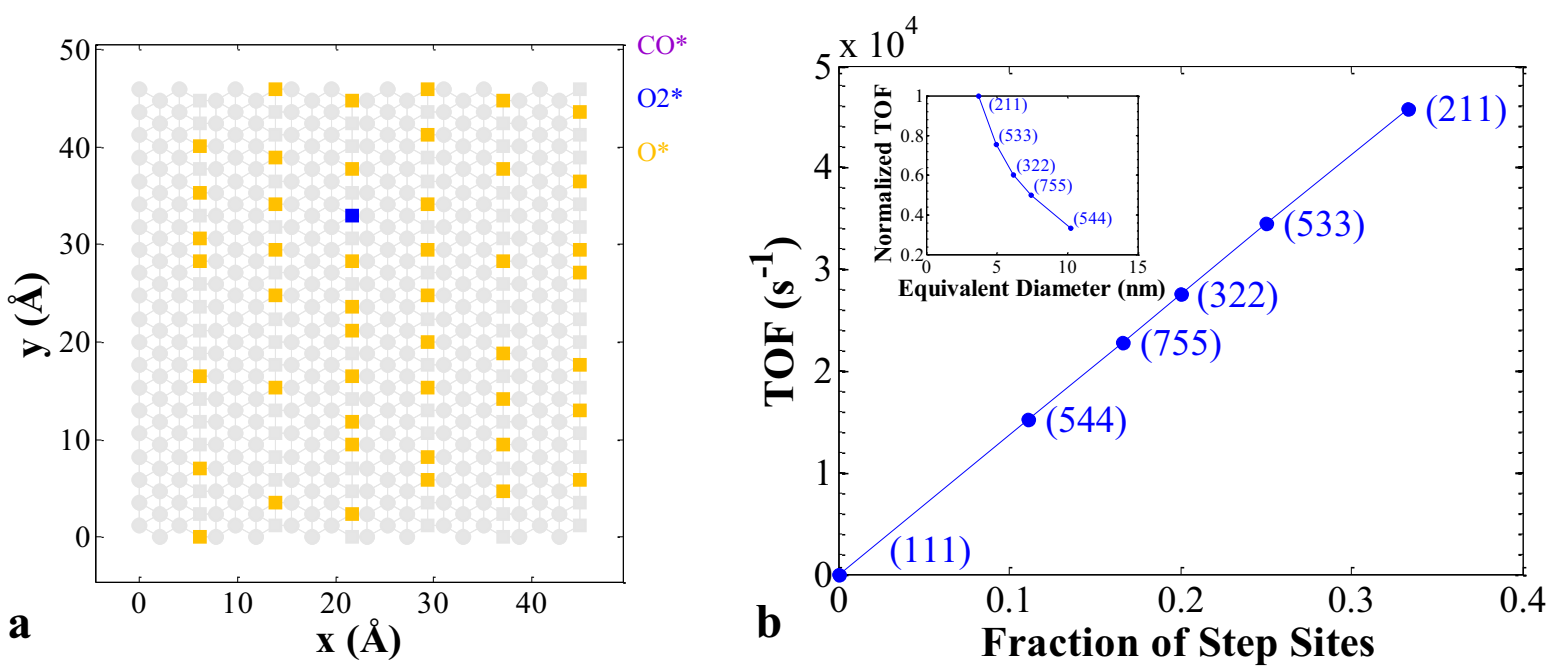

Figure 32. (a) Snapshot of the KMC simulation at steady state. The colored sites indicate occupied sites with adsorbates shown in the legend. (b) Turnover frequencies per monolayer with respect to step site density. The surface corresponding to each point in the plot is mentioned in parentheses next to the points. By mapping the step site density to an equivalent ratio for octahedral Au nanoparticles, the TOF can be plotted with respect to an equivalent particle diameter (see inset of panel b). Note that the (111) does not appear because it maps to an equivalent diameter of infinity. All simulations were performed at $300 \mathrm{~K}$ temperature and partial pressures $\mathrm{p}_{\mathrm{CO}}=$ $0.006, \mathrm{p}_{\mathrm{O} 2}=0.030, \mathrm{p}_{\mathrm{CO} 2}=0.004$ bar. A total of $0.025 \mathrm{~s}$ were simulated, which is much larger than the equilibration time for this system. 


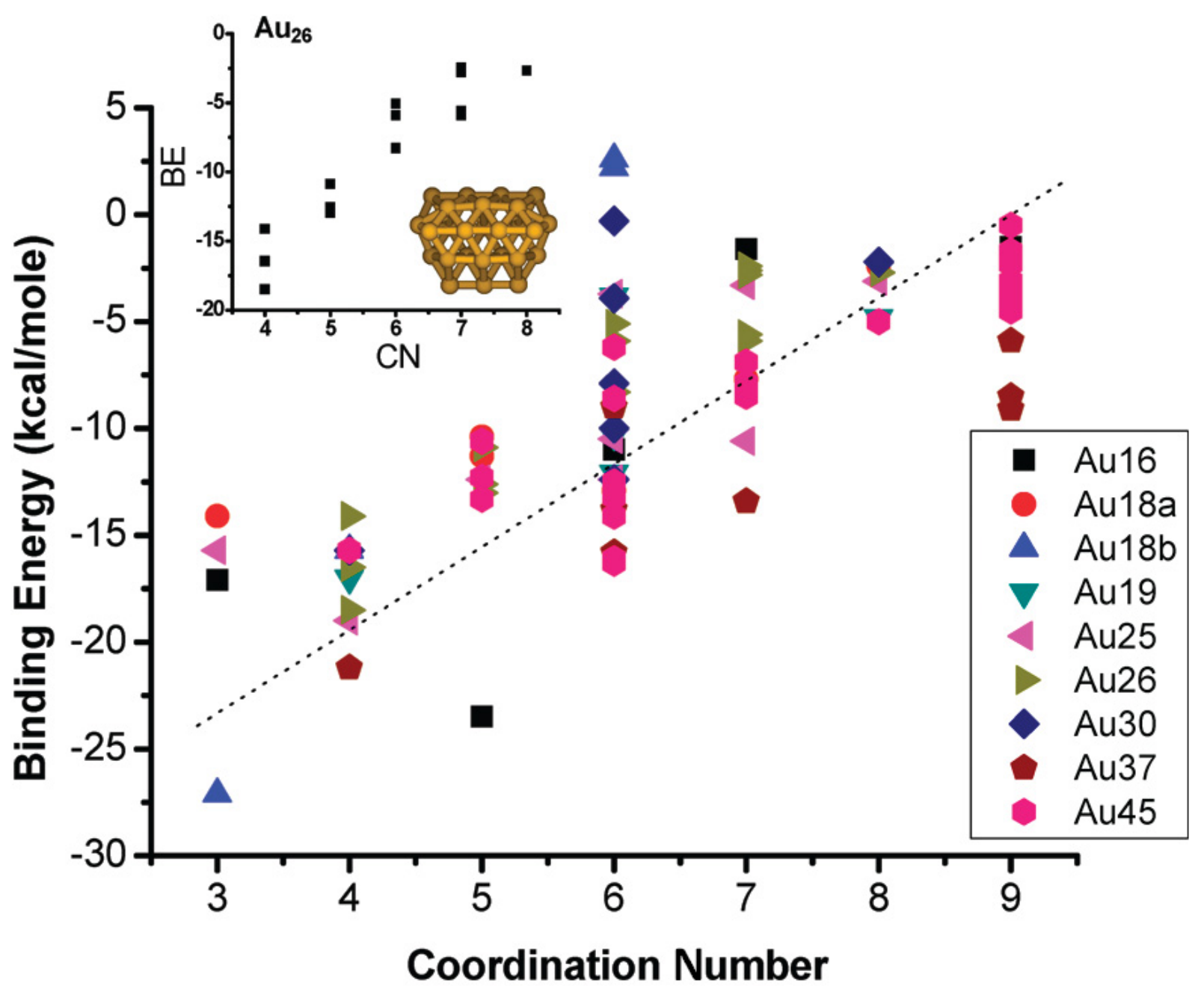

Figure 33. $\mathrm{CO}$ binding energy, Q, on different coordinated $\mathrm{Au}$ atoms of $\mathrm{Au}_{\mathrm{n}}$ clusters consisting of $\mathrm{n}$ atoms; $\mathrm{n}=16$, $18,19,25,26,30,37$, and 45 vs. Au atom coordination number $(\mathrm{CN})$. The CO binding energy (absolute value) decreases as the $\mathrm{Au} \mathrm{CN}$ increases (the dotted line is a guide to the eye). The large vertical scatter indicates that coordination number cannot be the only descriptor. Inset: Nanoparticle DFT calculations of binding energy for CO binding on $\mathrm{Au}$ (with 45 atoms) vs. the coordination number. A linear dependence of Q on $\mathrm{CN}$ is found. Taken from (Mpourmpakis et al., 2010a). 


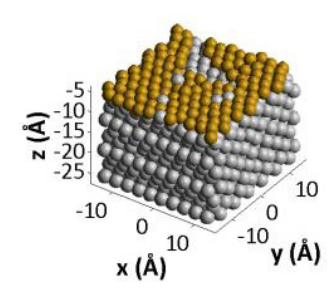

Molecular

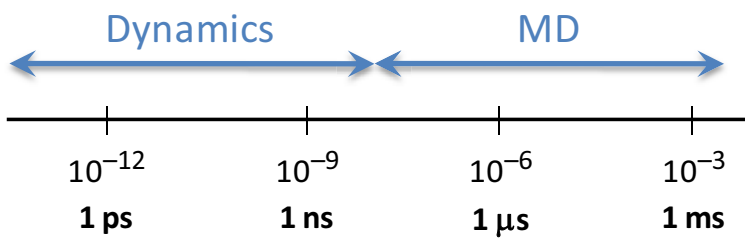

Nudged Elastic Band ( $\left.E_{\text {barrier }}\right)$
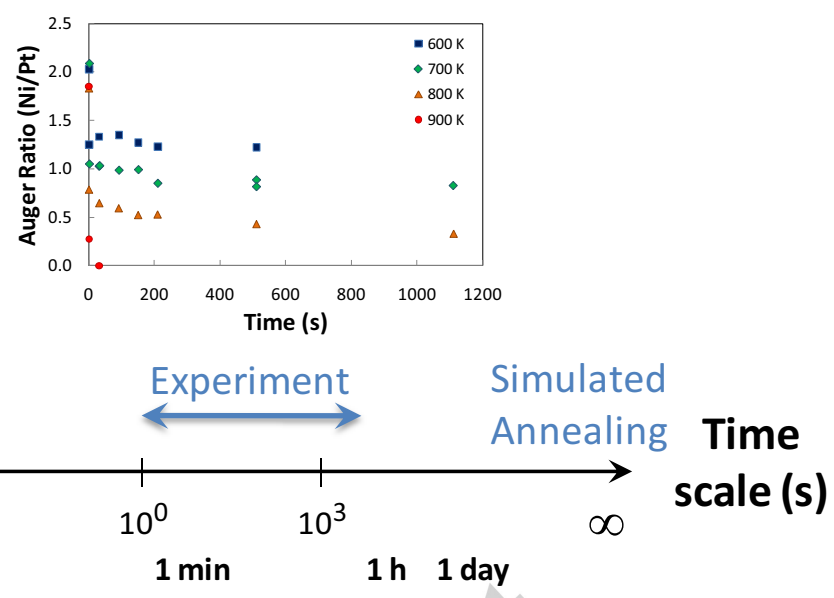

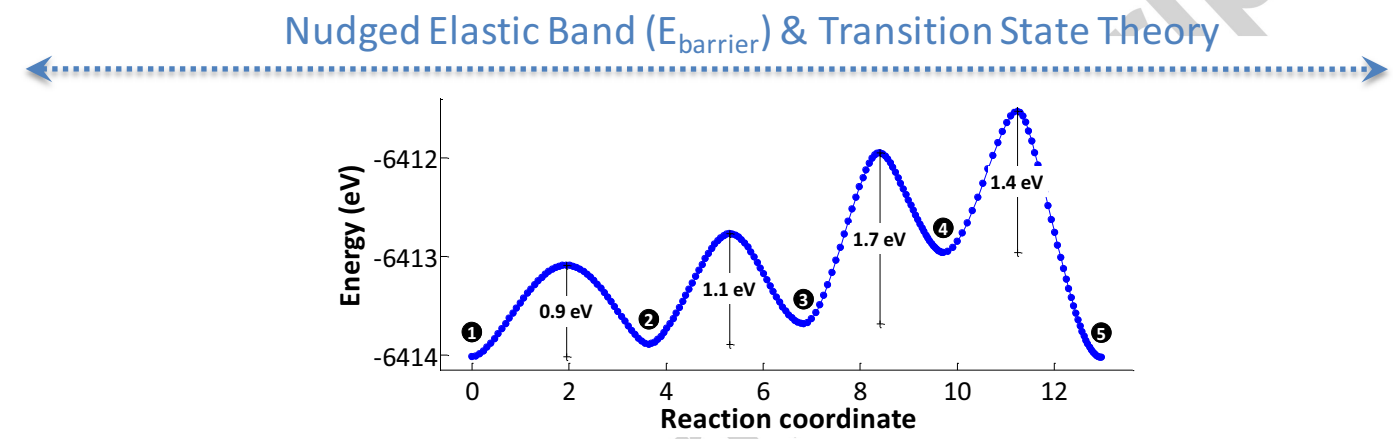

Figure 34. Multiscale modeling of $\mathrm{Ni} / \mathrm{Pt}$ mixing and experimental data. At the finer time-scale (order of several nanoseconds) molecular dynamics (MD) simulations were performed. Accelerated MD gave insights on the behavior of the system at longer time-scales (on the order of milliseconds). The infinite time behavior was investigated through simulated annealing. Nudge elastic band calculations provide a static picture of the potential energy surface; combined with transition state theory, they can provide insights on the timescale of mixing. Experimentally accessible time scales are on the order of several minutes. Based on simulation and experimental methodology presented in (Wang et al., 2010). 


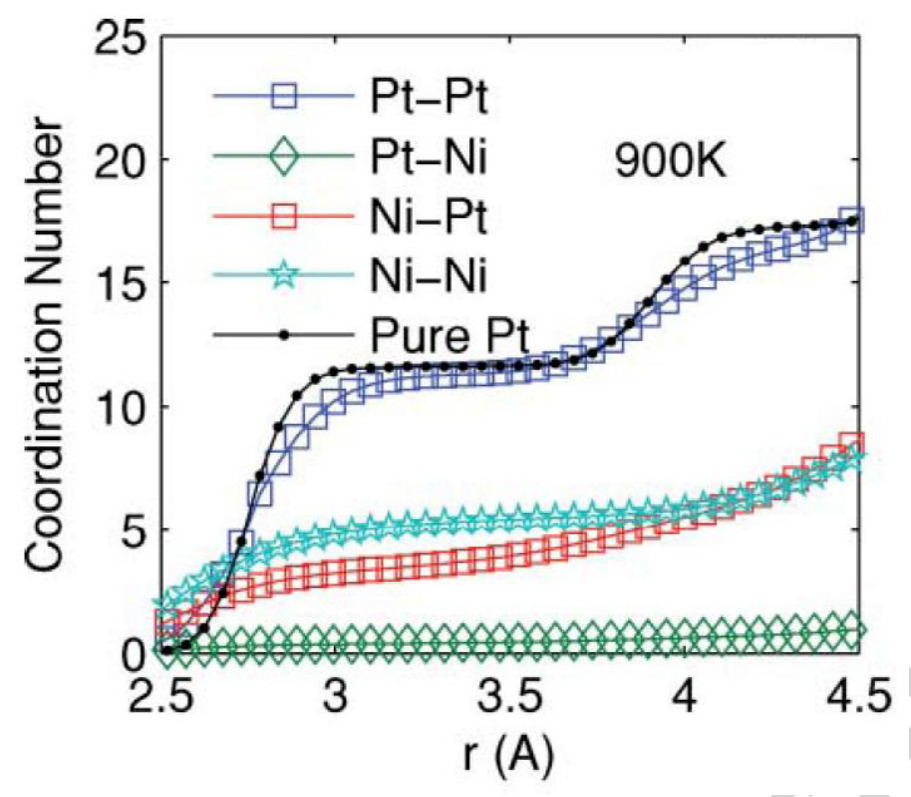

Figure 35. Coordination numbers $\mathrm{Pt}-\mathrm{Pt}, \mathrm{Pt}-\mathrm{Ni}$, Ni-Pt and $\mathrm{Ni}-\mathrm{Ni}$ in the $\mathrm{Ni} / \mathrm{Pt}(111)$ system at $900 \mathrm{~K}$, averaged from MD trajectories during the final $2 \mathrm{~ns}$ after the structure has equilibrated. The coordination number of $\mathrm{Pt}-\mathrm{Pt}$ in pure $\mathrm{Pt}$ bulk is also shown for comparison. $\mathrm{r}$ is the distance to the center atom. Taken from (Wang et al., 2010). 

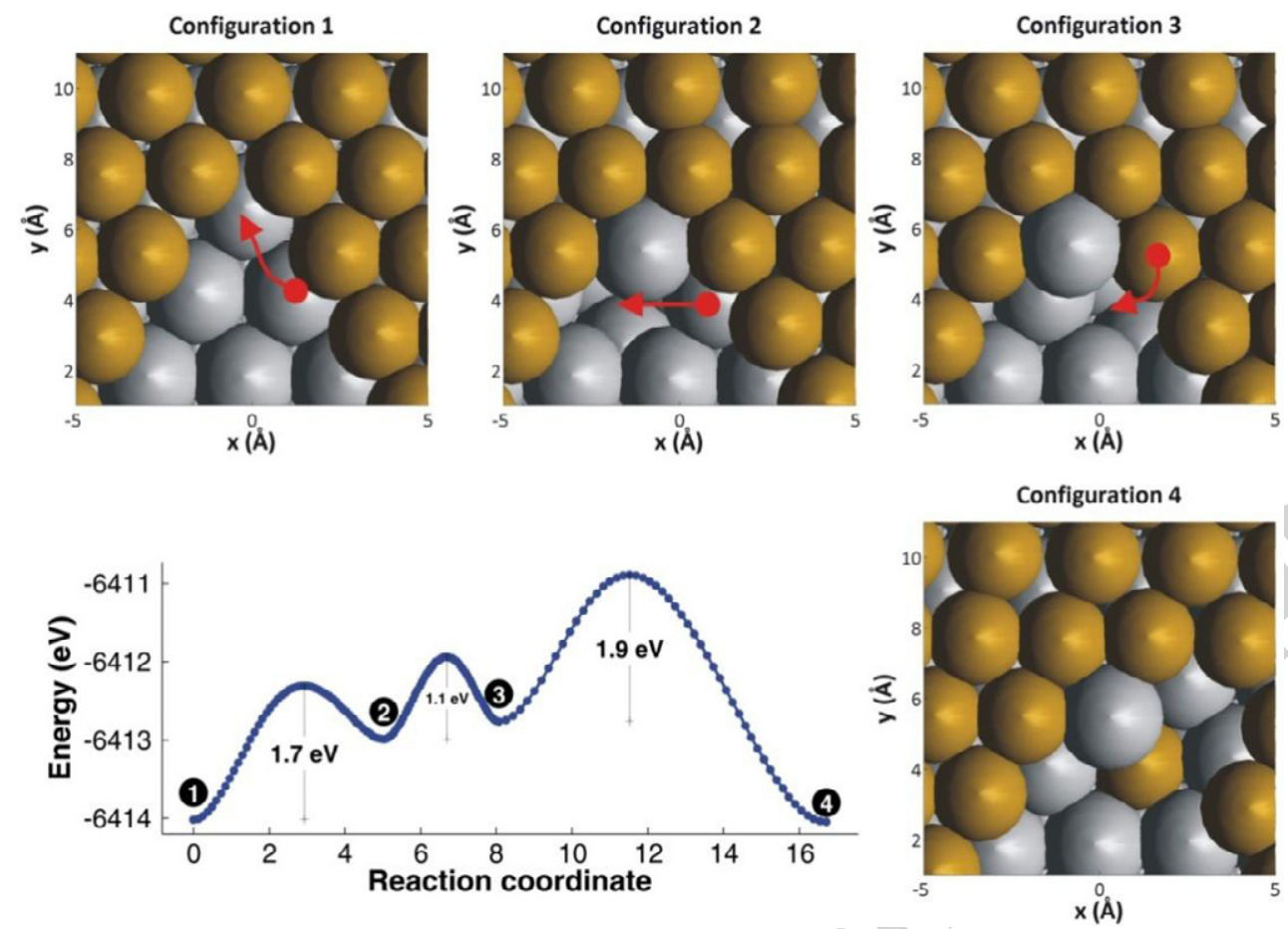

Figure 36. Results from nudge elastic band calculations in terms of potential energy surface and snapshots along it: a Pt atom close to the left edge of the "hole" enters the surface layer. Subsequently, a vacancy is formed in the first Pt layer, which diffuses to the right when a Pt atom hops in the opposite direction overcoming a barrier of $1.1 \mathrm{eV}$ (transition between configurations 2 and 3). Finally, a Ni atom from the surface monolayer fills this vacancy. Taken from (Wang et al., 2010). 


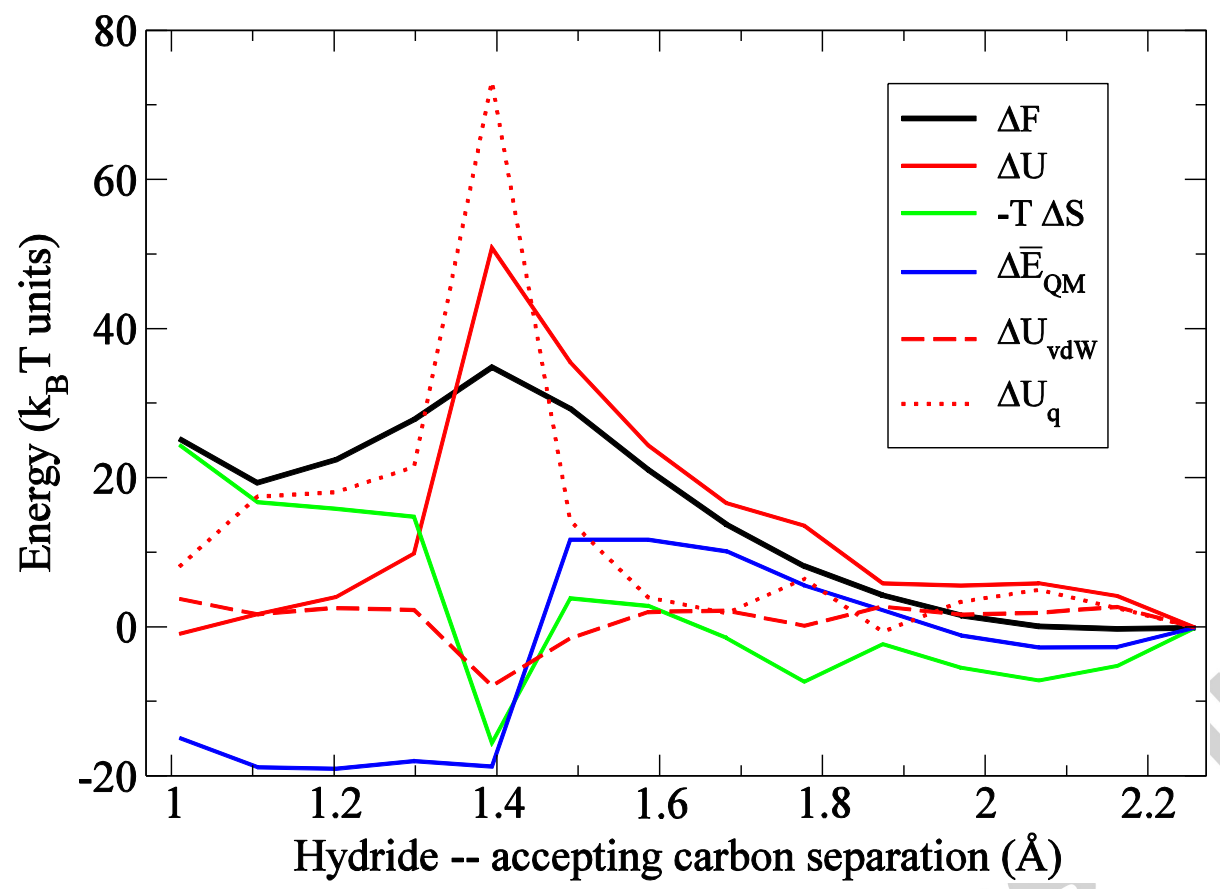

Figure 37. Energy profiles for hydride transfer during the multi-step dehydration of D-fructose to HMF in water. The free energy change, , is analysed in internal energy, , and entropy, , terms. The energy , is further analysed in contributions from the quantum mechanical energy of the reacting system, , the van der Waals interactions , and the electrostatic interactions . Taken from (Caratzoulas and Vlachos, 2011). 


\section{Research highlights}

A perspective on multiscale modeling of mechanism development

Estimation of thermochemistry and kinetics via hierarchical multiscale methods

Computation-driven catalyst design and discovery of materials with emergent behavior

Uncertainty on catalyst design, particle effects, and structure sensitivity of reactions

Free energies in solution and solvent effects in catalytic reactions 